UNIVERSIDADE DE SÃO PAULO

FACULDADE DE ADMINISTRAÇÃO, ECONOMIA E CONTABILIDADE DEPARTAMENTO DE ECONOMIA

PROGRAMA DE PÓS-GRADUAÇÃO EM ECONOMIA

Impacto de saltos no comportamento de preços de commodities

Paulo Martins Barbosa Fortes Manoel

Orientador: Prof. Dr. Denisard Cneio de Oliveira Alves

JANEIRO 2013

São Paulo 
Prof. Dr. João Grandino Rodas

Reitor da Universidade de São Paulo

Prof. Dr. Reinaldo Guerreiro

Diretor da Faculdade de Economia, Administração e Contabilidade

Prof. ${ }^{a}$ Dra. Elizabeth Maria Mercier Querido Farina

Chefe do Departamento de Economia

Prof. Dr. Pedro Garcia Duarte

Coordenador do Programa de Pós-Graduação em Economia 


\section{Impacto de saltos no comportamento de preços de commodities}

Dissertação apresentada ao Departamento de Economia da Faculdade de Economia, Administração e Contabilidade da Universidade de São Paulo como requisito para a obtenção do título de Mestre em Ciências.

Orientador: Prof. Dr. Denisard Cneio de Oliveira Alves

JANEIRO 2013

Versão Corrigida

(versão original disponível na Faculdade de Economia, Administração e Contabilidade) 
Manoel, Paulo Martins Barbosa Fortes

Impacto de saltos no comportamento de preços de commodities / Paulo Martins Barbosa Fortes Manoel - São Paulo, 2012.

$105 \mathrm{p}$.

Dissertação (Mestrado) - Universidade de São Paulo, 2011

Orientador: Denisard Cneio de Oliveira Alves.

1. Finanças 2. Derivativos 3. Petróleo

4. Métodos MCMC 5. Bolsa de mercadorias

I. Universidade de São Paulo. Faculdade de Economia, Administração

e Contabilidade. II. Título.

CDD - 332 
Aos meus pais, Sergio e Laura 


\section{AGRADECIMENTOS}

A entrega deste trabalho encerra um período que foi, de diversas maneiras, o mais frutífero da minha vida. No mestrado do IPE-USP vivi situações que promoveram meu amadurecimento acadêmico e pessoal: adquiri profundo conhecimento da teoria econômica com verdadeiros professores e fiz amigos que, com toda certeza, levarei para toda a vida. Nada mais justo, portanto, que agradecer todos aqueles que, de alguma maneira, me auxiliaram nesta caminhada.

Começo agradecendo minha mãe, Laura. Sem seu apoio e sua ênfase nos estudos esta dissertação jamáis seria consumada. Agradeço também meus irmãos, Daniel e André, pela paciência nos tempos de elaboração deste trabalho, e meus avós, Marta e Claro, por todo suporte e amor incondicional.

Agradeço meu colega e grande amigo Eduardo Ros pela paciência na leitura deste trabalho e por suas valiosas sugestões. Agradeço também meu orientador, Ricardo Avelino, pela sugestão do tema e pelos ensinamentos acerca da escrita de um trabalho científico.

Registro aqui minha gratidão e minha admiração aos meus companheiros de todos os eventos, dos profanos aos eruditos, das noites na D-Edge às óperas no Theatro Municipal, Andre Zilio, Victor Westrupp e Andre Mazini, grandes amigos que lograram tornar a passagem pelo mestrado prazerosa.

At last, but not at least, agradeço aos amigos que fiz neste mestrado. Nossa união foi de tal modo intensa que formamos um grupo especial, carinhosamente chamado de PARTIDINHO COPEIRO, composto pelos gaúchos bairristas André Zilio e Eduardo Ros; pelo sempre criativo Heleno; pelo arqueiro Marião; pelo dinamarquês Victor; pelo felino João; e pelo Cegonha, Eduardo. O mestrado termina aqui, mas nossa amizade permanece. 


\section{RESUMO}

Neste trabalho analisa-se a relevância de saltos no apreçamento de derivativos de commodities através da comparação de dois modelos. O primeiro leva em consideração um convenience yield com reversão à média, enquanto o segundo é uma generalização do primeiro com saltos no preço à vista. Ambos os modelos são estimados por meio de uma abordagem Bayesiana, sendo as distribuições a posteriori simuladas com o uso de técnincas da família MCMC. Dados de petróleo, trigo e cobre são utilizados para fins de estimação. A análise econométrica indica significância estatística para saltos, mas não encontrou-se evidência significativa de que saltos melhoram o apreçamento de derivativos. 


\begin{abstract}
In this work we analyze the relevance of jumps in the pricing of commodity contingent claims by comparing two models. The first takes into account mean-reverting convenience yields, and the second is a generalization of the first with jumps in spot prices. Both models were estimated using a Bayesian approach, and posterior distributions where simulated using MCMC techniques. Oil, copper and wheat data where used for estimation proposes. Econometric analysis indicates statistical significance for jumps, but we found no strong evidence that jumps improve derivative pricing.
\end{abstract}




\section{Sumario}

Lista de Tabelas $\quad$ xiii

Lista de Figuras $\quad$ XV

1. Introdução . . . . . . . . . . . . . . . . . . . . . . 1

2. Modelos de apreçamento . . . . . . . . . . . . . . . . . 7

$2.1 \quad$ Modelo CYE . . . . . . . . . . . . . . . . . . 7

2..1.1 Equações de Estado . . . . . . . . . . . . . 7

2..1.2 Medida neutra ao risco . . . . . . . . . . . . 9

$2 . .2 \quad$ Modelo CYES . . . . . . . . . . . . . . . . . . . . . 11

2..2.1 Equação de Estados . . . . . . . . . . . . . . . . . . 11

2.2.2 Medida neutra ao risco . . . . . . . . . . . . . . 12

2..3 Apreçamento de opções . . . . . . . . . . . . . . . . . . . . . . . . 14

3. Modelo econométrico . . . . . . . . . . . . . . . . . . . . 17

3.1 Método de simulação . . . . . . . . . . . . . . . . 21

4. Descrição dos dados . . . . . . . . . . . . . . . . . 25

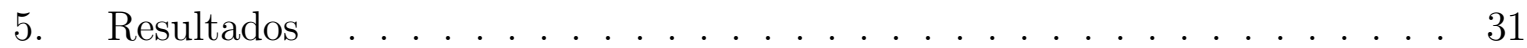

5..1 Parâmetros Estimados . . . . . . . . . . . . . . . . . 31

5..2 Comparação entre os modelos CYE e CYES . . . . . . . . . . . . 39

5..2.1 Comparação da REQM para Contratos Futuros Fora da Amostra . . . . . . . . . . . . . 39

$5 . .2 .2 \quad$ Normalidade dos Resíduos . . . . . . . . . . . . . . . 41

5..2.3 Comparação das REQM para Preços de Opções . . . . . . 44

6. Conclusão . . . . . . . . . . . . . . . . . . . . . . 49

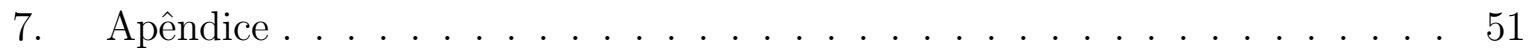

$7 . .1$ Apreçamento de contratos futuros . . . . . . . . . . . 51

$7 . .2 \quad$ Algoritmo LSM . . . . . . . . . . . . . . . . 57 
7..3 Prova de que a função de verossimilhança pode ser ilimitada na presença de saltos ... . . . . . . . . . . . . 6 61

$7 . .4$ Especificação Bayesiana . . . . . . . . . . . . . 63

7..5 Dedução das distribuições a posteriori . . . . . . . . . . . . . 65

7..5.1 Dedução de $\mu_{m} \mid \Theta_{-\mu_{m}}, \Delta q, \eta_{m}, \xi, \mathbf{y} \ldots \ldots . \ldots 65$

7..5.2 Dedução de $\alpha \mid \Theta_{-\alpha}, \Delta q, \eta_{m}, \xi, \mathbf{y} \ldots \ldots$. . . . . . 66

7..5.3 Dedução de $\hat{\alpha} \mid \Theta_{-\hat{\alpha}}, \Delta q, \eta_{m}, \xi, \mathbf{y} \ldots \ldots . . \ldots 67$

7..5.4 Dedução de $V^{-2} \mid \Theta_{-V}, \Delta q, \eta_{m}, \xi, \mathbf{y} \ldots \ldots . \ldots 68$

7..5.5 Dedução de $\bar{\eta}_{m} \mid \Theta_{-\bar{\eta}_{m}}, \Delta q, \eta_{m}, \xi, \mathbf{y} \ldots \ldots . . \ldots 69$

7..5.6 Dedução de $\omega^{-2} \mid \Theta_{\omega}, \Delta q, \eta_{m}, \xi, \mathbf{y} \ldots \ldots . \ldots 69$

7..5.7 Dedução de $\phi \mid \Theta_{\phi}, \Delta q, \eta_{m}, \xi, \mathbf{y} \ldots \ldots . . \ldots 70$

7..5.8 Dedução de $\Delta q_{i} \mid \Theta,\left\{\Delta q_{j}\right\}_{j \neq i}, \eta_{m}, \xi, \mathbf{y} \ldots \ldots \ldots$

7..5.9 Dedução de $\eta_{m i} \mid \Theta, \Delta q,\left\{\eta_{m j}\right\}_{j \neq i}, \xi, \mathbf{y} \ldots \ldots . . . . .71$

$7 . .6$ Descrição do algoritmo MCMC . . . . . . . . . . . . . . 73

7..6.1 Simulação de $\Sigma \ldots \ldots \ldots$. . . . . . . . . 73

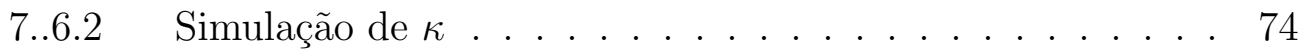

7..6.3 Simulação de $\left\{\xi_{i}\right\}_{i=i}^{N} \ldots \ldots \ldots . \ldots . \ldots . \ldots 75$

7..6.4 Algoritmo de MCMC completo . . . . . . . . . 77

7..7 Exemplo das cadeias de Markov: modelo CYES e dados de cobre . 79

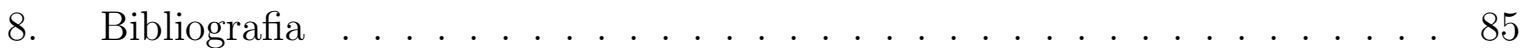




\section{Lista de Tabelas}

1 Valores teóricos para opções americanas e europeias no modelo CYES . . . 15

2 Estatísticas descritivas dos dados de petróleo . . . . . . . . . . . . 25

3 Estatísticas descritivas dos dados de trigo . . . . . . . . . . . . . . . . . 28

$4 \quad$ Estatísticas descritivas dos dados de cobre . . . . . . . . . . . . . . . . . 29

5 Estimação dos modelos CYE e CYES para dados de petróleo . . . . . . . . 32

6 Estimação dos modelos CYE e CYES para dados de trigo . . . . . . . . . . 35

7 Estimação dos modelos CYE e CYES para dados de cobre . . . . . . . . 36

8 Comparação entre erros de previsão dos modelos CYES e CYE estimados com os dados de petróleo do painel A . . . . . . . . . . . . . . . 39

9 Comparação entre erros de previsão dos modelos CYES e CYE estimados com os dados de petróleo trigo . . . . . . . . . . . . . . . . . . . . . . . . 40

10 Comparação entre erros de previsão dos modelos CYES e CYE estimados com os dados de cobre . . . . . . . . . . . . . . . . . . . . . . . . 40

11 Medidas de normalidade para os resíduos da equação do preço à vista . . . 43

12 Medidas de normalidade para os resíduos da equação do convenience yield 43

13 Comparação entre erros de previsão dos modelos CYES e CYE para dados de opções de futuros de petróleo . . . . . . . . . . . . . . . . . . 45

14 Especificação das distribuições a posteriori nos modelos CYE e CYES . . . 64 


\section{Lista de Figuras}

1 Maturidades históricas dos contratos futuros de petróleo do painel A . . . 26

2 Número médio de negociações do contrato F11 em um dia . . . . . . . . . 27

3 Número de negociações do contrato F7 em um dia . . . . . . . . . . . . . 28

4 Médias e intervalos de confiança para as variáveis de estado no modelo CYES utilizando os dados de petróleo do painel B . . . . . . . . . . . . . . 33

5 Estimativas da probabilidade e da magnitude dos saltos para dados do painel B de petróleo . . . . . . . . . . . . . . . . . . . . . . . 34

6 Médias e intervalos de confiança para as variáveis de estado no modelo CYES utilizando os dados de cobre . . . . . . . . . . . . . . . . . 36

$7 \quad$ Estimativas da probabilidade e da magnitude dos saltos para dados de trigo 38

8 QQ plot para os resíduos das equações de estado para dados de cobre . . . 41

9 QQ plot para os resíduos das equações de estado para dados de petróleo do painel A . . . . . . . . . . . . . . . . . . . . . . . 42

10 Evoulção histórica da amostra de opções americanas de petróleo e do con-

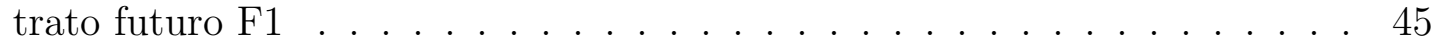

11 Dados de mercado e previsões para o contrato CLF1C 95.00 . . . . . . . 46

12 Simulação de $\mu_{m} \mid \mathbf{y}$ utilizando o modelo CYES e dados de cobre . . . . . . 79

13 Simulação de $\alpha \mid \mathbf{y}$ utilizando o modelo CYES e dados de cobre . . . . . . . 80

14 Simulação de $\kappa \mid \mathbf{y}$ utilizando o modelo CYES e dados de cobre . . . . . . . 80

15 Simulação de $\sigma_{S} \mid \mathbf{y}$ utilizando o modelo CYES e dados de cobre . . . . . . . 81

16 Simulação de $\sigma_{\delta} \mid \mathbf{y}$ utilizando o modelo CYES e dados de cobre . . . . . . . 81

17 Simulação de $\rho \mid \mathbf{y}$ utilizando o modelo CYES e dados de cobre . . . . . . . 82

18 Simulação de $\hat{\alpha} \mid \mathbf{y}$ utilizando o modelo CYES e dados de cobre . . . . . . . 82

19 Simulação de $V \mid \mathbf{y}$ utilizando o modelo CYES e dados de cobre . . . . . . . 83

20 Simulação de $\phi \mid \mathbf{y}$ utilizando o modelo CYES e dados de cobre . . . . . . . 83

21 Simulação de $\bar{\eta}_{m} \mid \mathbf{y}$ utilizando o modelo CYES e dados de cobre . . . . . . 84

22 Simulação de $\omega \mid \mathbf{y}$ utilizando o modelo CYES e dados de cobre . . . . . . . 84 


\section{Introdução}

O objetivo deste trabalho é responder a seguinte questão: são os saltos relevantes para o apreçamento de derivativos de commodities? Esta pergunta é motivada por uma série de artigos que encontram evidência empírica a respeito da existência de prêmio de risco de saltos nos preços de opções de ações, como Gurdip Bakashi \& Chen (1997), Bates (1991) e Pan (2002). Nenhum estudo a este respeito, no entanto, foi efetuado para o mercado de commodities, que goza de características distintas àquelas dos mercados de ações e requer uma análise específica.

A principal particularidade do mercado de commodities não compartilhada por outros mercados, como o de ações ou o de juros, é que a sua posse física pode representar um benefício ou um custo para o detentor. Os mecanismos que determinam estes custos e estes benefícios, assim como seus impactos nos preços futuros, são contemplados pela chamada teoria do armazenamento de Working (1949). Esta teoria sustenta que, quando o nível total de estoque de determinada commodity é alto, armazéns disponiveis são mais escassos, o que implica em um custo de armazenamento mais elevado; nesta situação os preços futuros devem ser, por não arbitragem, mais elevados que o preço à vista multiplicado pela taxa de juros livre de risco, um fenômeno conhecido como contango. Por outro lado, se o nível de estoques da commodity é baixo, armazéns livres seriam mais abundantes e, consequentemente, o custo de carrego estaria abaixo do fluxo de serviços providenciado pelo armazém ${ }^{1}$ e do potencial benefício que a posse física da commodity traz como a possibilidade de tomar vantagem de flutuações locais de preços e de demandas locais por produtos processados; neste caso os preços futuros devem situar-se abaixo do preço à vista multiplicado pela taxa de juros livre de risco, num fenômeno conhecido como backwardation.

\footnotetext{
${ }^{1} \mathrm{O}$ fluxo de serviços providenciado por um armazém pode compreender processamento, transporte e publicidade, entre outros. Uma análise mais detalhada destes serviços pode ser encontrada em Working (1949)
} 
O benefício líquido da posse física de uma commodity não usufruído pelo detentor de um contrato futuro é, portanto, um conceito chave na explicação da estrutura a termo de preços de commodities, recebendo o nome de convenience yield. Esta variável possui papel central em estudos recentes acerca do mercado de commodities. Black (1976) a assume constante e calcula fórmulas fechadas para preços de contratos futuros e de opções europeias. Gibson \& Schwartz (1990) mostra que um modelo de dois fatores, com um processo de reversão à média para o convenience yield, pode explicar os preços de contratos futuros com razoável precisão. Schwartz (1997), por fim, generaliza o modelo anterior com a inclusão de taxas de juros estocásticas com reversão à média.

Nos modelos acima citados, preços seguem uma trajetória continua. Ao verificar a relevância de saltos no apreçamento de commodities este trabalho também analisa se esta hipótese é válida ou impõe restrições excessivas à dinâmica estocástica dos preços de commodities, gerando insumos para pesquisas futuras.

A significância de saltos no apreçamento de derivativos é testada através da estimação de um modelo de preços de commodities que contempla um convenience yield que reverte à uma média de longo prazo e preços à vista com saltos. Este modelo foi introduzido por Hilliard \& Reis (1998) em uma abordagem teórica, onde são deduzidas fórmulas fechadas para preços de contratos futuros e de opções europeias, mas sem uma tentativa de estimação de seus parâmetros. É também estimado o modelo análogo sem saltos, como em Gibson \& Schwartz (1990), para em seguida comparar a aderência de ambos os modelos aos preços de derivativos observados no mercado.

A estimação de modelos com saltos envolve certas dificuldades técnicas e deve ser efetuada com precaução. Usualmente, parâmetros de modelos de apreçamento são estimados por máxima verossimilhança. Este método, no entando, perde sua validade no contexto de precessos com saltos. Honoré (1998) mostra que, no processo de saltos de Merton (1976), a função de verossimilhança é ilimitada, de modo que o estimador de máxima verossimilhança sequer está definido. Honoré (1998) propõe um estimador consistente 
para os parâmetros do modelo de Merton (1976), dependendo de algumas especificações do modelo. Uma possível solução mais geral, adotada neste trabalho, é o uso de uma distribuição a priori para os parâmetros, numa abordagem de estimação Bayesiana. Tal distribuição é capaz de eliminar as singularidades da função de verossimilhança que ocorrem na fronteira do espaço paramétrico onde a variância dos termos de erro aproximam-se de zero. Neste ponto, o problema de estimação se reduz ao problema computacional do cálculo de momentos da distribuição de probabilidade a posteriori.

Um ferramental indicado para lidar com cálculos envolvendo distribuições de probabilidade de altas dimensões (como é o caso da distribuição a posteriori deste trabalho) na literatura econométrica é o da classe de algoritmos das Cadeias de Markov via Monte Carlo (MCMC segundo a abreviação em inglês). Esta família provê métodos gerais e elegantes para simular uma determinada distribuição de probabilidade através da construção de uma cadeia de Markov com propriedades de convergência desejáveis. A ideia básica consiste em armazenar uma cadeia suficientemente grande de valores correlacionados que satisfazem a uma lei dos grandes números apropriada, de modo que momentos da distribuição original podem ser aproximados com arbitrária precisão.

A construção da citada cadeia de Markov envolve a seleção de uma densidade geradora de candidatos para cada parâmetro (ou para cada bloco de parâmetros, dependendo de como o algoritmo for desenhado), sendo o algoritmo final composto por uma sequencia de passos de amostragens para cada (bloco de) parâmetro(s). Para uma densidade geradora de candidatos genérica o passo do algoritmo recebe o nome de Metropolis-Hastings. Se, no entanto, formos capazes de gerar amostras da verdadeira distribuição marginal do parâmetro (ou bloco de parâmetros) em questão, o passo do algoritmo recebe o nome de Gibbs-Sampling, um caso particular dos algoritmos de Metropolis-Hastings. Métodos de Gibbs-Sampling são desejáveis em relação a métodos genéricos de Metropolis-Hastings pois, em geral, logram gerar cadeias de menor auto-correlação. Sua viabilidade prática depende, no entanto, da possibilidade de gerar amostras das distribuições marginais, o que nem sempre é possível. 
A variável a ser simulada neste trabalho é a distribuição conjunta dos saltos, dos parâmetros e das variáveis latentes (o preço à vista e o conveniene yield) condicionados aos valores das variáveis de medida, os contratos futuros negociados nas bolsas de futuros. Utilizou-se um algoritmo de simulação da família MCMC que consiste de uma mistura de passsos do tipo Metropolis-Hastings genérico e de passos do tipo Gibbs-Sampling, sempre adotando o último quando possível. As variáveis latentes foram simuladas por meio do método de Jong \& Shephard (1995), que permite a amostragem de um série de variáveis de estado que satisfazem as hipóteses do filtro de Kalman clássico.

Os momentos da distribuição a posteriori foram calculados utilizando-se dados de petróleo, cobre e trigo. Econtrou-se significância estatística para saltos nos dados de petróleo e trigo. Ademais, identifiou-se um salto percentual médio signficantemente positivo para os dados de trigo, em contraste com a literatura de saltos em preços de ações que, via de regra, encontra saltos médios negativos.

Visando mensurar o ganho que a inclusão do termo de salto representa em relação ao modelo sem saltos, compara-se neste trabalho suas performances em termos de apreçamento de derivativos fora da amostra de estimação. Esta análise foi efetuada utilizando-se (1) contratos futuros com maturidades fora do intervalo de estimação e (2) opões americanas em futuros de petróleo. A análise da raíz quadrada do erro quadrático médio (REQM) mostra que o modelo com saltos apresenta uma pequena, porém estatisticamente insignificante, vantagem no apreçamento de contratos futuros. A comparação dos erros de previsão dos preços de opções americanas também não mostra uma melhor aderência do modelo com saltos, indicando que, no caso de commodities, saltos não apresentam a mesma relevância no apreçamento de derivativos que aquela verificada no mercado de ações.

Este trabalho está organizado da seguinte maneira. Os modelos teóricos de apreçamento de commodities, em suas versões com e sem saltos, assim como os resultados básicos acerca do apreçamento de derivativos utilizados neste trabalho, encontram-se na seção 2 . O modelo econométrico e o método de simulação da distribuição a posteriori são estudados 
na seção 3. A descrição das bases de dados de petróleo, cobre e trigo é feita ne seção 4. Os resultados da análise econométrica e a comparação dos modelos com saltos e sem saltos em termos de apreçamentos de derivativos e de normalidade dos resíduos econtram-se na seção 5 . 


\section{Modelos de apreçamento}

Nesta são estudados, de um ponto de vista estritamete teórico, dois modelos. O primeira leva em consideração um convenience yield com reversão à uma média de longo prazo e foi intrduzido por Gibson \& Schwartz (1990). O segundo é uma generalização do primeiro incorporando saltos no preço à vista e foi introduzido por Hilliard \& Reis (1998). Ambos os modelos são de simples manipulação e possuem fórmulas fechadas para preços de contratos futuros.

Ao longo deste trabalho, o primeiro modelo será denominado modelo de convenience yield estocástico (CYE). Já o segundo modelo será denominado modelo de convenience yield estocástico com saltos (CYES). Ambos os modelos supõe que (1) transações ocorrem continuamente no tempo; (2) não existem custos de transação, taxas ou restrições para posições vendidas; e (3) a taxa de juros livre de risco é uma constante $r$.

\section{2..1 Modelo CYE}

\section{2..1.1 Equações de Estado}

Este modelo possui duas variáveis de estado: o preço à vista da commodity, S, e o convenience yield instantâneo, $\delta$, que atua como um fluxo de serviços ao detentor da commodity. Supõe-se que a lei de movimento destas variáveis é dada pelas seguintes equações de difusão:

$$
\begin{gathered}
d S=(\mu-\delta) S d t+\sigma_{S} S d Z_{S} \\
d \delta=\kappa(\alpha-\delta) d t+\sigma_{\delta} d Z_{\delta}
\end{gathered}
$$

onde (1) $\mu$ é o retorno de longo prazo ${ }^{1}$ da commodity; (2) $\alpha$ é a média de longo prazo

\footnotetext{
${ }^{1} \mathrm{O}$ retorno de longo prazo da commodity será a soma do fluxo de serviços $\delta$ com a variação esperada para o preço, $\mu-\delta$.
} 
do convenience yield; (3) $\kappa$ é a velocidade de ajustamento do convenience yield à sua média de longo prazo; (4) $\sigma_{S}$ e $\sigma_{\delta}$ são os devios padrão dos termos de erro das equações do preço à vista e do convenience yield, respectivamente; e (5) $d Z_{S}$ e $d Z_{\delta}$ são movimentos Brownianos correlacionados por $\rho_{S \delta}$.

O retorno de longo prazo $\mu$ deve, idealmente, estar acima da taxa de juros livre de risco $r$, pois um fato estilizado a respeito do mercado de commodities é que seus preços tendem a acompanhar o ciclo econômico.

A média de longo prazo para o convenience yield, $\alpha$, encontra uma interpretação na teoria do armazenamento: quanto maior seu valor, maior é o benefício líquido médio em mantê-la estocada e menores são os custos médios de armazenamento.

Para poder capturar certos fatos estilizados acerca do mercado de commodities, onde situações de contango e backwardation ocorrem de maneira alternada no tempo, devemos permitir que $\delta$ oscile em torno de sua média $\alpha$. A velocidade com que $\delta$ reverte à sua média é dada pelo parâmetro $\kappa$; isto é, após um choque $\sigma_{\delta} d Z_{\delta}, \delta$ retornará para sua média $\alpha$ mais rapidamente para valores maiores de $\kappa^{1}$.

O parâmetro de correlação entre os termos de erro do preço e do convenience yield, $\rho_{S \delta}$, é esperado positivo pela teoria do armazenamento, pois ambos os choques resultam de movimentos no nível total de estoques da commodity. Numa situação de aumento do nível de estoques o preço à vista deve, para manter o equilíbrio entre oferta e demanda, diminuir; simultaneamente, armazéns disponiveis devem tornar-se mais escassos, o que implicará em um aumento do custo de estocagem e em uma diminuição do convenience yield. O raciocínio no caso de uma diminuição do nível total de estoques é análogo e leva a um aumento tanto do preço à vista quanto do convenience yield.

\footnotetext{
${ }^{1}$ Com efeito, a meia-vida deste processo, isto é, o tempo médio necessário para que $\delta$ atinja o ponto médio entre $\delta$ e $\alpha$, será inversamente proporcional a $\kappa$.
} 


\section{2..1.2 Medida neutra ao risco}

Esta subseção trata de uma breve discussão da medida neutra ao risco e do apreçamento dos contratos futuros no modelo CYE, necessária para o objetivo dste trabalho de comparar o apreçamento de drivativos entre os modelos com saltos e sem saltos. Uma análise pormenorizada pode ser encontrada em Bjerksund (1991) ou no apêndice 7..1.

Utilizando o Lemma de Ito e definindo $X=\log (S)$ a equação do preço à vista toma a seguinte forma:

$$
d X=\left(\mu-\delta-\frac{1}{2} \sigma_{S}^{2}\right) d t+\sigma_{S} d Z_{S}
$$

Neste modelo, o convenience yield instantâneo $\delta$ funciona da mesma maneira que um fluxo de dividendos. Consequentemente, o termo de tendência da equação do preço à vista na medida neutra ao risco toma a forma $r-\delta$, onde $r$ é a taxa de juros livre de risco, suposta constante. Logo, o processo para as variáveis de estado na medida neutra ao risco toma a forma

$$
\begin{gathered}
d X^{*}=(r-\delta) d t+\sigma_{S} d Z_{S}^{*} \\
d \delta^{*}=\left[\kappa(\alpha-\delta)-\lambda_{\delta}\right] d t+\sigma_{\delta} d Z_{\delta}^{*}
\end{gathered}
$$

onde $\lambda_{\delta}$ é o preço do risco do convenience yield ${ }^{1}$. Neste trabalho seguiremos a hipótese de Schwartz (1997) de que $\lambda_{\delta}$ é constante. Este pressuposto é feito com o intuito de manter o modelo o mais simples possível e de não tornar o processo de estimação excessivamente complexo; é perfeitamente razoável, no entanto, que $\lambda_{\delta}$ esteja relacionado com as variáveis de estado ou com o ciclo econômico, e possiveís parametrizações de $\lambda_{\delta}$ podem motivar pesquisas futuras.

Seja $F(T, S, \delta)$ o preço de um contrato futuro distando $T$ anos da maturidade. Pode-se

\footnotetext{
${ }^{1} \mathrm{O}$ parâmetro $\lambda_{\delta}$ pode ser intepretado como o custo de securitização do risco de variação do convenience yield e deve ser, idealmente, positivo.
} 
mostrar que, escolhendo uma combinação adequada deste contrato futuro e da commodity subjacente, obtém-se um portfolio livre de risco. Por não arbitragem, este portfolio deverá ter como retorno a taxa de juros livre de risco. Igualando estes retornos obtém-se a seguinte equação de derivadas parciais:

$$
\frac{1}{2} F_{S S} \sigma_{S}^{2} S^{2}+\frac{1}{2} F_{\delta \delta} \sigma_{\delta}^{2}+F_{S \delta} S \sigma_{S} \sigma_{\delta} \rho_{S \delta}+F_{S} S(r-\delta)+F_{\delta}\left[(\alpha-\delta)-\lambda_{\delta}\right]-F_{t}=0
$$

Bjerksund (1991) resolve esta equação analiticamente, obtendo a seguinte fórmula fechada para os preços futuros:

$$
\log (F(T))=X-\delta \frac{1-e^{-\kappa T}}{\kappa}+A(T)
$$

onde $A(T)$ não depende das variáveis de estado e seu valor pode ser encontrado no apêndice $7 . .1$ (fórmula 7.70$)$.

Esta relação linear entre as variáveis de estado e os preços futuros tem consequencias relevantes para a estrutura a termo de preços de commodities. Note, primeiramente, que mudanças no preço à vista implicam em deslocamentos paralelos da curva de log-preços futuros. Logo, qualquer tipo de mudança na inclinação da curva de log-preços deve estar, necessariamente, atrelada a uma variação do convenience yield instantâneo. Outro fato interessante é que, quanto maior a maturidade do contrato, ou quanto maior a velocidade de reversão à média $\kappa$, menor é o impacto do convenience yield no preço futuro. Isso decorre do fato de que, nestas situações, o valor do convenience yield instantâneo provê pouca informação a respeito da sua trajetória futura.

A equação 2.7 mostra ainda que o modelo ECY contempla situações de backwardation e

de contango. Com efeito, como $\frac{1-e^{-\kappa T}}{\kappa}>0$, preços futuros serão maiores (menores) que o preço à vista multiplicado pela taxa de juros para $\delta$ positivo (negativo) suficientemente grande em módulo, caracterizando contango (beckwardation). 


\section{2..2 Modelo CYES}

\section{2..2.1 Equação de Estados}

Este modelo generaliza o modelo CYE de uma maneira natural para contemplar saltos no preço à vista como em Merton (1976), Bates (1991) e Hilliard \& Reis (1998). Além do convenience yield estocástico e do preço à vista, duas novas variáveis de estado são incorporadas ao modelo: o número de saltos realizados, $q$, seguindo um processo de Poisson, e o valor (percentual) do salto, $\eta$, seguindo uma distribuição log-normal. Uma maneira de pensar este modelo é que, em quase todos os instantes de tempo, o preço à vista e o convenience yield seguem o processo de difusão do modelo CYE. Quando os saltos são acionados, sempre em instantes discretos, o preço à vista se move de maneira descontinua com incremento $\Delta S=\eta S$.

Mais precisamente, além das hipóteses de taxa de juros constante, de negociações realizadas continuamente no tempo e de ausência de custos de transação ou de taxas, supõe-se que as variáveis de estado $(S, \delta, q, \eta)$ seguem as equações 2.2 e

$$
\frac{d S}{S}=(\mu-\delta-\phi \bar{\eta}) d t+\sigma_{S} d Z_{S}+\eta d q
$$

onde (1) $\mu$ é o retorno de longo prazo da commodity; (2) $q$ é um processo de Poisson com parâmetro de intensidade (anualizado) $\phi ;(3) \log (1+\eta)$ segue distribuição normal com média $\log (1+\bar{\eta})-0.5 \omega^{2}$ e desvio padrão $\omega$.

O parâmetro de intensidade de Poisson, $\phi$, representa a quantidade esperada de saltos em um ano e, caso saltos sejam eventos de baixa frequencia, não deve possuir grande magnitude.

O parâmetro $\bar{\eta}$, como já foi dito, representa o choque percentual médio nos preços quando o salto é acionado. Não há uma expectativa a priori para seu sinal, podendo inclusive ser nulo no caso de saltos simétricos. 


\subsubsection{Medida neutra ao risco}

Como Merton (1976) observou, neste modelo não é possível construir uma carteira livre de risco composta pela commodity e por uma cesta de derivativos com as hipóteses feitas até o momento: em qualquer carteira deste gênero restaria um risco de salto não securitizável. Para proceder com o apreçamento de derivativos por não arbitragem, fundamental para a comparação entre os modelos CYE e CYES, serão necessários pressupostos adicionais.

Na literatura de saltos há dois possíveis conjuntos de hipóteses que permitem a dedução da medida neutra ao risco e o consequente cálculo de preços de derivativos. Ambos serão enunciados nesta subseção.

O primeiro trabalho a obter fórmulas de derivativos para este tipo de processo foi Merton (1976). Este trabalho contorna o problema dos saltos não securitizáveis supondo que (1) o CAPM é uma descrição válida para o equilíbrio do mercado de ativos e (2) o risco do saltos não é sistêmico. A hipótese (2) é, neste trabalho, justificada no caso de ações de uma única companhia pelo argumento de que saltos seriam causados por novas informações disponibilizadas e, sendo este um efeito firma-específico, não influenciaria o mercado como um todo. Logo, no arcabouço do CAPM, tal risco poderia ser eliminado sem custos.

O mérito destas hipóteses é sua simplicidade, mas carecem de realismo no caso do mercado commodities. Nota-se que tal mercado é composto por grandes grupos de matérias-primas e, sendo cada um destes grupos de elevado valor financeiro total, não parece razoável considerá-los partes desprezíveis da carteira de mercado.

Hipóteses alternativas são feitas por Bates (1991) para estimar um modelo de saltos para o índice S\&P 500, num contexto em que a existência de risco de saltos não sistêmico é extremamente inverossímil. Suas hipóteses, que serão adotadas neste trabalho, referemse ao lado da demanda por ativos, sendo: (1) os agentes poupadores são homogêneos; (2) a renda otimamente investida por estes agentes segue um processo de difusão com saltos simultâneos aos saltos do preço do ativo estudado; e (3) a utilidade do agente representativo é separavel no tempo, com aversão ao risco constante. 
Com estas hipóteses Bates (1991) deduz a equação de movimento para o preço à vista na medida neutra ao risco. A adaptação do seu argumento para o modelo de commodities, feita por Hilliard \& Reis (1998), é trivial e corresponde à seguinte equação:

$$
\frac{d S^{*}}{S^{*}}=\left(r-\delta-\phi^{*} \bar{\eta}^{*}\right) d t+\sigma_{S} d Z_{S}+\eta^{*} d q^{*}
$$

onde, na medida neutra ao risco, $d q^{*}$ é um processo de Poisson com parâmetro de intendidade $\phi^{*}$ e $\bar{\eta}^{*}$ segue uma distribuição log-normal de média $\bar{\eta}^{*}$.

A respeito dos parâmetros de salto na medida neutra ao risco, Bates (1991) observa que

"the true parameters (...) do not differ qualitatively or quantitatively from risk-neutral parameters"

Suporemos, então, que $\phi^{*}=\phi$ e que $\bar{\eta}^{*}=\bar{\eta}$. Utilizamos este pressuposto porque, como será visto, o processo de estimação deste trabalho não permite a estimação dos parâmetros de saltos na medida neutra ao risco. Eles serão, no entanto, utilizados no apreçamento de opções no modelo CYES. Note que, utilizando estas hipóteses, chegamos aos mesmos resultados que aqueles obtidos com os pressupostos de Merton (1976).

O cálculo do preço de contratos futuros é extremamente simples e segue o argumento de Hilliard \& Reis (1998). Sejam $\tilde{S}^{*}$ e $S^{*}$ as trajetórias (estocásticas) do preço à vista na medida neutra ao risco nos modelos CYE e CYES, respectivamente. Como vale que $\mathbf{E}^{*}\left(\eta^{*} d q^{*}\right)=\phi \bar{\eta}$ (onde $\mathbf{E}^{*}$ representa o operador esperança na medida neutra ao risco) deve-se ter, necessariamente, que $\mathbf{E}^{*}\left(S_{T}^{*}\right)=\mathbf{E}^{*}\left(\tilde{S}_{T}^{*}\right)$, uma igualdade entre os preços de contratos futuros com maturidade em $T$ em ambos os modelos.

Logo, o preço de contratos futuros no modelo CYES é dado pela equação 2.7. Isto se deve ao fato de que satos alteram a curtose e a assimetria dos preços no futuro segundo a medida neutra ao risco, mas não mudam sua média. O apreçamento de opções, no entanto, apresenta certas mudanças, como será discutido da próxima seção. 


\section{2..3 Apreçamento de opções}

Uma das grandes dificuldades relacionadas ao uso de opções para comparar os modelos CYE e CYES é o de que, em geral, a liquidez do mercado de opções de commodities concetra-se em opções americanas, cujo apreçamento é ainda um desafio para a teoria de derivativos financeiros.

Diferentemente das opções europeias, que tem uma data fixa para seu exercício, opções americanas podem ser exercidas durante todo o intervalo de tempo até seu vencimento. Isto implica que seu apreçamento deve levar em conta a distribuição de probabilidade do preço em todos os instantes de tempo até a sua maturidade, e não apenas a distribuição da data do vencinto, como no caso de opções europeias. Tal apreçamento é, portanto, extremamente mais complexo e raramente logra obter fórmulas analíticas fechadas.

Usualmente, preços de opções americanas são calculados por meio de métodos numéricos. Os métodos mais utilizados, como o de diferenças finitas e o da árvore binomial, não são adequados para este trabalho pois, como observa Longstaff \& Schwartz (2001),

"(...) finite difference and binomial techniques become impractical in situations where there are multiple factors."

Para fins de cálculo de preços de opções de compra americanas, será utilizado neste trabalho o chamado método de mínimos quadrados de Monte Carlo (LSM, segundo a sigla em inglês) de Longstaff \& Schwartz (2001). Este método consiste dos seguintes passos: (1) simulação de $Z$ trajetórias das variáveis de estado segundo a medida neutra ao risco; (2) estimação recursiva do valor de não exercer a opção em cada instante por meio de mínimos quadrados ordinários; (3) cálculo do preço da opção em cada instante e em cada trajetora como o máximo entre o valor de não exercício e o valor de exercício; e (4) cálculo do preço atual como a média (entre todas as $Z$ trajetórias) dos preços no momento imediatamente seguinte, descontados pela taxa de juros livre de risco. 
Para fins de ilustração, exibe-se na tabela 1 os valores teóricos para preços de opções europeias (segundo a fórmula fechada de Hilliard \& Reis (1998)) e americanas (calculadas pelo método LSM) de um contrato futuro da commodity para diferentes configurações dos parâmetros e das variáveis de estado.

\begin{tabular}{|ccc|cc|cc|cc|cc|}
\hline \multicolumn{3}{|c|}{ Parâmetros } & \multicolumn{2}{c|}{$S=35, \delta=-0.1$} & \multicolumn{2}{|c|}{$S=45, \delta=-0.05$} & \multicolumn{2}{c|}{$S=35, \delta=0.05$} & \multicolumn{2}{c|}{$S=45, \delta=0.1$} \\
\hline$\phi$ & $\bar{\eta}$ & $\sigma_{\delta}$ & Euro. & Amer. & Euro. & Amer. & Euro. & Amer. & Euro. & Amer. \\
\hline 1 & -0.20 & 0.05 & 3.77 & 5.50 & 9.37 & 13.18 & 2.87 & 4.38 & 7.63 & 10.65 \\
1 & -0.20 & 0.30 & 3.91 & 5.50 & 9.51 & 12.94 & 3.01 & 4.25 & 7.78 & 10.65 \\
1 & 0.20 & 0.05 & 3.71 & 5.41 & 9.08 & 12.75 & 2.87 & 4.29 & 7.39 & 10.58 \\
1 & 0.20 & 0.30 & 3.85 & 5.55 & 9.23 & 12.96 & 3.00 & 4.24 & 7.55 & 10.54 \\
5 & -0.20 & 0.05 & 6.52 & 7.68 & 12.41 & 14.63 & 5.44 & 6.40 & 10.67 & 12.51 \\
5 & -0.20 & 0.30 & 6.61 & 7.67 & 12.50 & 14.93 & 5.53 & 6.49 & 10.76 & 12.48 \\
5 & 0.20 & 0.05 & 6.16 & 7.67 & 11.67 & 16.65 & 5.18 & 6.73 & 10.02 & 12.58 \\
5 & 0.20 & 0.30 & 6.25 & 7.76 & 11.78 & 14.61 & 5.26 & 6.39 & 10.13 & 12.53 \\
\hline
\end{tabular}

Tabela 1: Valores teóricos para opções americanas e europeias no modelo CYES

Exibe-se nesta tabela os preços de opções de compra americanas e europeias para diferentes configurações de parametros e de variáveis de estado no modelo CYES. Preços de opções americanas são calculados com a fórmula fechada de Hilliard \& Reis (1998), enquanto preços de opções americanas são calculados utilizando o método LSM com base nos seguintes parâmetros (para uma explicação sobre estes parâmetros, checar apêndice 7..2): 100,000 trajetórias simuladas, divisão do intervalo de exercício em 100 pontos e funções básicas consistindo de todos os polinomios de Laguerre (ponderados) até ordem três. Utilizou-se também uma opção com maturidade de 0.75 anos de um contrato futuro da commodity com maturidade de um ano, $\sigma_{S}$ de $0.3, \kappa$ de $2, \rho$ de 0.2 , $\hat{\alpha}$ de $0.1, \omega$ de $0.05, r$ de 0.06 , e um preço strike para o contrato futuro de 40 .

Nota-se que o preço da opção é sensível a variações do parâmetro $\phi$. Na tabela exibese configurações com alta frequencia de salto $(\phi=5)$ e com baixa frequencia de salto $(\phi=1)$. Situações de alta frequecia de saltos tendem, ceteris paribus, a gerar preços mais elevados para as opções, contemplando assim o chamado prêmio de risco de salto. Tome, por exemplo, uma variação de $\phi$ fixando $\bar{\eta}$ em -0.20 e $\sigma_{S}$ em 0.05. Quando $\phi$ muda seu valor de 1 para 5 o preço da opção americana salta de 3.77 para 6.52 , uma variação de, aproximadamente, $73 \%$.

Outro fato interessante que pode ser destacado destas simulações é que a média do valor percentual do salto, $\bar{\eta}$, afeta o preço de opções de maneira assimétrica; isto é, valores de $\bar{\eta}$ de mesmo módulo mas de sinais distintos não afetam os preços das opções do mesmo modo, sendo que, ceteris paribus, valores negativos tendem a gerar preços mais elevados para opções. Isto ocorre porque, como observado em Bates (1991), a assimetria dos preços 
futuros na medida neutra ao risco tem o mesmo sinal de $\bar{\eta}$, sendo mais curvados para a direita no caso $\bar{\eta}>0$.

O exemplo acima mostra que o modelo com saltos gera, em teoria, deferenças quantitativas e qualitativas em relação ao modelo sem saltos. Isto não significa, no entanto, que o modelo CYES irá, a priori, ter mais aderência aos dados de mercado. Esta questão só pode ser respondida após a estimação dos modelos CYE e CYES e o posterior cotejo dos seus erros de previsão. Esta estimação é precedida por uma discussão teórica do método econométrico utilizado na intepretação dos dados, que é feita na próxima seção. 


\section{Modelo econométrico}

Esta seção é destinada a uma discussão teórica do método de estimação utilizado para estimar os modelos CYE e CYES. Fazemos também uma breve descrição dos métodos usuais de estimação de modelos de apreçamento e explicamos porque estes métodos não são apropriados para estimar o modelo CYES.

O primeiro passo do processo de estimação é a definição do conjunto informacional disponível ao econometrista. Este é um ponto crítico para modelos de apreçamento de commodities pois, como observado por Schwartz (1997),

"(...) one of the difficulties in the empirical implementation of commodity price models is that frequently the factors or state variables are not directly observable."

Preços à vista são negociados de modo descentralizado e sem uma padronização adequada do produto comercializado, o que dificulta a precisa definição de um preço à vista em um dado instante de tempo. O problema do convenience yield é ainda pior, pois se trata de uma abstração de potenciais ganhos especulativos que o investidor pode usufruir ${ }^{1}$. Contratos futuros, por outro lado, são negociados com grande transparencia, de modo centralizado e padronizado. Estas características desejáveis os tornam ideais para compor a amostra do modelo econométrico aqui abordado.

Neste trabalho será adotada a hipótese de Schwartz (1997) de que as únicas variáveis observadas pelo econometrista são os preços de contartos futuros negociados nas bolsas de futuros, sendo as variáveis de estado (isto é, o preço à vista e o convenience yield) não observáveis.

Descreve-se de maneira mais precisa, nas próximas linhas, o processo gerador de dados, assim como a parte destes dados que comporá o conjunto de informação observável. O

\footnotetext{
${ }^{1}$ Como, por exemplo, aproveitar flutuações locais de preços no local onde a commodity está armazenada.
} 
econometrista observa os dados de mercado apenas nos instantes $t_{1}, t_{2}, \ldots t_{N-1}, t_{N}$ com $t_{i+1}-t_{i}=\Delta t$ para todo $i$. Em cada instante $t_{i}$ a informação disponível será o vetor de logaritmos de preços de contratos futuros $\mathbf{y}_{i}=\left\{y_{i}^{j}\right\}_{1 \leq j \leq K}$ cujo vetor de anos à maturidade é constante no tempo e dado por $\mathbf{T}=\left(T_{1}, T_{2}, \ldots, T_{K}\right)^{\top}$.

Os log-preços futuros observados podem envolver spreads de bid-ask, não-simultaneidade das observações, limites de preço, erros de medida, etc. Supõe-se neste trabalho que todos esses ruídos podem ser representados por erros aditivos homocedásticos e não autocorrelacionados. Assim, a relação entre as variáveis de estado e o log-preço futuro pode, segundo a equação 2.7, ser sistematizada na seguinte equação de medida:

$$
\mathbf{y}_{i}=\mathbf{d}+\mathbf{Z} \xi_{i}+\mathbf{v}_{i}
$$

onde $\xi_{i}=\left(X_{i}, \delta_{i}\right)^{\prime}$ é o vetor de variáveis latentes, $\left\{\mathbf{v}_{i}\right\}_{1 \leq i \leq N}$ é um conjunto de variáveis multivariadas não-correlacionadas noramente distribuídas com matriz de variância $V^{2} \mathbf{I}_{K x K}$ e as matrizes $\mathbf{d}$ e $\mathbf{Z}$ são definidas por

$$
\begin{gathered}
\mathbf{d}=\left(\begin{array}{c}
A\left(T_{1}\right) \\
A\left(T_{2}\right) \\
\vdots \\
A\left(T_{K}\right)
\end{array}\right), \\
\mathbf{Z}=\left(\begin{array}{cc}
1 & -\frac{1-e^{-\kappa T_{1}}}{\kappa} \\
1 & -\frac{1-e^{-\kappa T_{2}}}{\kappa} \\
\vdots & \vdots \\
1 & -\frac{1-e^{-\kappa T_{K}}}{\kappa}
\end{array}\right) .
\end{gathered}
$$


Antes de prosseguir com a descrição do método de estimação via MCMC, o problema da não linearidade entre as variáveis de estado $\xi_{i}$ imposto pela lei de movimento 2.3 deve ser endereçado ${ }^{1}$. A relação analítica entre $\xi_{i+1}$ e $\xi_{i}$ é complexa e sua utilização no algoritmo de simulação de Metropolis-Hastings o tornaria indesejavelmente complicado.

A solução adotada para contornar este problema é a utilização da linearização (conhecida como aproximação de Euler) da equação de mudança de estados 2.3. Como observa Johannes \& Polson (2002), esta prática é usual no contexto de estimação de modelos de difusão via MCMC e, se o incremento temporal $\Delta t$ é sufucientemente pequeno, incorre-se em erros desprezíveis ${ }^{2}$.

A equação de mudança de estados para o modelo CYES, baseada na equação 2.3 e já em sua forma linearizada, toma a forma

$$
\xi_{i+1}=\mathbf{c}_{i}+\mathbf{Q} \xi_{i}+\varepsilon_{i}
$$

onde

$$
\begin{gathered}
\mathbf{c}_{i}=\left(\begin{array}{c}
\left(\mu-\phi \bar{\eta}-\frac{1}{2} \sigma_{S}^{2}\right) \Delta t+\eta_{i} \Delta q_{i} \\
\kappa \alpha \Delta t
\end{array}\right), \\
\mathbf{Q}=\left(\begin{array}{cc}
1 & -\Delta t \\
0 & 1-\kappa \Delta t
\end{array}\right) . \\
\Sigma \equiv \mathbf{E}\left(\varepsilon_{i} \varepsilon_{i}^{\top}\right)=\left(\begin{array}{cc}
\Delta t \sigma_{s}^{2} & \Delta t \rho_{S \delta} \sigma_{s} \sigma_{\delta} \\
\Delta t \rho_{S \delta} \sigma_{s} \sigma_{\delta} & \Delta t \sigma_{\delta}^{2}
\end{array}\right) . \\
P\left(\Delta q_{i}=1\right)=\phi \Delta t, P\left(\Delta q_{i}=0\right)=1-\phi \Delta t .
\end{gathered}
$$

\footnotetext{
${ }^{1}$ Note que a lei de movimento das variáveis de estado 2.3 é linear apenas para variações infinitesmais de tempo, mas não para variações discretas.

${ }^{2}$ Neste trabalho utiliza-se $\Delta t=\frac{7}{365} \approx 0.02$ para dados semanais, mesmo valor utilizado por Schwartz (1997).
} 
A representação em espaço de estados dada pelas equações 3.10 e 3.13 pode ser utilizada para o cálculo da função de verossimilhança dos parâmetros condicionados aos valores das variáveis de medida por meio do filtro de Kalman. A abordagem natural, a partir deste ponto, seria a maximização desta função para a obtenção dos estimadores de máxima verossimilhança, como fez Schwartz (1997) para o modelo CYE. Este procedimento é, no entanto, inválido no contexto de processos com saltos. Isto porque, conforme provado em Honoré (1998), log-retornos em um modelo desta categoria são uma mistura de distribuições normais, e um resultado a respeito deste tipo de distribuição é o de que sua função de verossimilhança é ilimitada se os parâmetros de média e de variância são desconhecidos, tendendo ao infinito em certos pontos do espaço paramétrico em que a variância se aproxima de zero. Uma prova desta afirmação pode ser encontrada no apêndice 7..3.

Contorna-se este problema neste trabalho adotando-se uma distribuição a priori informativa para os parâmetros. A ideia básica é a de que uma distribuição a prori que atribui baixa densidade de probabilidade à região do espaço paramétrico em que a variância dos termos de erro aproxima-se de zero pode eliminar as citadas singularidades da função de verossimilhança. Isto pode ser feito, por exemplo, com a hipótese de uma distribuição $a$ priori gamma inversa para os parâmetros de variância, cuja densidade de probabilidade converge exponencialmente para zero quando seu argumento vai para zero.

Esta hipótese altera o objeto de estimação e de inferência no modelo econométrico. Em vez de obter estimativas de um parâmetro numérico fixo, no arcabouço da estatística clássica, este trabalho tratará do estudo da distribuição a posteriori ${ }^{1}$ dos parâmetros, isto é, da distribuição dos parâmetros condicionados às variáveis de medida observadas no mercado, numa abordagem de estimação Bayesiana. Para prosseguir com o processo de estimação, faz-se necessária a especificação do modelo Bayesiano adotado, isto é, a definição das variáveis a serem estimadas e suas distribuiçoes a priori. O vetor de parâmetros a ser estimado, $\Theta$, é uma simples reparametrização do modelo como apresentado na seção 2.,

\footnotetext{
${ }^{1}$ A distribuição a posteriori de um conjunto de parâmetros $\Theta$ condicionados aos valores de certa varável de medida y é dada pela regra de Bayes $p(\Theta \mid \mathbf{y}) \propto p(\mathbf{y}) p(\mathbf{y} \mid \Theta)$.
} 
adotada para tornar o método de simulação mais simples. Sua definição precisa, assim como sua distribuição a priori, podem ser encontrados no apêndice 7..4.

\subsection{Método de simulação}

Neste ponto, o problema de inferência reduz-se ao problema computacional do cáluclo de momentos da seguinte distribuição de probabilidade a posteriori:

$$
p\left(\Theta,\left\{\xi_{i}\right\}_{i=1}^{N},\left\{\Delta q_{i}\right\}_{i=1}^{N-1},\left\{\eta_{i}\right\}_{i=1}^{N-1} \mid \mathbf{y}\right)
$$

Em outras palavras, deseja-se estudar a distribuição de probabilidade conjunta dos parâmetros, dos preços à vista, do convenience yield e dos saltos condicionados à informação de mercado dos preços dos contratos futuros.

Uma técnica adequada para o estudo de uma distribuição de probabilidade de alta dimensão, como é o caso de 3.18, é o de simulação via Metropolis-Hastings. Este método tem como objetivo a amostragem de uma cadeia cuja medida estacionária coincide com a distribuição de probabilidade estudada.

Antes de iniciar a descrição do algoritmo de Metropolis-Hastings, convém, por simplicidade, fixar uma notação. Chamaremos a variável aleatória completa a ser simulada de $\Phi$, isto é,

$$
\Phi=\left\{\Theta,\left\{\xi_{i}\right\}_{i=1}^{N},\left\{\Delta q_{i}\right\}_{i=1}^{N-1},\left\{\eta_{i}\right\}_{i=1}^{N-1}\right\} \mid \mathbf{y}
$$

O primeiro passo para a aplicação do algoritmo de Metropolis-Hastings é a definição dos blocos de parâmetros que serão simulados simultaneamente ${ }^{1}$. Considere uma divisão de $\Phi$ em $n$ blocos disjuntos, isto é, $\Phi=\Phi_{1} \cup \Phi_{2} \cup \cdots \cup \Phi_{n}$ e $\Phi_{i} \cap \Phi_{j}=\emptyset$ se $i \neq j$ (uma descrição precisa dos blocos adotados neste trabalho pode ser encontrada no apêndice 7..6).

\footnotetext{
${ }^{1}$ Segundo Chib (2001), tais blocos devem conter varaveis altamente correlacionadas, a fim de que a convergencia da cadeia seja mais veloz
} 
O passo seguinte é o da escolha de uma densidade geradora de candidatos para cada bloco. A densidade associada ao bloco $i$, denotada por $h_{i}\left(\Phi_{i}^{c} \mid \Phi_{1}, \Phi_{2}, \ldots, \Phi_{n}\right)$, especifica uma medida de probabilidade para a amostragem de um candidato $\Phi_{i}^{c}$ para a cadeia de Markov a ser gerada. Este candidato, no entanto, pode não compor a cadeia. Ele será aceito com a seguinte probabilidade $\nu$ :

$$
\nu=\min \left\{\frac{p\left(\Phi_{i}^{c}, \Phi_{-i}\right) h_{i}\left(\Phi_{i} \mid \Phi_{i}^{c}, \Phi_{-i}\right)}{p\left(\Phi_{i}, \Phi_{-i}\right) h_{i}\left(\Phi_{i}^{c} \mid \Phi_{i}, \Phi_{-i}\right)}, 1\right\}
$$

onde $\Phi_{-i}$ representa o vetor de parâmetros excluindo-se o bloco $i$. A probabilidade de aceite tem como função "moldar" os valores gerados pela densidade $h_{i}$ para ficarem próximos da distribuição marginal de $\Phi_{i}$.

Suponha que estejamos aptos a simular perfeitamente a distribuição marginal do bloco $\Phi_{i}$. Neste caso, a densidade geradora de candidatos seria dada por

$$
h_{i}\left(\Phi_{i}^{c} \mid \Phi_{i}, \Phi_{-i}\right)=p\left(\Phi_{i}^{c} \mid \Phi_{-i}\right)
$$

e a probabilidade de aceite fica

$$
\nu=\min \left\{\frac{p\left(\Phi_{i}^{c}, \Phi_{-i}\right) p\left(\Phi_{i} \mid \Phi_{-i}\right)}{p\left(\Phi_{i}, \Phi_{-i}\right) p\left(\Phi_{i}^{c} \mid \Phi_{-i}\right)}, 1\right\}=1
$$

de modo que o candidato é sempre aceito. Esse caso é conhecido como Gibbs-Sampling e, em geral, é preferido em relação a métodos de Metropolis-Hastings genéricos, mas dependem da possibilidade de se gerar amostras das verdadeiras distribuições marginais, o que nem sempre é possível. No apêndice 7..6, que contém uma descrição mais precisa do algoritmo MCMC utilizado neste trabalho, pode-se encontrar a descrição dos blocos em que se logrou a utilização do método de Gibbs-Sampling.

O último passo para a aplicação do algoritmo é a definição do número $M$ de elementos que serão armazenados na cadeia de Markov. Valores maiores de $M$ tornam a cadeia gerada mais próxima da sua medida estacionária, mas são limitados pela memória computacional disponível. No presente trabalho utiliza-se $M=10,000$. 
O algoritmo completo de Metropolis-Hastings pode então ser enunciado da seguinte maneira:

1. Especifique um chute inicial $\Phi^{(0)}=\left(\Phi_{1}^{(0)}, \Phi_{2}^{(0)}, \ldots, \Phi_{n}^{(0)}\right)$

2. Faça, para $m=1, \ldots, M$,

(a) Faça, para $i=1, \ldots, n$,

i. Gere um candidato $\Phi_{i}^{c} \sim h_{i}\left(\Phi_{i}^{c} \mid \Phi_{1}^{(m)}, \ldots, \Phi_{i-1}^{(m)}, \Phi_{i}^{(m-1)}, \ldots, \Phi_{n}^{(m-1)}\right)$

ii. calcule

$$
\nu=\min \left\{\frac{p\left(\Phi_{i}^{c}, \Phi_{-i}\right) h_{i}\left(\Phi_{i}^{(m-1)} \mid \Phi_{i}^{c}, \Phi_{-i}\right)}{p\left(\Phi_{i}^{(m-1)}, \Phi_{-i}\right) h_{i}\left(\Phi_{i}^{c} \mid \Phi_{i}, \Phi_{-i}\right)}, 1\right\}
$$

onde $\Phi_{-i}=\left(\Phi_{1}^{(m)}, \ldots, \Phi_{i-1}^{(m)}, \Phi_{i}^{(m-1)}, \ldots, \Phi_{n}^{(m-1)}\right)$

iii. defina

$$
\Phi_{i}^{(m)}= \begin{cases}\Phi_{i}^{c} & \text { se } \nu>\text { Uniforme }(0,1) \\ \Phi_{i}^{(m-1)} & \text { caso contrario }\end{cases}
$$

3. Retorne $\left\{\Phi^{(1)}, \Phi^{(2)}, \ldots, \Phi^{(M)}\right\}$.

Em geral, para tentar minimizar a influencia do chute inicial $\Phi^{(0)}$ no resultado final, descarta-se um trecho inicial da cadeia antes de se começar a gravar os $M$ valores, o chamado burn-in. Neste trabalho utiliza-se um burn-in de 2,000.

Os resultados básicos de convergencia da cadeia $\left\{\Phi^{(1)}, \Phi^{(2)}, \ldots, \Phi^{(M)}\right\}$ encontram-se em Chib (2001). Para fins práticos, pode-se supor que $\left\{\Phi^{(1)}, \Phi^{(2)}, \ldots, \Phi^{(M)}\right\}$ segue a distribuição de $\Phi$ e que momentos de funções de $\Phi$ podem ser calculados utilizando-se leis dos grandes números e médias amostrais da cadeia gerada.

Uma descrição mais precisa do algoritmo de estimação pode ser encontrado no apêndice 7..6. No que segue, todas as estimativas de parâmetros são baseadas nas médias das distribuições a posteriori calculadas por meio desta simulação. 


\section{Descrição dos dados}

Os dados utilizados para fins de estimação consistem de observações semanais (em cada sexta-feira) de preços de contratos futuros de petróleo, cobre e trigo. Em cada estimação utilizou-se quatro maturidades distintas (isto é, $K=4$ )

A tabela 2 mostra as estatísticas descritivas básicas dos contratos futuros de petróleo da NYMEX utilizados nas estimações deste trabalho. Os dados de petróleo consistem de dois painéis: um primeiro, chamado de painel A, contemplando os contratos F1, F3, F7 e F10 no intervalo de tempo que vai de 6/1/1995 a 24/8/2012, e um segundo, chamado de painel B, contendo os contratos F1, F3, F7 e F10 no intervalo de 4/9/1987 a 24/8/2012. Aqui F1 denota o contrato futuro mais próximo da maturidade, F2 denota o segundo contrato mais próximo da maturidade e assim por diante.

\begin{tabular}{ccc}
\hline Contrato Futuro & $\begin{array}{c}\text { Preço Médio } \\
\text { (Desvio Padrão) }\end{array}$ & $\begin{array}{c}\text { Maturidade Média } \\
\text { (Desvio Padrão) }\end{array}$ \\
\hline \multicolumn{3}{c}{ Painel A: De 6/1/1995 a $24 / 8 / 2012$} \\
F1 & $47.88(30.10)$ & $0.072(0.024)$ \\
F5 & $48.21(31.03)$ & $0.405(0.024)$ \\
F10 & $48.03(31.62)$ & $0.822(0.024)$ \\
F15 & $47.76(31.83)$ & $1.239(0.024)$ \\
\hline \multicolumn{3}{c}{ Painel B: De 4/9/1987 a $24 / 8 / 2012$} \\
\hline F1 & $39.55(28.46)$ & $0.072(0.035)$ \\
F3 & $39.68(28.95)$ & $0.241(0.080)$ \\
F1 & $39.59(29.52)$ & $0.577(0.180)$ \\
\hline
\end{tabular}

Tabela 2: Estatísticas descritivas dos dados de petróleo

Esta tabela contém as médias e os desvios padrão para o preço e para os anos à maturidade de cada contrato utilizado para fins de estimação.

Como a data de maturidade de cada contrato é fixa, o número de anos à maturidade deve decrescer com o tempo. Sempre que F1 matura, no entanto, há uma rolagem dos contratos: F2 transforma-se em F1, F3 transforma-se em F2 e assim por diante, o que faz com que o número de anos à maturidade retorne ao antigo patamar, sempre dentro de uma pequena faixa de variação, como pode ser observado na figura 1. 


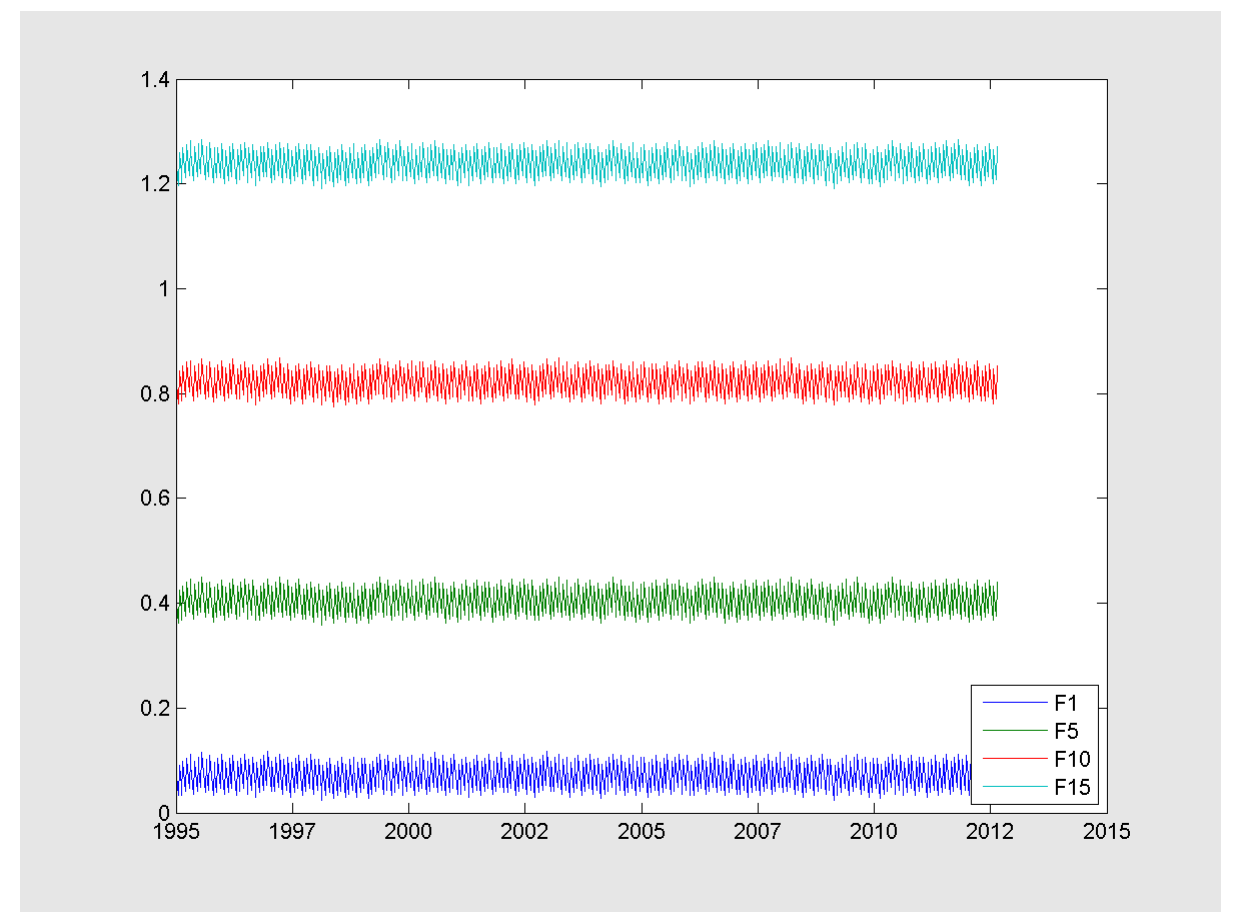

Figura 1: Maturidades históricas dos contratos futuros de petróleo do painel A Esta figura mostra, com uma frequencia semanal, as maturidades (em anos) de cada um dos quatro contratos utilizados na estimação deste trabalho, começando em 6/1/1995 e com término em 24/8/2012

Para fins de estimação, cada contrato será tratado como se seu número de anos à maturidade fosse constante, de modo que o modelo econométrico da seção 3 pode ser utilizado. Esta hipótese é motivada pelos valores muito baixos de desvio padrão da maturidade exibidos na tabela 2 , variando entre 0.02 e 0.2 no pior dos casos.

A utilização de dois painéis distintos para os dados de petróleo busca contemplar dois tipos de amostra: uma que possua contratos de maior maturidade, o painel A, e um que possua maior histórico, o painel B. Não é possível construir uma amostra com estas duas propriedades porque os contratos acima de F10 eram extremamente ilíquidos antes da década de 90. A figura 2, que exibe o número médio de negociações diarias do contrato F11 em uma janela móvel de doze meses, ilustra este fato. Nota-se que o número de negociações era irrisório antes da década de 90, começa a ganhar impulso em 1989 e adquire maior consistência após 1995, rompendo definitivamente a barreira dos 2,500 contratos diários e chegando a valores superiores a 20,000 contratos por dia em períodos recentes 


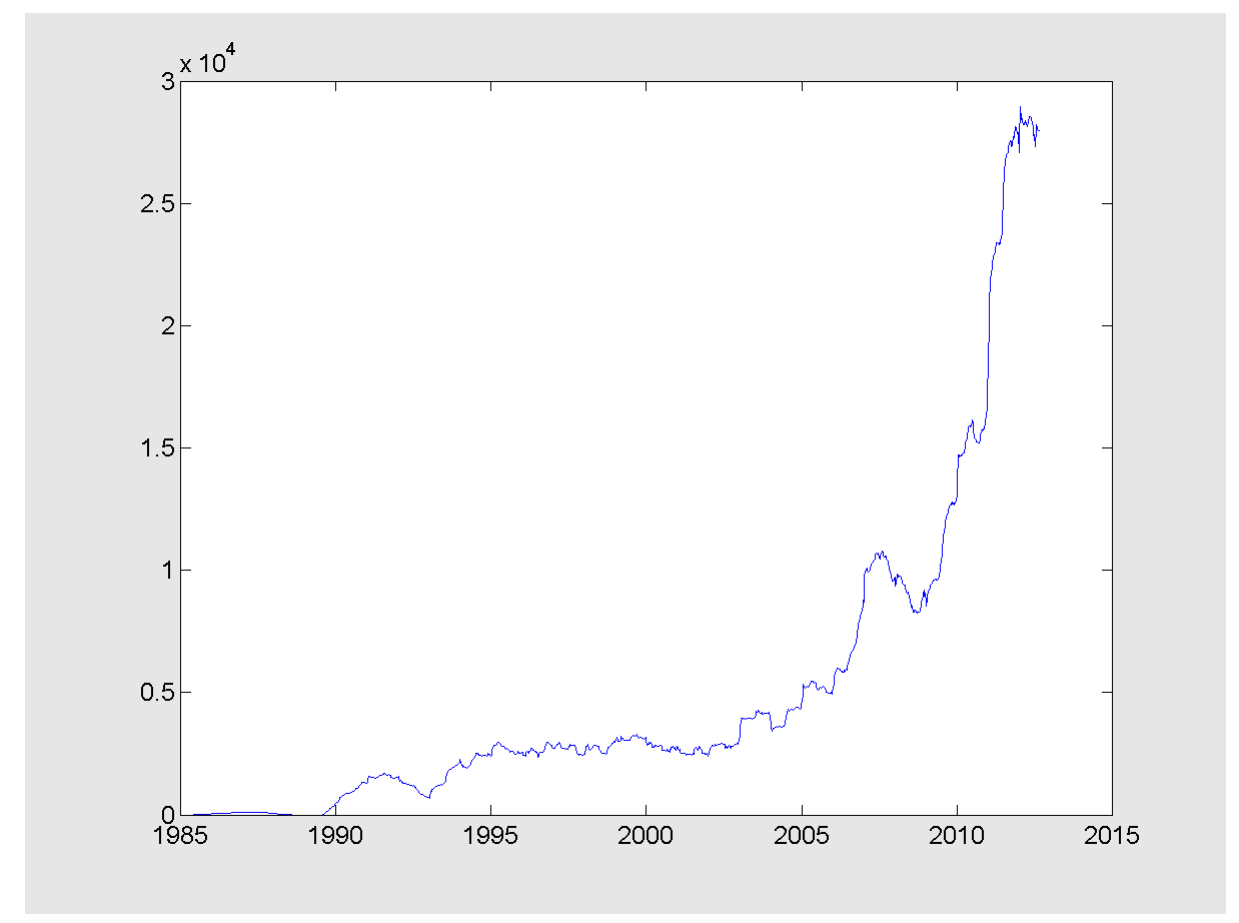

Figura 2: Número médio de negociações do contrato F11 em um dia

Esta figura mostra mostra o número médio de negociações diarias do contrato futuro de petróleo F11 numa janela móvel de doze meses.

Os dados de trigo da bolsa de Chicago, cujas estatísticas descritivas básicas estão reportadas na tabela 3, apresentam problema semelhante. Os contratos até F6 apresentam considerável volume de negociação após 1989, de aproximadamente 2,000 contratos por dia, em média. O contrato F7 (com maturidade de 1.3 anos), no entanto, apresenta liquidez apenas em certos períodos, chegando a não ser negociado em diversas datas, como pode ser visto no gráfico 3. Os contratos acima de F7 (F8, F9 até F15) apresentam, via de regra, pior liquidez em todas as datas.

Em face deste problema, optou-se por utilizar apenas os contratos F1, F3, F5 e F6 para os dados de trigo. A utilização do contrato F7 só poderia ser feita em datas após 2006, data em que este contrato atinge volumes ne negociação mais consistentes, o que tornaria a amostra indesejavelmente pequena.

Nota-se que em ambas as bases de dados abordadas até o momento (os painéis de petróleo e o painel de trigo), os contratos futuros mais longos têm aproximadamente um ano de maturidade. Contratos mais longos poderiam estimar os parâmetros da estrutura a termo 


\begin{tabular}{ccc} 
Contrato Futuro & $\begin{array}{c}\text { Preço Médio } \\
\text { (Desvio Padrão) }\end{array}$ & $\begin{array}{c}\text { Maturidade Média } \\
\text { (Desvio Padrão) }\end{array}$ \\
\hline \multicolumn{3}{c}{ De $6 / 1 / 1989$ a $20 / 6 / 2008$} \\
\hline F1 & $3.719(1.359)$ & $0.113(0.071)$ \\
F5 & $3.767(1.260)$ & $0.530(0.114)$ \\
F6 & $3.784(1.171)$ & $0.928(0.144)$ \\
\hline
\end{tabular}

\section{Tabela 3: Estatísticas descritivas dos dados de trigo}

Esta tabela contém as médias e os desvios padrão para o preço e para os anos à maturidade de cada contrato utilizado para fins de estimação.

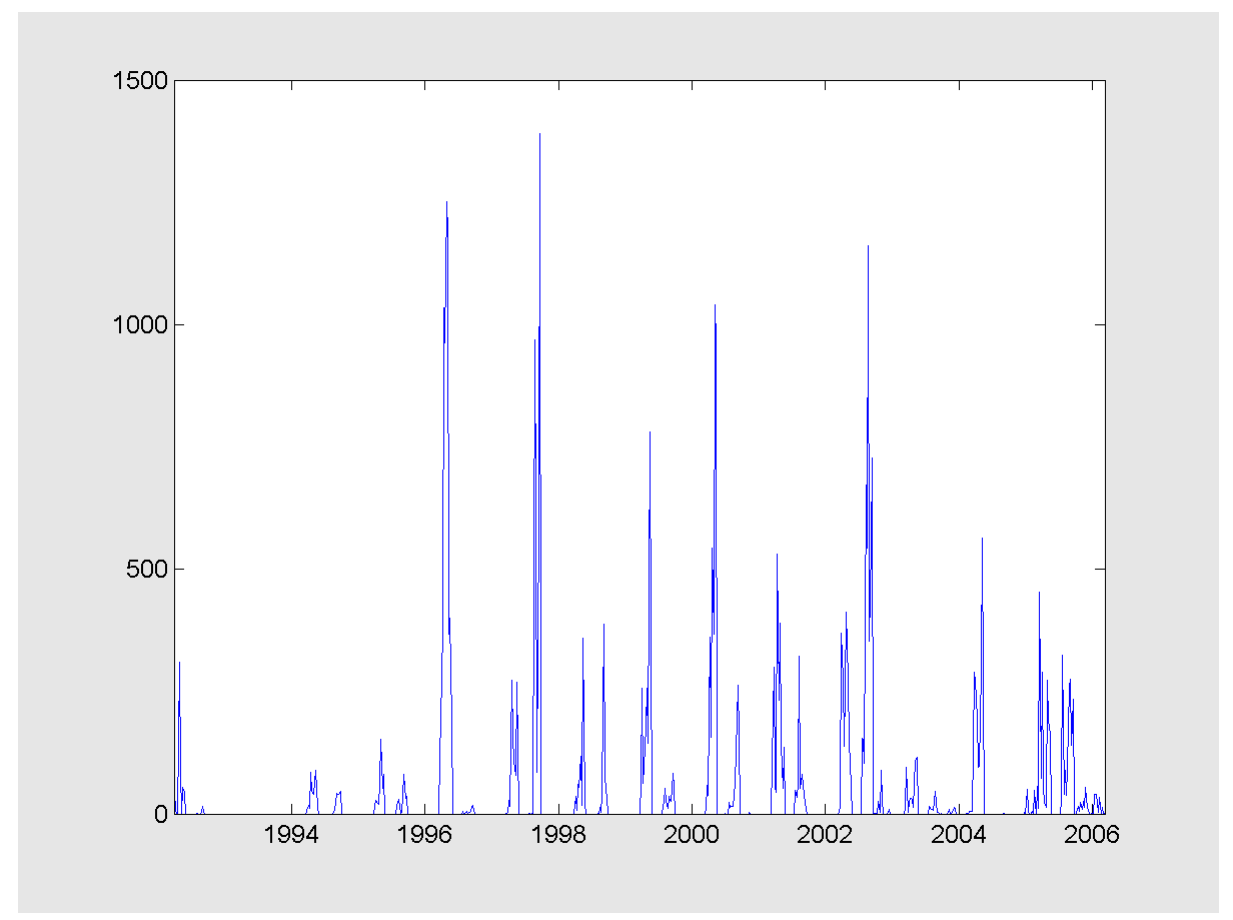

Figura 3: Número de negociações do contrato F7 em um dia

Esta figura mostra mostra o número de negociações diarias do contrato futuro de trigo F7. 
de maneira mais precisa, mas tais contratos não possuem históricos suficientemente longos nos dados colhidos. Schwartz (1997) observa que este problema está presente na maioria das bases de dados públicas provenientes de bolsas de futuros ${ }^{1}$.

As estatísticas básicas da última base de contratos futros utilizada neste trabalho, de cobre, encontram-se na tabela 4. Esta base é, dentre as outras utilizadas neste trabalho, a mais rica em termos de maturidades (o contrato mais longo possui maturidade média de 1.7 anos) e tamanho da amostra (de 1990 a 2012).

\begin{tabular}{ccc} 
Contrato Futuro & $\begin{array}{c}\text { Preço Médio } \\
\text { (Desvio Padrão) }\end{array}$ & $\begin{array}{c}\text { Maturidade Média } \\
\text { (Desvio Padrão) }\end{array}$ \\
\hline \multicolumn{3}{c}{ Data from 5/1/1990 to $24 / 8 / 2012$} \\
F1 & $175.97(117.41)$ & $0.114(0.064)$ \\
F3 & $174.91(117.40)$ & $0.510(0.083)$ \\
F6 & $170.86(115.68)$ & $1.114(0.064)$ \\
F9 & $167.61(112.80)$ & $1.706(0.079)$ \\
\hline
\end{tabular}

\section{Tabela 4: Estatísticas descritivas dos dados de cobre}

Esta tabela contém as médias e os desvios padrão para o preço e para os anos à maturidade de cada contrato utilizado para fins de estimação.

\footnotetext{
${ }^{1} \mathrm{~A}$ análise da literatura feita pelo autor encontrou trabalhos que utilizam amostras com maiores maturidades apenas quando referentes a dados não públicos. Schwartz (1997), por exemplo, utiliza dados proprietários da Enron Capital com maturidades médias de até nove anos.
} 


\section{Resultados}

\section{5..1 Parâmetros Estimados}

Como esperado, os parâmetros para os quais se utilizou um método de Gibbs-Sampling (discriminados no apêndice 7..6) estão associados a cadeias de menor auto-correlação, de aproximadamente zero na vigésima ordem, com exceção dos parâmetros de salto, que apresentam correlação mais intensa. O parâmetro de frequencia de saltos $\phi$, por exemplo, possui uma autocorrelação de aproximadamente 0.6 para a centésima ordem, valor considerado alto pela literatura de métodos de Monte Carlo, o que pode tornar sua convergencia mais lenta e sua estimativa mais imprecisa. Os parâmetros gerados por métodos genéricos de Metropolis Hastings apresentam cadeias com auto-correlação de aproximadamente 0.4 na centésima ordem, valor também considerado alto. Como observa Chib (2001), cadeias de elevada auto-correlação são comuns em simulações de variáveis de altas dimensões, como no presente trabalho. Para maiores informações acerca das cadeias geradas checar o apêndice 7..7, que contém todas as informações relevantes para as simulações de cobre. As demais simulações têm aspecto semelhante.

A tabela 5 contém as médias, os desvios padrão e a significância estatística da distribuição a posteriori de cada pâmetro para os dados de petróleo contidos nos painéis $\mathrm{A}$ e $\mathrm{B}$, onde os parâmetros com subscrito " $m$ " são referentes à reparametrização (explicada no apêndice 7..4) utilizada para a aplicação do algoritmo MCMC.

Os parâmetros de retorno de longo prazo $(\mu)$ são, em todos os casos, significantes e acima da taxa de juros média do período (de 0.03 para o painel A e 0.04 para o painel B). Os valores obtidos neste trabalho oscilam em torno de 0.22 e 0.24 (nota-se que a inclusão de saltos praticamente não altera tais estimativas), valores muito próximos àqueles encontrados por Schwartz (1997), de 0.238 e 0.244 para dois painéis que vão de 1990 a 1995. Estes valores indicam um excesso de retorno da ordem de 0.2.

A estimativa da média de longo prazo do convenience yield, $\alpha$, depende fortemente da 


\begin{tabular}{|c|c|c|c|c|c|c|c|c|}
\hline Base & \multicolumn{4}{|c|}{ Painel A } & \multicolumn{4}{|c|}{ Painel B } \\
\hline Contratos & \multicolumn{4}{|c|}{$\mathrm{F} 1, \mathrm{~F} 5, \mathrm{~F} 10, \mathrm{~F} 15$} & \multicolumn{4}{|c|}{$\mathrm{F} 1, \mathrm{~F} 3, \mathrm{~F} 7, \mathrm{~F} 10$} \\
\hline NOBS & \multicolumn{4}{|c|}{921} & \multicolumn{4}{|c|}{1304} \\
\hline Período & \multicolumn{4}{|c|}{$6 / 1 / 1995$ a $24 / 8 / 2012$} & \multicolumn{4}{|c|}{$4 / 9 / 1987$ a $24 / 8 / 2012$} \\
\hline Modelo & CYES & & $\mathrm{CYE}$ & & CYES & & $\mathrm{CYE}$ & \\
\hline$\mu_{m}$ & $0.274(0.091)$ & $* * *$ & $0.174(0.080)$ & $* *$ & $0.267(0.068)$ & $* * *$ & $0.185(0.078)$ & $* * *$ \\
\hline$\alpha$ & $0.078(0.053)$ & $*$ & $0.078(0.054)$ & $*$ & $0.117(0.045)$ & $* * *$ & $0.119(0.048)$ & $* * *$ \\
\hline$\kappa$ & $1.335(0.054)$ & $* * *$ & $1.337(0.063)$ & $* * *$ & $1.881(0.086)$ & $* * *$ & $1.803(0.109)$ & $* * *$ \\
\hline$\sigma_{S}$ & $0.269(0.015)$ & $* * *$ & $0.318(0.015)$ & $* * *$ & $0.252(0.014)$ & $* * *$ & $0.323(0.016)$ & $* * *$ \\
\hline$\sigma_{\delta}$ & $0.303(0.019)$ & $* * *$ & $0.290(0.018)$ & $* * *$ & $0.392(0.023)$ & $* * *$ & $0.379(0.029)$ & $* * *$ \\
\hline$\rho$ & $0.978(0.008)$ & $* * *$ & $0.856(0.020)$ & $* * *$ & $0.980(0.005)$ & $* * *$ & $0.853(0.016)$ & $* * *$ \\
\hline$\hat{\alpha}$ & $-0.009(0.031)$ & & $-0.008(0.042)$ & & $-0.015(0.055)$ & & $-0.026(0.073)$ & \\
\hline$V$ & $0.020(0.007)$ & $* * *$ & $0.026(0.010)$ & $* * *$ & $0.022(0.010)$ & $* * *$ & $0.027(0.012)$ & $* * *$ \\
\hline$\phi$ & $20.652(3.447)$ & $* * *$ & & & $19.499(2.556)$ & $* * *$ & & \\
\hline $\bar{\eta}_{m}$ & $-0.005(0.004)$ & $*$ & & & $-0.004(0.003)$ & & & \\
\hline$\omega$ & $0.039(0.003)$ & $* * *$ & & & $0.043(0.002)$ & $* * *$ & & \\
\hline$\mu$ & $0.225(0.074)$ & $* * *$ & $0.225(0.080)$ & $* * *$ & $0.233(0.067)$ & $* * *$ & $0.237(0.078)$ & $* * *$ \\
\hline$\lambda$ & $0.115(0.084)$ & $*$ & $0.113(0.089)$ & & $0.249(0.134)$ & $* *$ & $0.260(0.151)$ & $* *$ \\
\hline $\bar{\eta}$ & $-0.004(0.004)$ & & & & $-0.003(0.003)$ & & & \\
\hline
\end{tabular}

\section{Tabela 5: Estimação dos modelos CYE e CYES para dados de petróleo}

Esta tabela contém as médias das distribuições a posteriori para cada parâmetro dos modelos com saltos e sem saltos. Desvios padrão das distribuições a posteriori estão entre parenteses. NOBS = número de observações, $* * *$ indica significância estatística ao nível $99 \%, * *$ indica significância estatística ao nível $95 \%$ e* indica significância estatística ao nível $90 \%$

amostra usada. O painel B, que contempla um intervalo de tempo maior, indica uma valor de aproximadamente 0.12 para $\alpha$, enquanto o painel A, que cobre uma amostra menor, indica um valor bastante menor para este parâmetro, de 0.078. Estas estimativas estão abaixo dos valores encontrados por Schwartz (1997) em painéis que vão de 1990 a 1995, de 0.180 e 1.184, dependendo dos contratos futuros utilizados. Estas diferenças devem-se à grande queda dos preços à vista do petróleo ocorridas em 2008, que foram acompanhados em menor intensidade pelos preços futuros mais longos, gerando uma queda considerável no valor do convenience yield. Isto pode ser visto claramente na figura 4, que contém as estimativas e os intervalos de confiança para estas variáveis latentes: o convenience yield atinge seu mínimo histórico de, aproximadamente, -1 na citada queda de preços.

As estimativas do parâmetro de velocidade de ajustamento $\kappa$ são fortemente significativas e são favoráveis à hipótese de que o convenience yield reverte à uma média de longo prazo. Nossas estimativas são de, aproximadamente, 1.8 para o painel B e 1.4 para o 
painel A. Estes resultados vão ao encontro com aquele obtido por Schwartz (1997) de que estimativas baseadas em contratos de maiores maturidades indicam uma reversão à média menos intensa. Neste sentido, é elucidativa sua estimativa de 1.187 para $\kappa$ utilizando dados de petróleo fornecidos pela Enron Capital, com contatos de maturidades médias de até nove anos.
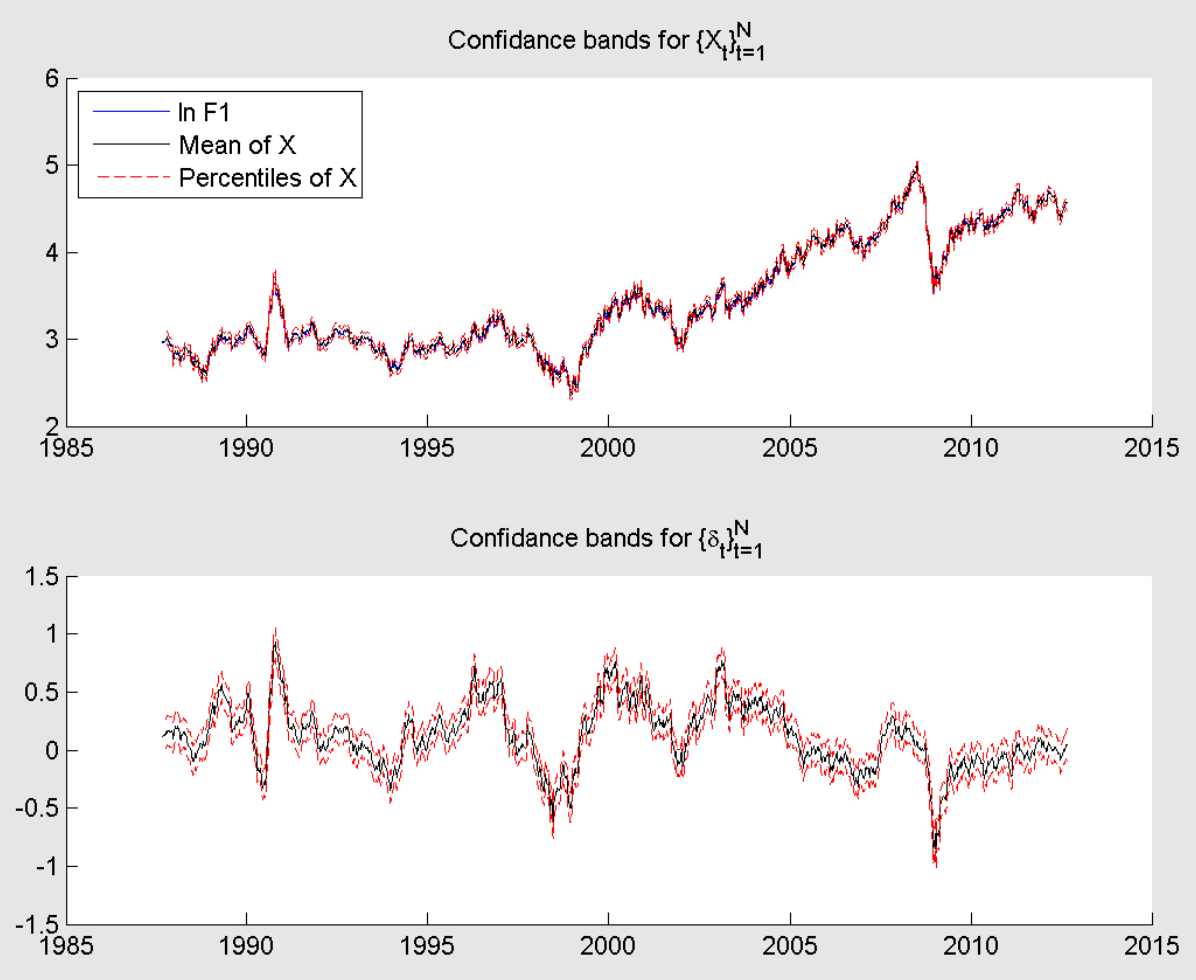

Figura 4: Médias e intervalos de confiança para as variáveis de estado no modelo CYES utilizando os dados de petróleo do painel B

Os resultados obtidos para $\rho$, positivos e estatisticamente significantes, vão ao encontro com a teoria do armazenamento, conforme explicado na seção 2. Nota-se que, em ambos os painéis, a estimativa de $\rho$ aumenta de maneira significante nos modelos com saltos, de 0.86 para 0.98 no painel A e de 0.85 para 0.98 no painel B. A intuição deste resultado é a de que os saltos são capazes de "limpar" os resíduos da equação do preço à vista de choques não correlacionados com o convenience yield. 
O preço do risco do convenience yield, $\lambda_{\delta}$, é positivo, como esperado, e estatisticamente significante, variando entre 0.11 para o painel A e 0.25 para o painel B, valores que diferem muito pouco daqueles encontrados por Schwartz (1997), que variam entre 0.19 e 0.32.

As estimativas obtidas para o parâmetro de frequencia anual de saltos $\phi$, de aproximadamente 20, são altas se comparadas com estudos análogos do mercado de ações, que geralmente o estimam entre 1 e 5; Bjorn Eraker \& Polsonn (2003), por exemplo, encontram valores próximos a 1.5 para o índice S\&P 500. Outro fato interessante a respeito dos parâmetros de saltos é que a estimativa do salto percentual médio, $\bar{\eta}$, é estatisticamente insignificante, indicando a presença de saltos simétricos em dados de petróleo. Este resultado contrasta com a maioria dos estudos de saltos no mercado acionario, que geralmente encontra saltos médios significantemente negativos. Isso é explicado pela assimetria dos log-retornos de petróleo, de aproximadamente -0.26, relativamente baixa se comparada, por exemplo, à assimetria do índice S\&P 500, de -2.6.
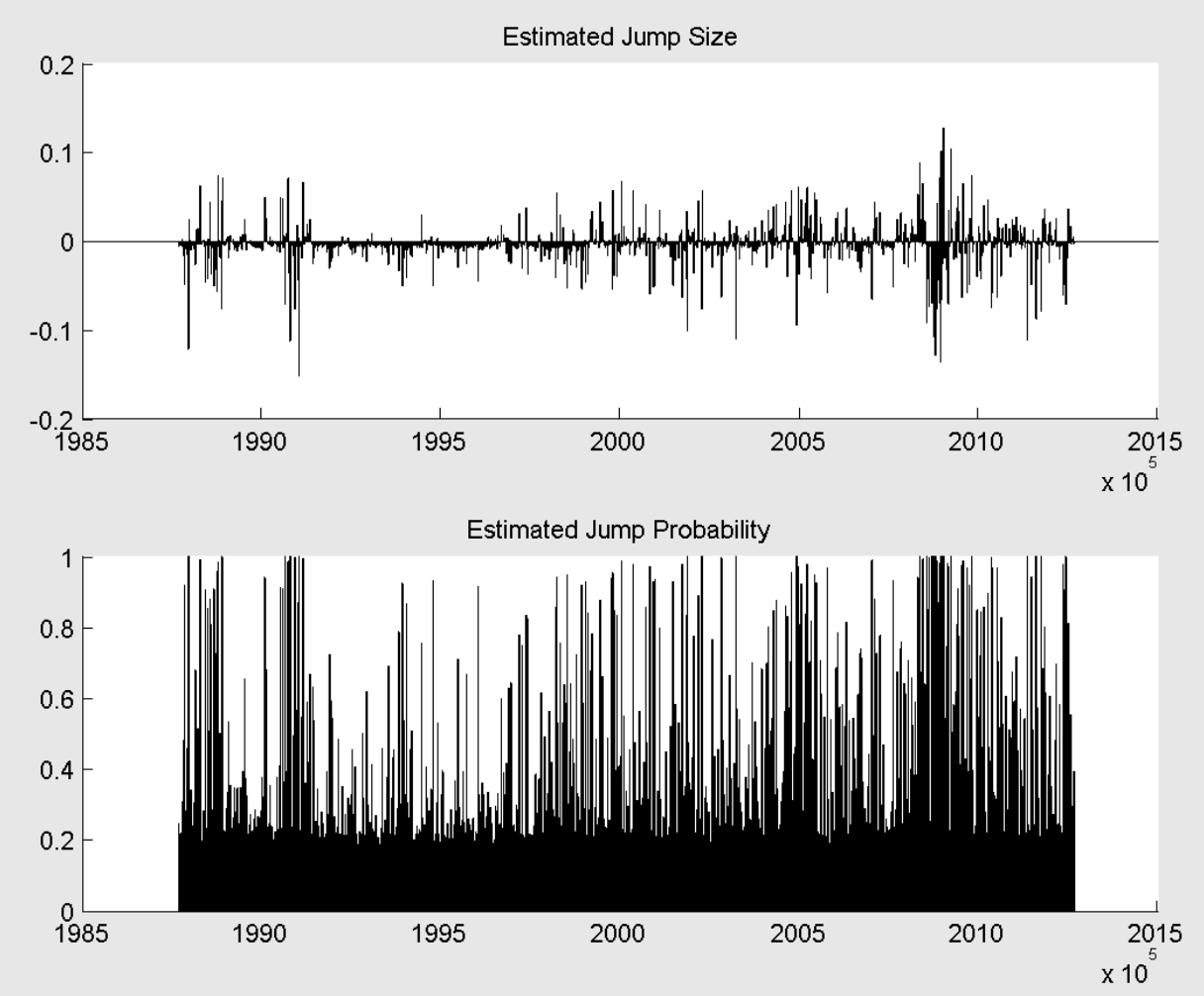

Figura 5: Estimativas da probabilidade e da magnitude dos saltos para dados do painel $\mathrm{B}$ de petróleo 


\begin{tabular}{ccccc} 
Contratos & \multicolumn{4}{c}{$\mathrm{F} 1, \mathrm{~F} 3, \mathrm{~F} 5, \mathrm{~F} 6$} \\
NOBS & $6 / 1 / 1989$ to $20 / 6 / 2008$ \\
Período & CYES & CYE \\
Modelo & $0.012(0.064)$ & & \\
\cline { 2 - 5 }$\mu_{m}$ & $0.127(0.045)$ & $* * *$ & $0.171(0.061)$ & $* * *$ \\
$\alpha$ & $1.538(0.066)$ & $* * *$ & $1.425(0.051)$ & $* * *$ \\
$\kappa$ & $0.216(0.010)$ & $* * *$ & $0.245(0.010)$ & $* * *$ \\
$\sigma_{S}$ & $0.290(0.017)$ & $* * *$ & $0.275(0.021)$ & $* * *$ \\
$\sigma_{\delta}$ & $0.937(0.020)$ & $* * *$ & $0.814(0.024)$ & $* * *$ \\
$\rho$ & $-0.151(0.040)$ & & $-0.166(0.058)$ & \\
$\hat{\alpha}$ & $0.034(0.005)$ & $* * *$ & $0.038(0.010)$ & $* * *$ \\
$V$ & $8.950(2.639)$ & $* * *$ & & \\
$\phi$ & $0.019(0.006)$ & $* * *$ & & \\
$\bar{\eta}_{m}$ & $0.040(0.005)$ & $* * *$ & & \\
$\omega$ & $0.203(0.058)$ & $* * *$ & $0.201(0.061)$ & $* * *$ \\
$\mu$ & $0.427(0.094)$ & $* * *$ & $0.416(0.010)$ & $* * *$ \\
$\lambda$ & $0.020(0.006)$ & $* * *$ & & \\
$\bar{\eta}$ & & & &
\end{tabular}

Tabela 6: Estimação dos modelos CYE e CYES para dados de trigo

Esta tabela contém as médias das distribuições a posteriori para cada parâmetro dos modelos com saltos e sem saltos. Desvios padrão das distribuições a posteriori estão entre parenteses. NOBS = número de observações, *** indica significância estatística ao nível $99 \%$, ** indica significância estatística ao nível $95 \%$ e * indica significância estatística ao nível $90 \%$

A figura 5 mostra as estimativas de probabilidade de salto e de magnitude de salto em cada semana da amostra para o painel B. Nota-se que em alguns períodos, como no final de 2008, há um agrupamento de saltos, indicando um período de alta volatilidade. Como observa Johannes \& Polson (2002), este fenômeno é comum em modelos de saltos que não contemplam volatilidade estocástica e podem indicar um erro de especificação. Como modelos de volatilidade estocástica não estão no escopo deste trabalho, no entanto, não se endereçará aqui este problema.

As tabelas 6 e 7 contém as estimativas referentes aos dados de trigo e cobre, respectivamente. Assim como no caso do petróleo, as estimativas do retorno de longo prazo $\mu$, de 0.12 para cobre e 0.20 para trigo, são estatisticamente significantes e situam-se acima da taxa de juros de livro de risco. A estimatima de $\mu$ aqui encontrada para dados de cobre é sensivelmente distinta àquela de Schwartz (1997), de 0.326; esta divergencia é explicada pela forte queda nos preços e no convenience yield do cobre após 2008, um período não contemplado no trabalho citado; esta queda pode ser vista na figura 6 , que contém as estimativas das variáveis de estado no modelo com saltos. 


\begin{tabular}{ccccc} 
Contratos & \multicolumn{5}{c}{$\mathrm{F} 1, \mathrm{~F} 3, \mathrm{~F} 6, \mathrm{~F} 9$} \\
NOBS & $5 / 1 / 1990$ to $24 / 8 / 2012$ & \\
Período & CYES & CYE \\
Modelo & $0.115(0.056)$ & $* *$ & $0.092(0.079)$ & $*$ \\
\hline$\mu_{m}$ & $0.033(0.045)$ & & $0.033(0.056)$ & \\
$\alpha$ & $0.588(0.060)$ & $* * *$ & $0.646(0.068)$ & $* * *$ \\
$\kappa$ & $0.178(0.020)$ & $* * *$ & $0.237(0.021)$ & $* * *$ \\
$\sigma_{S}$ & $0.092(0.008)$ & $* * *$ & $0.095(0.009)$ & $* * *$ \\
$\sigma_{\delta}$ & $0.829(0.051)$ & $* * *$ & $0.665(0.053)$ & $* * *$ \\
$\rho$ & $0.078(0.158)$ & & $0.078(0.200)$ & \\
$\hat{\alpha}$ & $0.062(0.065)$ & $* * *$ & $0.069(0.084)$ & $* * *$ \\
$V$ & $6.472(3.469)$ & $* * *$ & & \\
$\phi$ & $-0.004(0.009)$ & & & \\
$\bar{\eta}_{m}$ & $0.063(0.011)$ & $* * *$ & & \\
$\omega$ & $0.121(0.058)$ & $* *$ & $0.121(0.079)$ & $*$ \\
$\mu$ & $-0.025(0.090)$ & & $-0.028(0.106)$ & \\
$\lambda$ & $-0.002(0.008)$ & & & \\
$\bar{\eta}$ & & & & \\
\hline
\end{tabular}

\section{Tabela 7: Estimação dos modelos CYE e CYES para dados de cobre}

Esta tabela contém as médias das distribuições a posteriori para cada parâmetro dos modelos com saltos e sem saltos. Desvios padrão das distribuições a posteriori estão entre parenteses. NOBS = número de observações, ${ }^{* * *}$ indica significância estatística ao nível $99 \%$, ** indica significância estatística ao nível $95 \%$ e * indica significância estatística ao nível $90 \%$

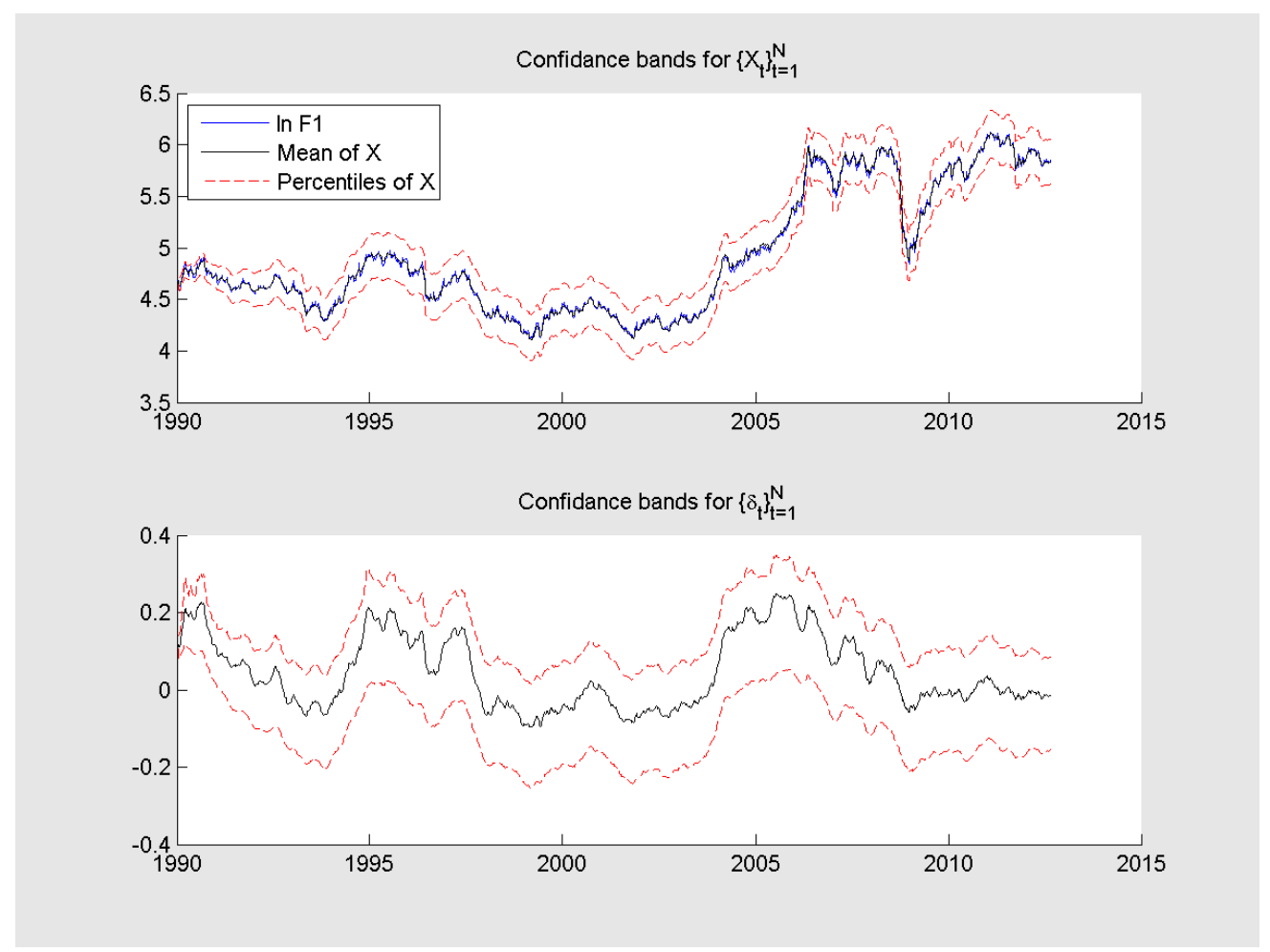

Figura 6: Médias e intervalos de confiança para as variáveis de estado no modelo CYES utilizando os dados de cobre 
Os dados de trigo e cobre também corroboram a hipótese de que o convenience yield reverta à média de longo prazo: os valores de 0.588 e 0.646 para cobre e de 1.538 e 1.425 para trigo são fortemente significantes. As estimativas para o parâmetro de correlação $\rho$, assim como no caso do petróleo, são significantemente positivos e maiores no caso em que saltos são incluídos no modelo, coerentemente com a teoria do armazenamento. A estimativa do preço do risco para os dados de cobre é negativa, mas não é estatisticamente significante; já para os dados de trigo sua estimativa é significante e bastante alta, de 0.427 para o modelo com saltos e 0.416 para o modelo sem saltos.

Os parâmetros de frequencia de saltos estimados para trigo e cobre, de 8.9 e 6.7 respectivamente (ambos estatisticamente significantes), parecem mais coerentes com os estudos de saltos nos mercados de ações e com o conceito de que saltos devem ser eventos raros. Ainda a respeito dos parâmetros de salto, chama a atenção o salto percentual médio $\bar{\eta}$ estimado para os dados de trigo por ser positivo e estatisticamente significante, em contraste com estimativas do mercado acionario, que são usualmente negativas, como já foi exemplificado anteriormente. Uma possível explicação para esta divergencia é a de que os choques de oferta no mercado de trigo são geralmente causados más colheitas, tendo portanto um viés positivo no preço. Não surpreende, portanto, que sua série de retornos tenha uma assimetria positiva, de aproximadamente 0.32. A figura 7 mostra as estimativas da probabilidade de salto e de valor percentual do salto em cada semana da amostra para dados de trigo. Repete-se nesta figura a situação que já foi vista nos saltos para dados de petróleo: há agrupamento de saltos em certos períodos de alta volatilidade, sugerindo que um modelo de volatilidade estocástica poderia se ajustar de maneira mais adequada aos dados. 

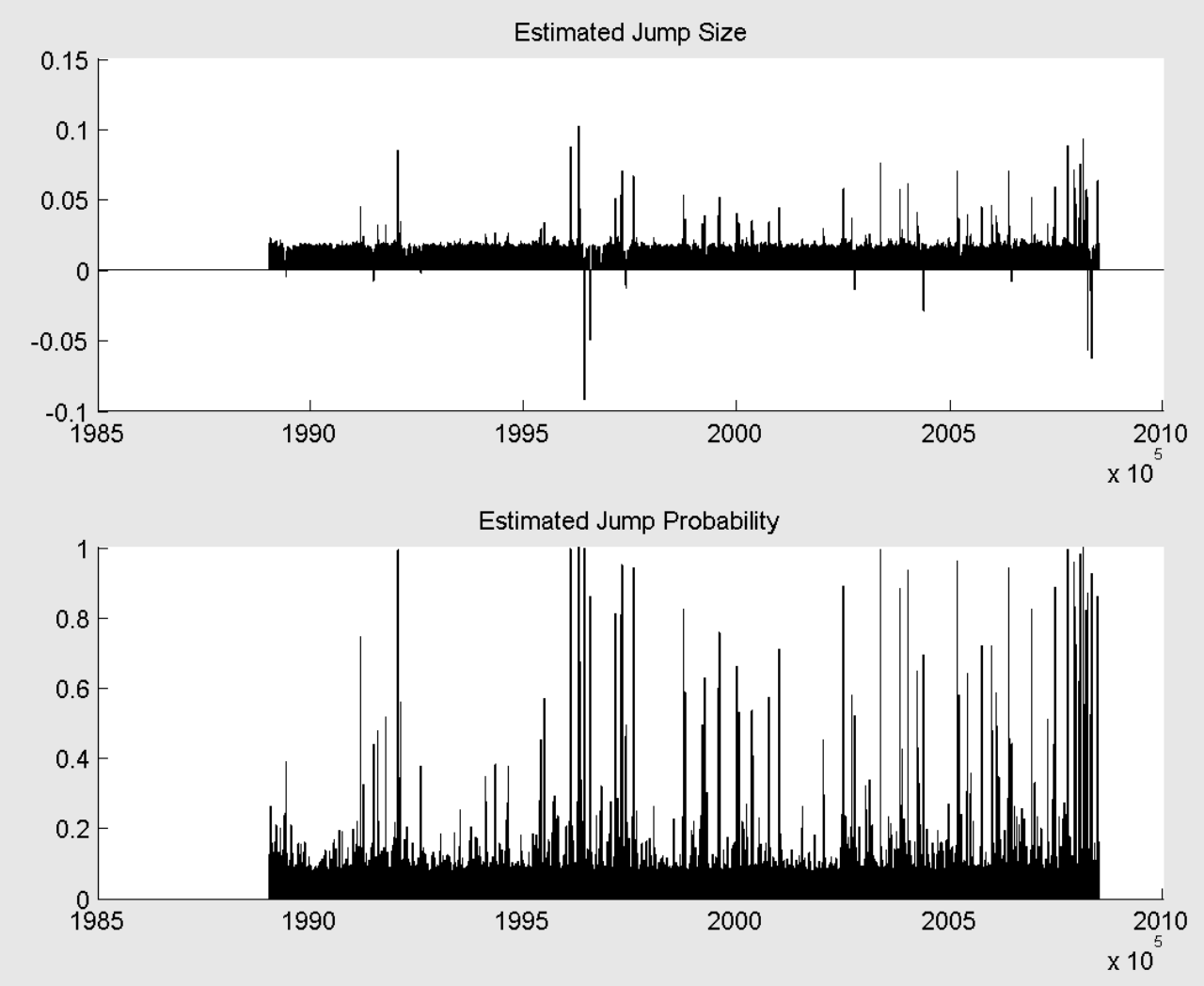

Figura 7: Estimativas da probabilidade e da magnitude dos saltos para dados de trigo 


\section{5..2 Comparação entre os modelos CYE e CYES}

\section{5..2.1 Comparação da REQM para Contratos Futuros Fora da Amostra}

Antes de prosseguir com a comparação dos erros quadráticos médios na previsão dos preços de contratos futuros, faz-se necessário um esclarecimento. Utilizou-se preços de contratos futuros observados no mesmo intervalo de tempo que as observações da amostra de estimação, mas com maturidades distintas. Não se utilizou contratos fora do intervalo de tempo de estimação porque isto necessitaria da estimação adicional da variável de estado nos instantes correspondentes, tornando a comparação muito mais complexa.

A comparação feita aqui não é, portanto, um método de comparação "legitimamente" fora da amostra, pois os contratos futuros de diferentes maturidades apresentam correlação temporal bastante elevada ${ }^{1}$. Não obstante, esta comparação é relevante e será feita nesta subseção.

\begin{tabular}{ccccc}
\hline & \multicolumn{2}{c}{ REQM em Dólares } & \multicolumn{2}{c}{ REQM em Porcentagem } \\
\cline { 2 - 5 } Modelo & CYES & CYE & CYES & CYES \\
\hline F2 & $1.360(0.681)$ & $1.839(0.983)$ & $2.520(1.149)$ & $3.333(1.671)$ \\
F4 & $1.321(0.612)$ & $1.737(0.880)$ & $2.533(0.992)$ & $3.203(1.456)$ \\
F6 & $1.265(0.599)$ & $1.668(0.854)$ & $2.347(0.982)$ & $3.001(1.426)$ \\
F8 & $1.217(0.609)$ & $1.639(0.868)$ & $2.173(1.020)$ & $2.868(1.475)$ \\
F12 & $1.247(0.682)$ & $1.733(0.986)$ & $2.157(1.179)$ & $2.977(1.714)$ \\
F13 & $1.290(0.711)$ & $1.791(1.032)$ & $2.244(1.231)$ & $3.090(1.796)$ \\
F20 & $2.003(1.047)$ & $2.605(1.509)$ & $3.713(1.741)$ & $4.701(2.554)$ \\
F30 & $4.185(2.088)$ & $5.174(2.919)$ & $6.455(2.891)$ & $7.821(4.093)$ \\
Todos & $1.736(1.383)$ & $2.273(1.815)$ & $3.018(2.058)$ & $3.874(2.710)$ \\
\hline
\end{tabular}

Tabela 8: Comparação entre erros de previsão dos modelos CYES e CYE estimados com os dados de petróleo do painel A

Esta tabela apresenta as raízes do erro quadrático médio para um grupo de contratos futuros fora da amostra de estimação no período de 6/1/1995 a 24/8/2012.

A tabela 8 apresenta as raízes dos erros quadráticos médios para um grupo de contratos fora da amostra de estimação, utilizando-se as previsões dos modelos CYE e CYES estimados com os dados do painel A de petróleo. Note que todos os contratos apresentam uma REQM menor para o modelo CYES e, considerando todos os contratos, a REQM (em

\footnotetext{
${ }^{1}$ A correlação entre os preços dos contratos futuros de petróleo F1 e F15, por exemplo, é de 0.988
} 
dólares) é de 2.273 para o modelo CYE e de 1.736 para o modelo CYES, uma diferença de $23 \%$.

Uma das vatagens de se estimar os modelos CYE e CYES por métodos MCMC é a de que os desvios-padrão das raízes quadradas dos erros quadráticos médios são facilmente calculáveis e estão entre parenteses na tabela 8. Observe que, apesar das REQM serem sempre menores no modelo CYES, a diferença nunca supera um desvio padrão. Conclui-se que a pura análise das REQM não agrega evidências a favor do modelo CYES.

As tabelas 9 e 10 contém as RMSE para os dados de trigo e cobre, respectivamente, e contam a mesma história: o modelo CYES logra a obtenção de REQM menores, mas tal descréscimo não é estatisticamente significante.

\begin{tabular}{ccccc}
\hline \multirow{2}{*}{ Modelo } & \multicolumn{2}{c}{ REQM em Dólares } & \multicolumn{2}{c}{ REQM em Porcentagem } \\
\cline { 2 - 5 } & CYES & CYE & CYES & CYES \\
\hline F2 & $0.221(0.048)$ & $0.239(0.073)$ & $4.454(1.114)$ & $5.010(1.789)$ \\
F4 & $0.175(0.041)$ & $0.199(0.068)$ & $4.065(1.067)$ & $4.684(1.763)$ \\
F8 & $0.382(0.145)$ & $0.409(0.197)$ & $8.474(3.285)$ & $9.226(4.558)$ \\
F10 & $0.645(0.234)$ & $0.690(0.326)$ & $14.233(5.053)$ & $15.419(7.138)$ \\
F11 & $0.681(0.264)$ & $0.738(0.372)$ & $16.377(6.251)$ & $17.871(8.892)$ \\
F13 & $1.162(0.394)$ & $1.243(0.561)$ & $24.994(8.336)$ & $27.035(12.010)$ \\
Todos & $0.544(0.405)$ & $0.586(0.479)$ & $12.100(8.874)$ & $13.207(10.606)$ \\
\hline
\end{tabular}

Tabela 9: Comparação entre erros de previsão dos modelos CYES e CYE estimados com os dados de trigo

Esta tabela apresenta as raízes do erro quadrático médio para um grupo de contratos futuros fora da amostra de estimação no período de 6/1/1989 to 20/6/2008

\begin{tabular}{ccccc}
\hline & \multicolumn{2}{c}{ REQM em Dólares } & \multicolumn{2}{c}{ REQM em Porcentagem } \\
\cline { 2 - 5 } Modelo & CYES & CYE & CYES & CYES \\
\hline F2 & $18.028(20.953)$ & $20.175(29.308)$ & $8.708(10.143)$ & $9.776(14.182)$ \\
F4 & $16.620(19.753)$ & $18.761(28.178)$ & $8.098(9.765)$ & $9.173(13.897)$ \\
F5 & $16.517(20.083)$ & $18.758(29.015)$ & $8.116(10.014)$ & $9.249(14.422)$ \\
F10 & $22.020(30.752)$ & $25.770(48.794)$ & $10.962(15.139)$ & $12.842(23.967)$ \\
Todos & $18.296(23.442)$ & $20.866(35.032)$ & $8.971(11.546)$ & $10.260(17.217)$ \\
\hline
\end{tabular}

Tabela 10: Comparação entre erros de previsão dos modelos CYES e CYE estimados com os dados de cobre

Esta tabela apresenta as raízes do erro quadrático médio para um grupo de contratos futuros fora da amostra de estimação no período de 5/1/1990 to 24/8/2012 


\section{5..2.2 Normalidade dos Resíduos}

Considere os resíduos do preço à vista e do convenience yield:

$$
\begin{gathered}
\varepsilon_{X i}=X_{i+1}-X_{i}-\left(\mu-0.5 \sigma_{S}^{2}-\phi \bar{\eta}-\delta\right) \Delta t-\log \left(1+\eta_{i}\right) \Delta q_{i} \\
\varepsilon_{\delta i}=\delta_{i+1}-\delta_{i}-\kappa(\alpha-\delta) \Delta t
\end{gathered}
$$

Estes resíduos não precisam, necessariamente, ser normalmente distribuídos, pois estamos utilizando uma linearização da equação de mudança de estados original. Devem, no entanto, ser aproximadamente normais se o modelo está bem especificado, dado que o erro que se incorre neste tipo de aproximação é pequeno. Um possível método de comparação entre os modelos CYES e CYE é, deste modo, a aderência dos seus resíduos à distribuição normal. Esta subseção é dedicada a esta comparação.
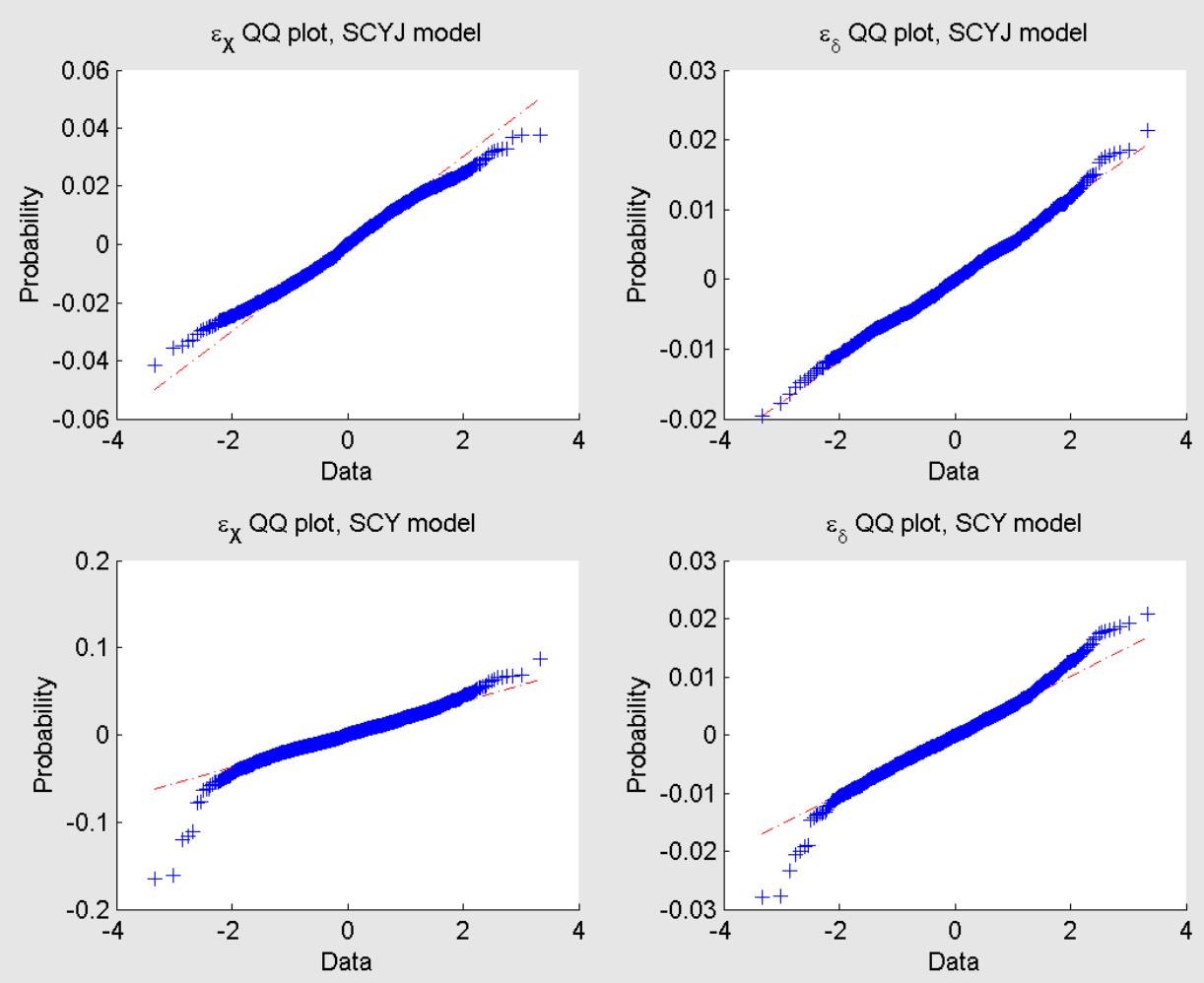

Figura 8: QQ plot para os resíduos das equações de estado para dados de cobre 
Uma primeira comparação neste sentido é a análise dos gráficos dos quantis empíricos dos resíduos de cada modelo contra os previstos numa variavel normal, o QQ plot ${ }^{1}$. A figura 8 exibe o QQ plot dos resíduos de cada uma das variáveis de estado nos modelos CYE e CYES estimados utilizando-se dados de cobre. Nota-se que os saltos conseguem eliminar alguns pontos extremos que estão presentes no QQ plot dos resíduos do logaritmo do preço à vista no modelo CYE. Chama ainda a atenção o fato de que os resíduos da equação do convenience yield ficam mais próximos da normalidade no modelo CYES, como se pode notar nesta mesma figura, mesmo o salto ocorrendo apenas na equação do preço. A figura 9 mostra o QQ plot para os resíduos calculados com dados do painel A de petróleo e tem uma interpretação semelhante.
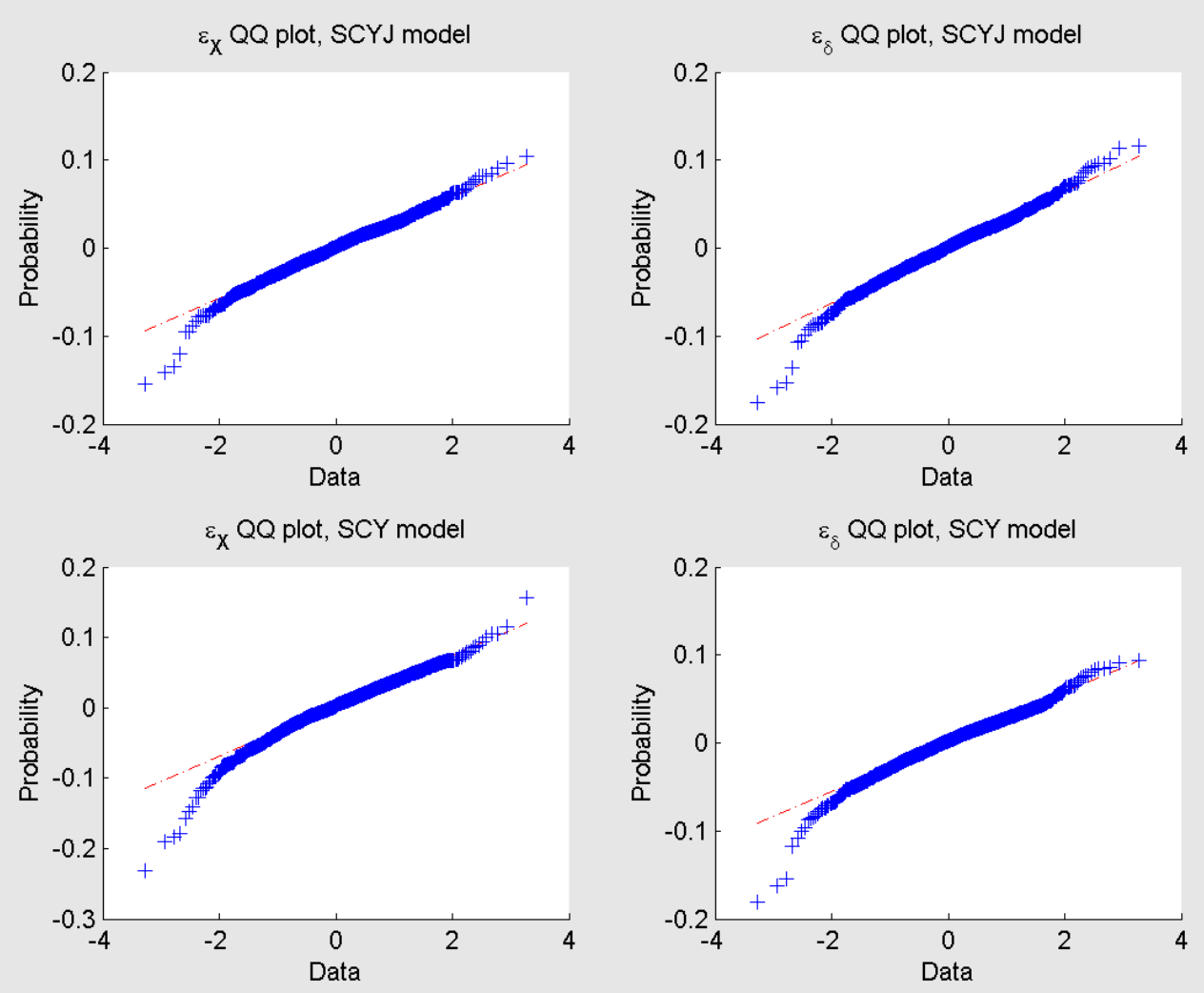

Figura 9: QQ plot para os resíduos das equações de estado para dados de petróleo do painel A

\footnotetext{
${ }^{1}$ Um conjunto de dados será aderente ao modelo normal se seus quantis empíricos forem próximos aos previstos no modelo normal; logo, seus pontos no QQ plot devem estar próximos da diagonal.
} 
Os QQ plots proporcionam uma boa intuição da aderência de uma variável aleatória ao modelo normal, mas não são capazes de responder de maneira objetiva se determinado conjunto de dados pode ser considerado como normalmente distribuído ou não. Para isso precisamos do auxilio de um teste de hipóteses.

Utiliza-se neste trabalho o teste de Jarque-Bera para testar a hipótese nula de normalidade dos resíduos. As tabelas 11 e 12 reportam os valores de assimetria, curtose e p-valor da estatística de Jarque-Bera para os resíduos dos models CYE e CYES.

\begin{tabular}{|c|r|cccc|}
\hline & & \multicolumn{5}{|c|}{ Base de dados } \\
& Modelo & Petróleo (A) & Petróleo (B) & Cobre & Trigo \\
\hline Curtose & CYES & 4.49 & 4.39 & 2.45 & 3.38 \\
\multirow{3}{*}{ Assimetria } & CYE & 5.60 & 6.36 & 9.54 & 3.95 \\
& CYES & -0.39 & -0.15 & 0.02 & 0.29 \\
Estatística JB & CYE & -0.80 & -0.71 & -0.89 & 0.42 \\
p-valor & CYES & 108.19 & 109.91 & 15.20 & 20.69 \\
& CYE & 356.58 & 724.37 & 2260.70 & 67.88 \\
& CYES & $0.00 \%$ & $0.00 \%$ & $0.21 \%$ & $0.00 \%$ \\
& CYE & $0.00 \%$ & $0.00 \%$ & $0.00 \%$ & $0.00 \%$ \\
\hline
\end{tabular}

Tabela 11: Medidas de normalidade para os resíduos da equação do preço à vista

Esta tabela exibe as medidas de normalidade dos resíduos para cada modelo (CYES e CYE) e cada base de dados. As colunas correspondem às bases de dados utilizadas, sendo petróleo (A) o painel A de petróelo e petróleo (B) o painel $\mathrm{B}$ de petróelo. O p-valor exibido nas últimas linhas é referente à hipótese nula de normalidade para o teste de Jarque-Bera.

\begin{tabular}{|c|r|cccc|}
\hline & & \multicolumn{5}{|c|}{ Base de dados } \\
& Modelo & Petróleo (A) & Petróleo (B) & Cobre & Trigo \\
\hline Curtose & CYES & 4.70 & 4.58 & 3.31 & 3.82 \\
& CYE & 5.68 & 6.28 & 4.72 & 3.89 \\
Assimetria & CYES & -0.38 & -0.15 & 0.19 & 0.46 \\
& CYE & -0.68 & -0.47 & 0.00 & 0.50 \\
Estatística JB & CYES & 133.25 & 140.20 & 12.22 & 63.60 \\
& CYE & 346.39 & 631.78 & 145.97 & 76.25 \\
& CYES & $0.00 \%$ & $0.00 \%$ & $0.49 \%$ & $0.00 \%$ \\
& CYE & $0.00 \%$ & $0.00 \%$ & $0.00 \%$ & $0.00 \%$ \\
\hline
\end{tabular}

Tabela 12: Medidas de normalidade para os resíduos da equação do convenience yield 
valores destas variáveis em uma distribuição normal (0 e 3, respectivamente), mas não o suficiente para que os testes de Jarque-Bera não rejeitem a hipótese nula de normalidade, como pode-se ver nas linhas que contém os p-valores, sempre muito próximos de zero. Estes resultados não dão indícios de que o modelo CYES está melhor especificado em termos da normalidade dos resíduos.

\section{5..2.3 Comparação das REQM para Preços de Opções}

Nesta subseção descreve-se a comparação dos modelos CYES e CYE em termos dos seus erros de previsão para preços de opções americanas fora da amostra de estimação.

Para este fim se utilizou uma base de dados de preços de opções americanas de compra de contratos futuros de petróleo consistindo de um total de 1680 observações mensais de 67 contratos distintos no intervalo de tempo de 1/5/2009 a 27/4/2012. Esta amostra contempla opções com diferentes maturidades e diferentes preços de exercício.

Para fins de ilustração se exibe na figura 10 a evolução histórica dos preços de todas as 67 opções de contratos futuros de petróleo utilizadas nesta subseção, assim como o preço do contrato futuro mais curto, F1. Como esperado, os preços de opções apresentam certa correlação com F1. Esta correlação, no entanto, da ordem de 0.7, não é tão alta quanto a que occorre entre contratos futuros (da ordem de 0.98), e permite que opções tenham uma dinâmica própria. A comparação entre os modelos CYES e CYE com base em preços de opções pode, portanto, ser considerada uma comparação legitimamente fora da amostra.

A tabela 13 mostra as raízes quadradas dos erros quadráticos médios das previsões dos preços das opções americanas em termos monetários (em dólares) e em termos percentuais para ambos os modelos CYES e CYE. Os preços previstos pelos modelos foram calculados por meio do método LSM.

Nota-se, primeiramente, que as REQM da tabela 13 são excessivamente altas, ultrapassando $700 \%$ para ambos os modelos com saltos e sem saltos, numa clara indicação de que nenhum destes modelos é capaz de explicar os preços de opções observados no mercado de 

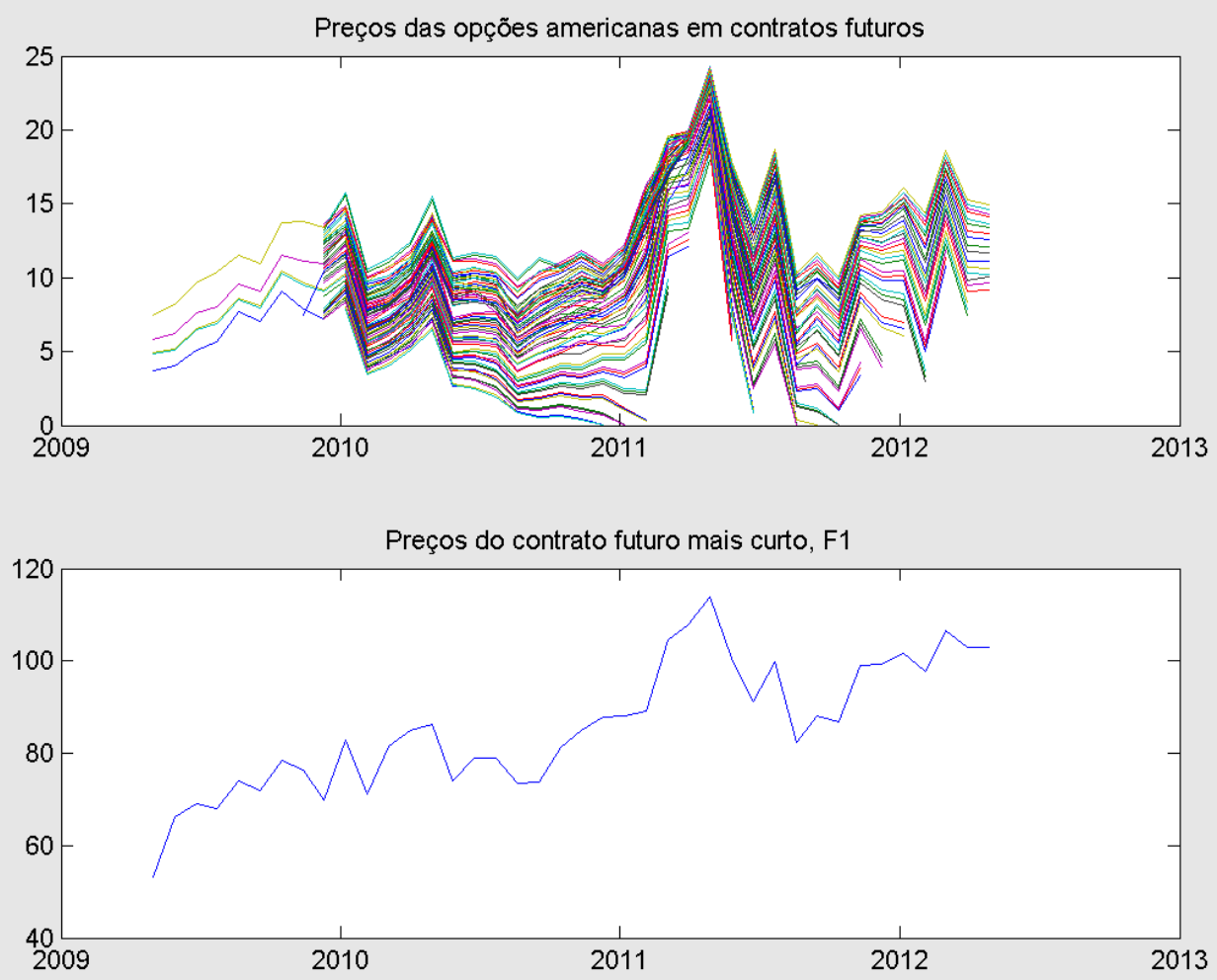

Figura 10: Evoulção histórica da amostra de opções americanas de petróleo e do contrato futuro F1

\begin{tabular}{ccc}
\hline & Dólares & Porcentagem \\
\hline CYES & 8.046 & 759.861 \\
CYE & 9.067 & 710.300 \\
\hline
\end{tabular}

Tabela 13: Comparação entre erros de previsão dos modelos CYES e CYE para dados de opções de futuros de petróleo

Esta tabela apresenta a comparação das raízes quadradas dos erros quadráticos médios para ambos os modelos CYE e CYES utilizando 67 contratos no intervalo 1/5/2009 to 27/4/2012. O tamanho da amostra é 1680 . 
maneira apropriada. Observe-se ainda que os resultados são, a primeira vista, ambíguos: a REQM é menor para o modelo CYES em termos monetarios e maior para o mesmo modelo em termos percentuais.

A explicação deste resultado aparentemente contraditorio pode ser encontrada na figura 11, que contém os preços de mercado e os preços estimados para a opção CLF1C_95 $5^{1}$. Note que os preços previstos segundo o modelo CYES são, em geral, maiores que aqueles do modelo CYE, sendo ambos os modelos incapazes de acompanhar a evolução temporal dos dados de mercado; estes são, na maior parte do tempo, superiores a ambas as previsões, fazendo com que a REQM seja menor no modelo CYES. Em certos períodos de tempo, no entanto, os preços de mercado aproximam-se de zero de maneira muito mais intensa que as previsões de ambos os modelos, fazendo que com o erro percentual do modelo CYES seja muito mais elevado que aquele do modelo CYE, explicando porque este modelo logra a obtenção de uma REQM menor em termos percentuais.

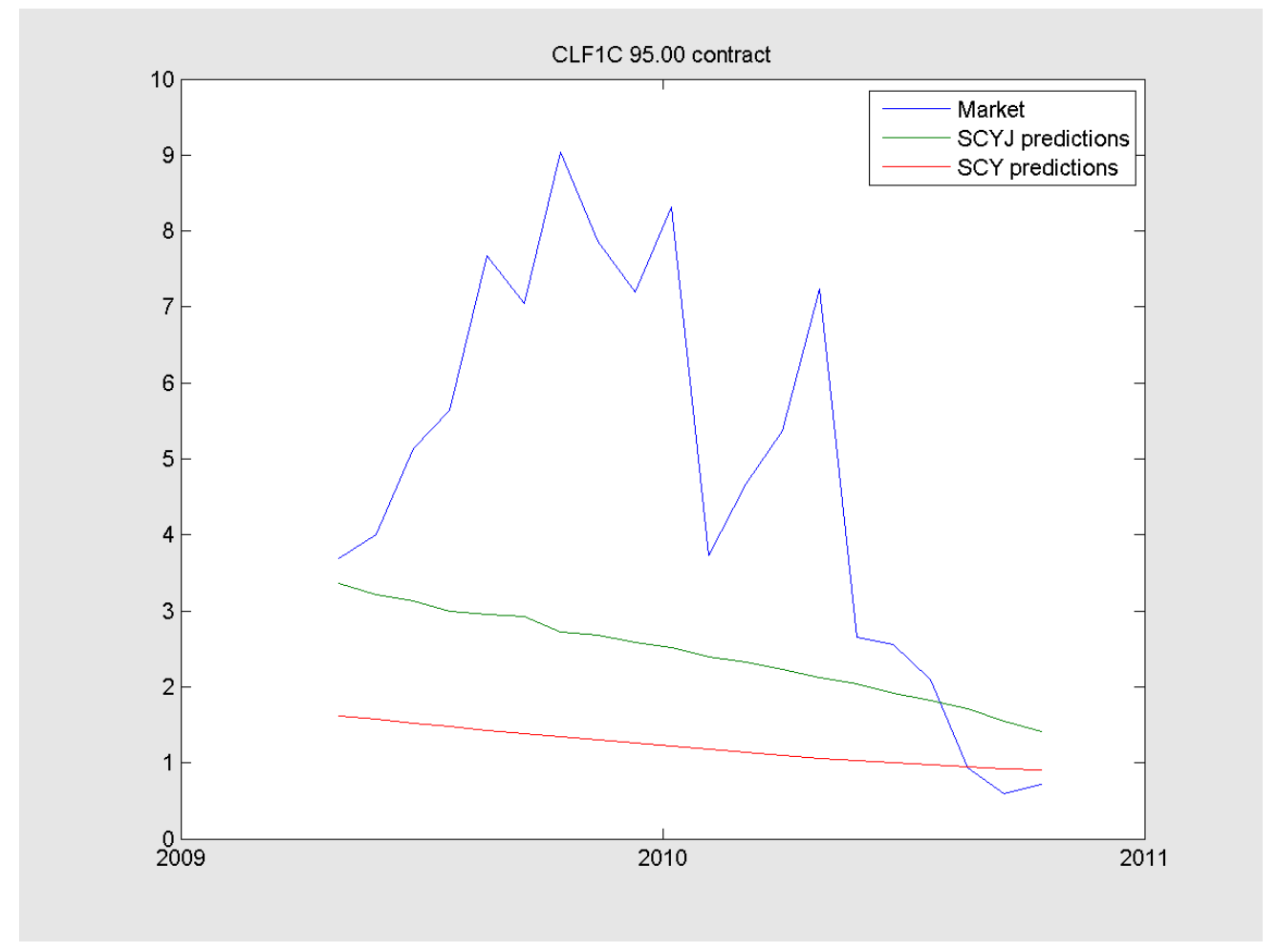

Figura 11: Dados de mercado e previsões para o contrato CLF1C 95.00

\footnotetext{
${ }^{1}$ CLF1C_95 é o código Bloomberg do contrato em questão. "CL" é o código para futuros de petróleo, "F1" é o código para o mês de maturidade de Janeiro de 2011, "C" é o código para uma opção de compra e $95 \%$ é a razão strike / preço de exercício.
} 
Conclui-se que o modelo CYES não apresenta maior aderência aos dados de opções de contratos futuros de petróleo. Tanto o modelo sem saltos quanto o modelo com saltos mostram-se incapazes de explicar a variação temporal dos preços de opções, sugerindo que fatores de risco não contemplados no presente trabalho podem explicar sua dinâmica. 


\section{Conclusão}

Com motivação nas contribuições recentes da literatura de derivativos de ações, como Gurdip Bakashi \& Chen (1997), Bates (1991) e Pan (2002), que encontra um prêmio de risco de saltos relevante na explicação de preços de opções, este trabalho verifica a relvancia de saltos no apreçamento de derivativos de commodities. Para tanto, estimou-se um modelo de convenience yield estocástico e verificou-se o impacto da inclusão de saltos no preço à vista através de diversas medidas.

Em nenhum dos criterios utilizados neste trabalho encontrou-se evidências de que saltos podem apreçar derivativos com maior precisão ou gerar ganhos em termos da correta especificação do modelo: (1) a comparação de erros de previsão para contratos futuros mostrou uma pequena, porém estatisticamente insignificante, vantagem do modelo com saltos; (2) a comparação dos erros de previsão para dados de opções não mostrou melhor adarencia de nenhum dos modelos; e (3) a análise de normalidade dos resíduos mostrou que a curtose e a assimetria são reduzidas com a inclusão de saltos, mas a hipótese nula de normalidade é rejeitada em todos os modelos.

Estes resultados não rejeitam a hipótese de trajetórias continuas para preços à vista feita pela grande maioria da literatura de apreçamento de commodities, como Black (1976), Gibson \& Schwartz (1990) e Schwartz (1997), sedimentando caminho seguro para trabalhos futuros que venham a fazer este pressuposto.

Este trabalho também mostra que um modelo de dois fatores com convenience yield estocástico não é capaz de descrever a dinâmica de preços de opções de maneira adequada, sugerindo a presença de fatores de risco não contemplados e gerando insumos para pesquisas futuras. A análise da formação de grupos de saltos concentrados em determinados períodos de tempo dão indícios de que um modelo de volatilidade estocástica poderia se ajustar de maneira mais adequada aos dados e, eventualmente, apreçar derivativos de commodities com maior precisão. 


\section{Apêndice}

\section{7..1 Apreçamento de contratos futuros}

Trata-se aqui do apreçamento do contratos futuros no modelo CYE. Supõe-se que as variáveis de estado $S, \delta$ seguem o processo

$$
\begin{gathered}
d S=\mu_{S} S d t+\sigma_{S} S d Z_{S} \\
d \delta=\mu_{\delta} d t+\sigma_{\delta} d Z_{\delta}
\end{gathered}
$$

onde $Z_{S}$ e $Z_{\delta}$ são movimentos Brownianos correlacionados por

$$
d Z_{S} d Z_{\delta}=\rho_{S \delta} d t
$$

Sejam $F^{1}$ e $F^{2}$ os preços de dois contratos futuros da commodity. Denote por $H$ o valor de um portfolio auto-financiável com $\omega_{1}$ unidades de $F^{1}, \omega_{2}$ unidades de $F^{2}$ e $\omega_{S}$ unidades da commodity. Defina

$$
\omega_{1}=F_{\delta}^{2}, \omega_{2}=-F_{\delta}^{1}, \omega_{S}=F_{S}^{2} F_{\delta}^{1}-F_{S}^{1} F_{\delta}^{2}
$$

Pelo Lemma de Ito verifica-se

$$
\begin{aligned}
d H= & {\left[\omega_{1} \mu_{F^{1}} F^{1}+\omega_{2} \mu_{F^{2}} F^{2}+\omega_{S}\left(\mu_{S}+\delta\right) S\right] d t+} \\
& {\left[\omega_{1} F_{S}^{1}+\omega_{2} F_{S}^{2}+\omega_{S}\right] S \sigma_{S} d Z_{S}+\left[\omega_{1} F_{\delta}^{1}+\omega_{2} F_{\delta}^{2}\right] \sigma_{c} d Z_{c} } \\
= & {\left[\omega_{1} \mu_{F^{1}} F^{1}+\omega_{2} \mu_{F^{2}} F^{2}+\omega_{S}\left(\mu_{S}+\delta\right) S\right] d t }
\end{aligned}
$$

onde

$$
\mu_{F^{j}}=\frac{F_{S}^{j} \mu_{S} S+F_{\delta}^{j} \mu_{\delta}+\frac{1}{2} F_{S S}^{j} \sigma_{S}^{2} S^{2}+\frac{1}{2} F_{\delta \delta}^{j} \sigma_{\delta}^{2}+F_{t}^{j}+F_{S \delta}^{j} S \sigma_{S} \sigma_{\delta} \rho_{S \delta}}{F^{j}}
$$


para $j \in\{1,2\}$, o que mostra que o portfolio em questão é livre de risco. Logo, por não arbitragem verifica-se $d H=r H d t$, isto é,

$$
F_{\delta}^{2} \mu_{F^{1}} F^{1}-F_{\delta}^{1} \mu_{F^{2}} F^{2}+\left(F_{S}^{2} F_{\delta}^{1}-F_{S}^{1} F_{\delta}^{2}\right)\left(\mu_{S}+\delta-r\right) S=0
$$

de modo que

$$
F_{\delta}^{2}\left[F^{1} \mu_{V^{1}}-F_{S}^{1} S\left(\mu_{S}+\delta-r\right)\right]=F_{\delta}^{1}\left[F^{2} \mu_{F^{2}}-F_{S}^{2} S\left(\mu_{S}+\delta-r\right)\right]
$$

e que

$$
\begin{aligned}
& \frac{F^{1} \mu_{V^{1}}-F_{S}^{1} S\left(\mu_{S}+\delta-r\right)}{F_{\delta}^{1}}= \\
& \frac{F^{2} \mu_{V^{2}}-F_{S}^{2} S\left(\mu_{S}+\delta-r\right)}{F_{\delta}^{2}} \doteq \lambda_{\delta} .
\end{aligned}
$$

Isto é, o preço do risco do convenience yield, $\lambda_{\delta}$, é independente do específico contrato futuro utilizado, dependendo apenas do tempo e das variáveis de estado.

Suopõe-se nas próximas linhas que $\lambda_{\delta}$ é constante. Assim, para um dado contrato $F=$ $F(t, S, \delta)$ deve valer que

$$
F \mu_{F}-F_{S} S\left(\mu_{S}+\delta-r\right)-F_{\delta} \lambda_{\delta}=0
$$

Substituíndo o valor $\mu_{V}$ por 7.34 obtém-se a seguinte equação de derivadas parciais:

$$
\frac{1}{2} F_{S S} \sigma_{S}^{2} S^{2}+\frac{1}{2} F_{\delta \delta} \sigma_{\delta}^{2}+F_{S \delta}^{j} S \sigma_{S} \sigma_{\delta} \rho_{S \delta}+F_{S} S(r-\delta)+F_{\delta}\left(\mu_{c}-\lambda_{\delta}\right)+F_{t}=0
$$

O movimento Browniano na medida neutra ao risco toma a forma 


$$
d Z_{S}^{*}=d Z_{S}+\frac{\mu_{S}+\delta-r}{\sigma_{S}} d t \text { and } d Z_{\delta}^{*}=d Z_{\delta}+\lambda_{\delta} d t
$$

de modo que a lei de movimento na medida neutra ao risco é

$$
\begin{aligned}
& d S=(r-\delta) d t+\sigma_{S} S d Z_{S}^{*} \\
& d \delta=\left(\mu_{\delta}-\lambda_{\delta}\right) d t+\sigma_{\delta} d Z_{\delta}^{*}
\end{aligned}
$$

A hipótese de que $\delta$ segue um processo de reversão à média é

$$
\mu_{\delta}=\kappa(\alpha-\delta)
$$

e o modelo fica

$$
\begin{array}{r}
d S=(r-\delta) d t+\sigma_{S} S d Z_{S}^{*} \\
d \delta=\kappa(\hat{\alpha}-\delta) d t+\sigma_{c} d Z_{c}^{*}
\end{array}
$$

onde $\hat{\alpha}=\alpha-\frac{\lambda_{\delta}}{\kappa}$.

Seja $F(T, S, \delta)$ o preço de um contrato futuro com maturidade $T$. Pelo Lemma de Ito,

$$
d[\ln (S)]=\left(r-\delta-0.5 \sigma_{S}^{2}\right) d t+\sigma_{S} d Z_{S}^{*}
$$

de modo que

$$
\ln (S(T))=\ln (S(0))+\int_{0}^{T} r(\nu) d \nu-\int_{0}^{T} \delta(\nu) d \nu-0.5 \sigma_{S}^{2} T+\sigma_{S} \int_{0}^{T} d Z_{S}^{*}(\nu)
$$

Defina

$$
X(T)=\ln (S(T))-\int_{0}^{T} r(\nu) d \nu
$$


donde

$$
X(T)=\ln (S(0))-\int_{0}^{T} \delta(\nu) d \nu-0.5 \sigma_{S}^{2} T+\sigma_{S} \int_{0}^{T} d Z_{S}^{*}(\nu)
$$

Logo, como $X(T)$ é normalmente distribuído, deve-se ter

$$
F(t, T)=\hat{\mathbf{E}}_{0}\left[e^{X(T)+\int_{0}^{T} r(\nu) d \nu}\right]=e^{\hat{\mathbf{E}}_{0}[X(T)]+\frac{1}{2} \operatorname{Var}_{0}[X(T)]+r T}
$$

O cálculo de $\hat{\mathbf{E}}_{t}[X(T)]$ pode ser efetuado da seguinte maneira. Primeiramente, verifica-se

$$
\hat{\mathbf{E}}_{0}[X(T)]=\ln (S(0))-0.5 \sigma_{S}^{2} T-\int_{0}^{T} \hat{\mathbf{E}}_{0}[\delta(\nu)] d \nu .
$$

Para calcular a equação acima utilizamos a soulução do processo de reversão à média que rege o movimento de $\delta$

$$
d \delta=\kappa(\hat{\alpha}-\delta) d t+\sigma_{c} d Z_{c}^{*}
$$

de modo que

$$
\delta(t)=\delta(0) e^{-\kappa t}+\hat{\alpha}\left(1-e^{-\kappa t}\right)+\sigma_{\delta} \int_{0}^{t} e^{\kappa(\nu-t)} d Z_{c}^{*}(\nu)
$$

o que implica que

$$
\begin{gathered}
\hat{\mathbf{E}}_{0}[\delta(\nu)]=\hat{\alpha}+(\delta(0)-\hat{\alpha}) e^{-\kappa \nu} \\
\int_{0}^{T} \hat{\mathbf{E}}_{0}[\delta(\nu)] d \nu=\hat{\alpha} T+\frac{(\delta(0)-\hat{\alpha})}{\kappa}\left(1-e^{-\kappa T}\right),
\end{gathered}
$$

donde 


$$
\hat{\mathbf{E}}_{0}[X(T)]=\ln (S(0))-\left(\alpha+0.5 \sigma_{S}^{2}\right) T-\frac{(\delta(0)-\hat{\alpha})}{\kappa}\left(1-e^{-\kappa T}\right) .
$$

Calcula-se agora $\operatorname{var}_{0}[X(T)]$. Primeiramente,

$$
\begin{aligned}
\operatorname{var}_{0}[X(T)] & =\operatorname{var}_{t}\left[\int_{0}^{T} \delta(\nu) d \nu\right]+\sigma_{S}^{2} \operatorname{var}_{0}\left[\int_{0}^{T} d Z_{S}^{*}(\nu)\right] \\
& -2 \sigma_{S} \operatorname{cov}_{0}\left[\int_{0}^{T} \delta(\nu) d \nu, \int_{0}^{T} d Z_{S}^{*}(\nu)\right] .
\end{aligned}
$$

É fácil ver que

$$
\operatorname{var}_{0}\left[\int_{0}^{T} d Z_{S}^{*}(\nu)\right]=T
$$

de modo que

$$
\begin{aligned}
\operatorname{var}_{0}\left[\int_{0}^{T} \delta(\nu) d \nu\right] & =\operatorname{var}_{0}\left[\sigma_{\delta} \int_{0}^{T} \int_{0}^{\nu} e^{\kappa(s-\nu)} d Z_{\delta}^{*}(s) d \nu\right] \\
& =\operatorname{var}_{0}\left[\sigma_{\delta} \int_{t}^{T} \int_{s}^{T} e^{\kappa(s-\nu)} d \nu d Z_{\delta}^{*}(s)\right] \\
& =\operatorname{var}_{0}\left[\sigma_{\delta} \int_{0}^{T} \frac{1}{\kappa}\left(1-e^{\kappa(s-T)}\right) d Z_{c}^{*}(s)\right] \\
& =\sigma_{c}^{2} \hat{\mathbf{E}}_{0}\left[\int_{0}^{T} \frac{1}{\kappa}\left(1-2 e^{\kappa(s-T)}+e^{2 \kappa(s-T)}\right) d Z_{c}^{*}(s)^{2}\right] \\
& =\frac{\sigma_{c}^{2}}{\kappa^{2}} T-2 \frac{\sigma_{\delta}^{2}}{\kappa^{3}}\left(1-e^{-\kappa T}\right)+\frac{1}{2} \frac{\sigma_{\delta}^{2}}{\kappa^{3}}\left(1-e^{-2 \kappa T}\right)
\end{aligned}
$$




$$
\begin{aligned}
\operatorname{cov}_{0}\left[\int_{0}^{T} \delta(\nu) d \nu, \int_{0}^{T} d Z_{S}^{*}(\nu)\right] & =\sigma_{\delta} \hat{\mathbf{E}}_{0}\left[\sigma_{c} \int_{0}^{T} \frac{1}{\kappa}\left(1-e^{\kappa(s-T)}\right) d Z_{c}^{*}(s) \ldots\right. \\
& \left.\ldots \int_{0}^{T} d Z_{S}^{*}(\nu)\right] \\
& =\sigma_{\delta} \hat{\mathbf{E}}_{0}\left[\sigma_{\delta} \int_{t}^{T} \frac{1}{\kappa}\left(1-e^{\kappa(s-T)}\right) d Z_{c}^{*}(s) d Z_{S}^{*}(\nu)\right] \\
& =\frac{\sigma_{\delta} \rho_{S \delta}}{\kappa}\left(T-\frac{1}{\kappa}\left(1-e^{-\kappa T}\right)\right)
\end{aligned}
$$

e então

$$
\begin{aligned}
\operatorname{var}_{0}[X(T)] & =\frac{\sigma_{\delta}^{2}}{\kappa^{2}} T-2 \frac{\sigma_{\delta}^{2}}{\kappa^{3}}\left(1-e^{-\kappa T}+\frac{1}{2} \frac{\sigma_{\delta}^{2}}{\kappa^{3}}\left(1-e^{-2 \kappa T}\right)\right. \\
& +\sigma_{S}^{2} T-2 \frac{\sigma_{\delta} \sigma_{S} \rho_{S \delta}}{\kappa}\left(T-\frac{1}{\kappa}\left(1-e^{-\kappa T}\right)\right)
\end{aligned}
$$

Finalmente,

$$
\begin{aligned}
\log (F(T)) & =\hat{\mathbf{E}}_{0}[X(T)]+\frac{1}{2} \operatorname{var}_{0}[X(T)] \\
& =\log (S)-\delta \frac{1-e^{-\kappa T}}{\kappa}+A(T)
\end{aligned}
$$

onde

$$
\begin{aligned}
A(T) & =\left(r-\hat{\alpha}+\frac{1}{2} \frac{\sigma_{c}^{2}}{\kappa}-\frac{\sigma_{c} \sigma_{S} \rho_{S c}}{\kappa}\right) T+\frac{1}{4} \sigma_{\delta}^{2} \frac{1-e^{-2 \kappa T}}{\kappa^{3}} \\
& +\left(\hat{\alpha} \kappa+\sigma_{\delta} \sigma_{S} \rho_{S c}-\frac{\sigma_{\delta}}{\kappa}\right) \frac{1-e^{-\kappa T}}{\kappa^{2}}
\end{aligned}
$$




\section{7..2 Algoritmo LSM}

Esta seção é destinada à uma precisa descrição do algoritmo de mínimos quadrados de Monte Carlo (LSM na sigla em inglês) utilizado para o cálculo dos preços de opções americanas neste trabalho. Assume-se um espaço de probabilidade completo $(\Omega, \mathbf{F}, P)$ em um horizonte de tempo finito $\left[0, T_{1}\right]$, onde $\Omega$ é o conjunto de todas as possíveis realizações $\omega$ de trajetórias das variáveis de estado em $\left[0, T_{1}\right], \mathbf{F} \subset 2^{\Omega}$ é a $\sigma$-álgebra de conjuntos menuraveis de $\Omega$ e $P: \mathbf{F} \rightarrow[0,1]$ é a medida de probabilidade.

Suponha que se deseja calcular o valor de um ativo contingente que pode ser exercidos em todos os pontos do conjunto $S \subset\left[0, T_{1}\right]$ com a obtenção de um payoff $f(t, \omega)$. No caso de uma opção de um contrato futuro com maturidade $T$ e preço strike $K$ tem-se

$$
\begin{gathered}
S=\left[0, T_{1}\right] \\
f\left(t, X_{t}, \delta_{t}\right)=\max \left[F\left(t, T, X_{t}, \delta_{t}\right)-K, 0\right] .
\end{gathered}
$$

A primeira etapa da aplicação do algoritmo LSM é a aproximação do ativo contingente por um outro ativo, conhecido como opção de bermuda, exercível apenas num subconjunto finito $\left\{t_{1}, t_{2}, \ldots, t_{K}\right\} \subset S$. Aqui deve-se utilizar um número suficientemente grande de pontos para tornar o erro de aproximação desprezível (para mais detalhes, Longstaff \& Schwartz (2001)).

Para cada $t_{i}$, seja $M_{t_{i}}(\omega)$ o valor de continuação (isto é, o valor de não exercício) da opção de bermuda em $t_{i}$. Suporemos que cada função $M_{t_{i}}$ pode ser escrita como uma combinação de funções básicas $\left\{L_{j}\right\}_{j=0}^{\infty}$ :

$$
M_{t_{i}}(\omega)=\sum_{j=0}^{\infty} a_{j}^{t_{i}} L_{j}(\omega)
$$

Neste trabalho utiliza-se como funções básicas os polinômios de Laguerre ponderados: 


$$
\begin{gathered}
L_{0}(x)=e^{-x / 2}, \\
L_{1}(x)=e^{-x / 2}(1-x), \\
L_{2}(x)=e^{-x / 2}\left(1-2 x+0.5 x^{2}\right), \\
L_{n}(x)=e^{-x / 2} \frac{e^{x}}{n !} \frac{d^{n}\left(x^{n} e^{-x}\right)}{d x^{n}} .
\end{gathered}
$$

onde, em um modelo multifatorial, deve-se tomar produtos cruzados destas funções avaliados em cada uma das variáveis de estado.

A estimação de todos os coeficientes $a_{j}^{t_{i}}$ é impossível; contarna-se este problema definindo $M_{t_{i}}$ como sendo a projeção de $M_{t_{i}}$ no subespaço gerado pelas primeiras $N+1$ funções básicas, de modo que

$$
M_{t_{i}}^{N}(\omega)=\sum_{j=0}^{N} b_{j}^{t_{i}} L_{j}(\omega) .
$$

Os coeficientes $b_{j}^{t_{i}}$ podem ser estimados por mínimos quadrados da seguinte maneira. Seja $\left\{\omega_{z}\right\}_{z=1}^{Z}$ um conjunto de $Z$ trajetórias das variáveis de estado na medida neutra ao risco. Estima-se $M_{t_{K-1}}^{N}$ regredindo os ganhos realizados descontados pela taxa de juros $\left\{e^{r\left(t_{K}-t_{K-1}\right)} f\left(t_{K}, \omega_{z}\right)\right\}_{z=1}^{Z}$ contra as funções básicas avaliadas nas variáveis de estado. Utilizando a estimativa $\hat{M}_{t_{K-1}}^{N}$ pode-se calcular os ganhos realizados em $t_{K-1}$ para cada trajetória simulada como sendo o máximo entre o valor de continuação da opção e o valor de exercício. Mais precisamente, o ganho realizado será $\left\{\max \left(\hat{M}_{t_{K-1}}^{N}\left(\omega_{z}\right), f\left(t_{K-1}, \omega_{z}\right)\right)\right\}_{z=1}^{Z}$. O próximo passo é a estimação do valor de continuação em $t_{K-2}, M_{t_{K-2}}^{N}$, pela regressão dos ganhos realizados $\left\{e^{r\left(t_{K-1}-t_{K-2}\right)} \max \left(\hat{M}_{t_{K-1}}^{N}\left(\omega_{z}\right), f\left(t_{K-1}, \omega_{z}\right)\right)\right\}_{z=1}^{Z}$ contra as funções básicas avaliadas nas variáveis de estado em $t_{K-2}$ analogamente ao que foi feito na última regressão.

Proceda com estas regressões de maneira recursiva calculando $M_{t_{K-1}}^{N}, M_{t_{K-2}}^{N}, \ldots, M_{t_{1}}^{N}$. O valor da opção de bermuda será, aproximadamente, a média dos ganhos realizados em $t_{1}$ 
entre todas as trajetórias simuladas, isto é,

$$
\frac{1}{Z} \sum_{z=1}^{Z} \max \left(\hat{M}_{t_{1}}^{N}\left(\omega_{z}\right), f\left(t_{1}, \omega_{z}\right)\right)
$$

Utiliza-se esta média para aproximar o preço do derivtivo original. 


\section{7..3 Prova de que a função de verossimilhança pode ser ilimitada na presença de saltos}

Seja $z_{1}, z_{2}, \ldots, z_{N}$ uma amostra de uma mistura de duas distribuições normais

$$
p\left(z ; \omega, \mu_{1}, \mu_{2}, \sigma_{1}, \sigma_{2}\right)=\omega \phi\left(z ; \mu_{1}, \sigma_{1}^{2}\right)+(1-\omega) \phi\left(z ; \mu_{2}, \sigma_{2}^{2}\right),
$$

onde $\phi\left(z ; \mu, \sigma^{2}\right)$ é a densidade de $N\left(\mu, \sigma^{2}\right)$. A função de verossimilhança será

$$
l\left(\omega, \mu_{1}, \mu_{2}, \sigma_{1}, \sigma_{2} ; \mathbf{z}\right)=\sum_{i=1}^{N} \ln \left(p\left(z_{i} ; \omega, \mu_{1}, \mu_{2}, \sigma_{1}, \sigma_{2}\right)\right)
$$

com dominio no espaço paramétrico $\Theta \equiv[0,1] \times \mathbf{R} \times \mathbf{R} \times \mathbf{R}^{+} \times \mathbf{R}^{+} \subset \mathbf{R}^{5}$. É fácil ver que $\theta \equiv\left(0.5, z_{1}, 0,0,1\right)^{\prime} \in \bar{\Theta}$. Se $\left\{\theta_{n}\right\}_{n \geq 1}$ é uma sequencia com elementos em $\Theta$ que converge para $\theta$ entã

$$
p\left(z_{1} ; \theta_{n}\right) \rightarrow \infty
$$

e, para, $k \geq 2$,

$$
\begin{aligned}
\lim _{n \rightarrow \infty} p\left(z_{k} ; \theta_{n}\right) & =\underbrace{\frac{1}{2} \lim _{n \rightarrow \infty} \phi\left(z_{k} ; \operatorname{proj}_{2,4}\left(\theta_{n}\right)\right.}_{0})+\frac{1}{2} \lim _{n \rightarrow \infty} \phi\left(z_{k} ; \operatorname{proj}_{3,5}\left(\theta_{n}\right)\right) \\
& =\frac{1}{2 \sqrt{2 \pi}} e^{-\frac{1}{2} z_{k}^{2}}>0 .
\end{aligned}
$$

onde $\operatorname{proj}_{i, j}: \mathbf{R}^{5} \rightarrow \mathbf{R}^{2}$ é a função de projeção nas coordenadas $i$ e $j$. Isso prova que $p\left(z_{k} ; \theta_{n}\right)$ vai para o infinito se $k=1$ e vai para um número positivo se $k \geq 2$, de modo que $l\left(\theta_{n} ; \mathbf{z}\right) \rightarrow \infty$, como se queria demonstrar. 


\section{7..4 Especificação Bayesiana}

Ambos os modelos CYE e CYES serão estimados por meio de uma abordagem Bayesiana neste trabalho. O vetor de parâmetros a ser estimado no modelo com saltos é

$$
\Omega \equiv\left(\mu, \alpha, \kappa, \Sigma, \lambda_{\delta}, V, \phi, \bar{\eta}, \omega\right)
$$

O vetor de parâmetros do modelo sem saltos é o mesmo, excluindo-se as três últimas coordenadas $(\phi, \bar{\eta}, \omega)$, que correspondem a parâmetros de saltos.

Com o objetivo de tornar a distribuição a posteriori mais tratável, o vetor de parâmetros $\Omega$ será reparametrizado da seguinte maneira:

$$
\begin{gathered}
\mu_{m} \equiv \mu-\phi \bar{\eta}-\frac{1}{2} \sigma_{S}^{2} \\
\bar{\eta}_{m} \equiv \ln (1+\bar{\eta})-\frac{1}{2} \omega^{2} \\
\hat{\alpha} \equiv \alpha-\frac{\lambda_{\delta}}{\kappa}
\end{gathered}
$$

e o vetor de parâmetros a ser estimado torna-se

$$
\Theta \equiv\left(\mu_{m}, \alpha, \kappa, \Sigma, \hat{\alpha}, V, \phi, \bar{\eta}, \omega\right)
$$

Assume-se as distribuições a priori da tabela 14. 


\begin{tabular}{lccc}
\hline Parâmetro & Prior & Posteriori marginal & Modelo \\
\hline$\mu_{m}$ & $N\left(\mu_{0}, M_{0}\right)$ & $N(\cdot, \cdot)$ & CYE \& CYES \\
$\alpha$ & $N\left(\alpha_{0}, A_{0}\right)$ & $N(\cdot, \cdot)$ & CYE \& CYES \\
$\kappa$ & $N\left(\kappa_{0}, K_{0}\right)$ & Desconhecida & CYE \& CYES \\
$\Sigma^{-1}$ & $\mathbf{W}_{2}\left(\mathbf{R}_{0}, v_{0}\right)$ & Desconhecida & CYE \& CYES \\
$\hat{\alpha}$ & $N\left(\hat{\alpha}_{0}, G_{0}\right)$ & $N(\cdot, \cdot)$ & CYE \& CYES \\
$V^{-2}$ & $\Gamma\left(a_{V}, b_{V}\right)$ & $\Gamma(\cdot, \cdot)$ & CYE \& CYES \\
$\phi$ & $B(e, E)$ & $B(\cdot, \cdot)$ & CYES \\
$\bar{\eta}_{m}$ & $N\left(\mu_{\bar{\eta}}, \sigma_{\bar{\eta}}\right)$ & $N(\cdot, \cdot)$ & CYES \\
$\omega^{-2}$ & $\Gamma\left(a_{\omega}, b_{\omega}\right)$ & $\Gamma(\cdot, \cdot)$ & CYES \\
\hline
\end{tabular}

Tabela 14: Especificação das distribuições a posteriori nos modelos CYE e CYES 


\section{7..5 Dedução das distribuições a posteriori}

Esta seção é destinada a dedução de todas as distribuições a posteriori marginais utilizadas nos métodos de Gibbs-Sampling deste trabalho.

Aqui será conveniente tratar o modelo CYES como uma composição de quatro niveis hierárquicos: (1) a geração dos parâmetros segundo a distribuição de probabilidade $a$ priori; (2) a geração dos saltos dado os parâmetros de salto; (3) a geração do preço à vista e do convenience yield dados os parâmetros e as variáveis de salto; e (4) geração dos preços de contratos futuros dadas as variáveis latentes e os parâmetros. Mais precisamente,

$$
\begin{gathered}
\Theta \sim p(\Theta) \\
\Delta q_{i} \sim \operatorname{Bernoulli}(\Delta t \phi), \eta_{m i} \sim N\left(\bar{\eta}_{m}, \omega^{2}\right) \forall i=1, \ldots, N-1 \\
\xi_{i+1}=\mathbf{c}_{i}+\mathbf{Q} \xi_{i}+\varepsilon_{i}, \varepsilon_{i} \sim N(\mathbf{0}, \Sigma), \forall i=1, \ldots, N-1 \\
\mathbf{y}_{i}=\mathbf{d}+\mathbf{Z} \xi_{i}+\mathbf{v}_{i}, \mathbf{v}_{i} \sim N\left(\mathbf{0}, V^{2} \mathbf{I}_{K \times K}\right), \forall i=1, \ldots, N
\end{gathered}
$$

Para tornar as deduções mais simples faremos algumas convenções. Defina

$$
\begin{gathered}
\varepsilon_{X i}=X_{i+1}-X_{i}-\left(\mu_{m}-\delta\right) \Delta t-\eta_{m i} \Delta q_{i} \\
\varepsilon_{\delta i}=\delta_{i+1}-\delta_{i}-\kappa(\alpha-\delta) \Delta t
\end{gathered}
$$

e denote por $\hat{\mathbf{y}}\left(\xi_{i}, \Theta\right)$ o vetor de log-preços previstos utilizando-se os parâmetros $\Theta$. Dado o parâmetro $\theta \in \Theta$ utilizaremos a notação $\Theta_{-\theta}$ para denotar o vetor de parâmetros excluindo-se o parâmetro $\theta$.

\section{7..5.1 Dedução de $\boldsymbol{\mu}_{m} \mid \Theta_{-\mu_{m}}, \Delta \boldsymbol{q}, \boldsymbol{\eta}_{\boldsymbol{m}}, \boldsymbol{\xi}, \mathbf{y}$}

Tem-se $\mu_{m} \sim N\left(\mu_{0}, M_{0}\right)$. Logo, a distribuição a posteriori $p\left(\mu_{m} \mid \Theta_{-\mu_{m}}, \Delta q, \eta_{m}, \xi, \mathbf{y}\right)$ será proporcional a 
66

$$
\exp \left\{\frac{-\left(\mu_{m}-\mu_{0}\right)^{2}}{2 M_{0}}-\frac{1}{2\left(1-\rho^{2}\right)} \sum_{i=1}^{N-1}\left[\frac{\varepsilon_{X i}^{2}}{\Delta t \sigma_{S}^{2}}-2 \frac{\varepsilon_{X i} \varepsilon_{\delta i}}{\Delta t \sigma_{S} \sigma_{\delta}}\right]\right\}
$$

e a

$$
\begin{aligned}
& \exp \left\{-\frac{1}{2}\left[\frac{1}{M_{0}}+\frac{\Delta t(N-1)}{\left(1-\rho^{2}\right) \sigma_{S}^{2}}\right] \mu_{m}^{2}+\left[\frac{\mu_{0}}{M_{0}}\right.\right. \\
& \left.\left.+\quad \frac{\left.\sum\left(X_{i+1}-X_{i}+\delta_{i} \Delta t-\eta_{m i} \Delta q_{i}\right)\right)}{\sigma_{s}^{2}\left(1-\rho^{2}\right)}-\rho \frac{\sum\left(\delta_{i+1}-\delta_{i}-\kappa\left(\alpha-\delta_{i}\right) \Delta t\right.}{\sigma_{\delta} \sigma_{s}\left(1-\rho^{2}\right)}\right] \mu_{m}\right\}
\end{aligned}
$$

Definindo

$$
\begin{gathered}
\tilde{M}_{0}=\left[\frac{1}{M_{0}}+\frac{\Delta t(N-1)}{\left(1-\rho^{2}\right) \sigma_{S}^{2}}\right]^{-1} \\
\tilde{\mu}_{0}=\tilde{M}_{0}\left[\frac{\mu_{0}}{M_{0}}+\frac{\left.\sum\left(X_{i+1}-X_{i}+\delta_{i} \Delta t-\eta_{m i} \Delta q_{i}\right)\right)}{\sigma_{s}^{2}\left(1-\rho^{2}\right)}-\rho \frac{\sum\left(\delta_{i+1}-\delta_{i}-\kappa\left(\alpha-\delta_{i}\right) \Delta t\right.}{\sigma_{\delta} \sigma_{s}\left(1-\rho^{2}\right)}\right]
\end{gathered}
$$

verifica-se

$$
\mu_{m} \mid \Theta_{-\mu_{m}}, \Delta q, \eta_{m}, \xi, \mathbf{y} \sim N\left(\tilde{\mu}_{0}, \tilde{M}_{0}\right)
$$

\section{7..5.2 Dedução de $\alpha \mid \Theta_{-\alpha}, \Delta q, \eta_{m}, \xi, y$}

Tem-se $\alpha \sim N\left(\alpha_{0}, A_{0}\right)$. Logo, a distribuição a posteriori $p\left(\alpha \mid \Theta_{-\alpha}, \Delta q, \eta_{m}, \xi, \mathbf{y}\right)$ será proporcional a

$$
\exp \left\{\frac{-\left(\alpha-\alpha_{0}\right)^{2}}{2 A_{0}}-\frac{1}{2\left(1-\rho^{2}\right)} \sum_{i=1}^{N-1}\left[\frac{\varepsilon_{X i}^{2}}{\Delta t \sigma_{S}^{2}}-2 \frac{\varepsilon_{X i} \varepsilon_{\delta i}}{\Delta t \sigma_{S} \sigma_{\delta}}\right]\right\}
$$




$$
\begin{aligned}
& \exp \left\{-\frac{1}{2}\left[\frac{1}{A_{0}}+\frac{\kappa^{2} \Delta t(N-1)}{\left(1-\rho^{2}\right) \sigma_{\delta}^{2}}\right] \alpha^{2}+\left[\frac{\alpha_{0}}{A_{0}}+\kappa \frac{\left.\sum\left(\delta_{i+1}-\delta_{i}+\kappa \delta_{i} \Delta t\right)\right)}{\sigma_{\delta}^{2}\left(1-\rho^{2}\right)}\right.\right. \\
& \left.\left.-\quad \rho \kappa \frac{\sum\left(X_{i+1}-X_{i}-\left(\mu-\delta_{i}\right) \Delta t-\eta_{m i} \Delta q_{i}\right)}{\sigma_{\delta} \sigma_{s}\left(1-\rho^{2}\right)}\right] \mu_{m}\right\}
\end{aligned}
$$

Definindo

$$
\begin{gathered}
\tilde{A}_{0}=\left[\frac{1}{A_{0}}+\frac{\kappa^{2} \Delta t(N-1)}{\left(1-\rho^{2}\right) \sigma_{\delta}^{2}}\right]^{-1} \\
\tilde{\mu}_{0}=\tilde{A}_{0}\left[\frac{\alpha_{0}}{A_{0}}+\kappa \frac{\left.\sum\left(\delta_{i+1}-\delta_{i}+\kappa \delta_{i} \Delta t\right)\right)}{\sigma_{\delta}^{2}\left(1-\rho^{2}\right)}\right. \\
\left.-\rho \kappa \frac{\sum\left(X_{i+1}-X_{i}-\left(\mu-\delta_{i}\right) \Delta t-\eta_{m i} \Delta q_{i}\right)}{\sigma_{\delta} \sigma_{s}\left(1-\rho^{2}\right)}\right]
\end{gathered}
$$

tem-se

$$
\alpha \mid \Theta_{-\alpha}, \Delta q, \eta_{m}, \xi, \mathbf{y} \sim N\left(\tilde{\alpha}_{0}, \tilde{A}_{0}\right)
$$

\subsubsection{Dedução de $\hat{\alpha} \mid \Theta_{-\hat{\alpha}}, \Delta \boldsymbol{q}, \boldsymbol{\eta}_{\boldsymbol{m}}, \boldsymbol{\xi}, \mathbf{y}$}

Vale que $\hat{\alpha} \sim N\left(\hat{\alpha}_{0}, G_{0}\right)$. Logo, a distribuição a posteriori $p\left(\hat{\alpha} \mid \hat{\alpha}, \Delta q, \eta_{m}, \xi, \mathbf{y}\right)$ será proporcional a

$$
\exp \left\{\frac{-\left(\hat{\alpha}-\hat{\alpha}_{0}\right)^{2}}{2 G_{0}}-\frac{1}{V^{2}} \sum_{i=1}^{N} \sum_{j=1}^{K}\left(y_{i}^{j}-\hat{y^{j}}\left(\xi_{i}, \Theta_{-\hat{\alpha}}, \hat{\alpha}\right)\right)^{2}\right\}
$$




$$
\begin{aligned}
& \exp \left\{\frac{-\left(\hat{\alpha}-\hat{\alpha}_{0}\right)^{2}}{2 G_{0}}-\frac{1}{V^{2}} \sum_{i=1}^{N} \sum_{j=1}^{K}\left[y_{i}^{j}-\left(\frac{1-e^{-\kappa T_{j}}}{\kappa}-T_{j}\right) \hat{\alpha}\right.\right. \\
& -\quad X_{i}+\delta_{i}\left(\frac{1-e^{-\kappa T_{j}}}{\kappa}\right)-\left(r-\frac{1}{2} \frac{\sigma_{\delta}^{2}}{\kappa^{2}}-\frac{\rho \sigma_{S} \sigma_{\delta}}{\kappa}\right) T_{j} \\
& \left.\left.-\quad \frac{1}{4} \sigma_{\delta}^{2} \frac{1-e^{-2 \kappa T_{j}}}{\kappa^{3}}-\left(\rho \sigma_{S} \sigma_{\delta}-\frac{\sigma_{\delta}^{2}}{\kappa}\right)\left(\frac{1-e^{\kappa T_{j}}}{\kappa^{2}}\right)\right]^{2}\right\}
\end{aligned}
$$

Um simples argumento de completamento de quadrados mostra que

$$
\begin{aligned}
\tilde{G}_{0}=\left[\frac{1}{G_{0}}+\frac{N}{V^{2}} \sum_{j=1}^{K}\left(\frac{1-e^{-\kappa T_{j}}}{\kappa}-T_{j}\right)^{2}\right]^{-1} \\
{\hat{\alpha_{0}}}^{*}=\tilde{G}_{0}\left[\frac{\hat{\alpha}_{0}}{G_{0}}+\frac{1}{V^{2}} \sum_{i=1}^{N} \sum_{j=1}^{K}\left(\frac{1-e^{-\kappa T_{j}}}{\kappa}-T_{j}\right)\right. \\
\times\left(y_{i}^{j}-X_{i}+\delta_{i}\left(\frac{1-e^{-\kappa T_{j}}}{\kappa}\right)-\left(r-\frac{1}{2} \frac{\sigma_{\delta}^{2}}{\kappa^{2}}-\frac{\rho \sigma_{S} \sigma_{\delta}}{\kappa}\right) T_{j}\right. \\
\left.\left.-\frac{1}{4} \sigma_{\delta}^{2} \frac{1-e^{-2 \kappa T_{j}}}{\kappa^{3}}-\left(\rho \sigma_{S} \sigma_{\delta}-\frac{\sigma_{\delta}^{2}}{\kappa}\right)\left(\frac{1-e^{\kappa T_{j}}}{\kappa^{2}}\right)\right)\right]
\end{aligned}
$$

então

$$
\hat{\alpha}_{0} \mid \Theta_{-\hat{\alpha_{0}}}, \Delta q, \eta_{m}, \xi, \mathbf{y} \sim N\left({\hat{\alpha_{0}}}^{*}, \tilde{G}_{0}\right)
$$

\section{7..5.4 Dedução de $\boldsymbol{V}^{-2} \mid \Theta_{-V}, \Delta q, \eta_{m}, \boldsymbol{\xi}, \mathbf{y}$}

Tem-se $V^{-2} \sim \Gamma(a, b)$. Logo, $p\left(V^{-2} \mid \Theta_{-V}, \Delta q, \eta_{m}, \xi, \mathbf{y}\right)$ será proporcional a

$$
V^{-2(a-1)} e^{-b V^{2}} V^{-N K} \exp \left\{-\frac{1}{2 V^{2}} \sum_{i=1}^{N} \sum_{j=1}^{K}\left(y_{i}^{j}-\hat{y^{j}}\left(\xi_{i}, \Theta_{-V}\right)\right)^{2}\right\}
$$




$$
V^{-2\left(a+\frac{N K}{2}-1\right)} \exp \left\{-\frac{1}{2}\left[2 b+\sum_{i=1}^{N} \sum_{j=1}^{K}\left(y_{i}^{j}-\hat{y}^{j}\left(\xi_{i}, \Theta_{-V}\right)\right)^{2}\right]\right\}
$$

de modo que

$$
V^{-2} \mid \Theta_{-V}, \Delta q, \eta_{m}, \xi, \mathbf{y} \sim \Gamma\left[a+\frac{N}{2}, b+\frac{\sum_{i=1}^{N} \sum_{j=1}^{K}\left(y_{i}^{j}-\hat{y}^{j}\left(\xi_{i}, \Theta_{-V}\right)\right)^{2}}{2}\right]
$$

\section{7..5.5 Dedução de $\bar{\eta}_{m} \mid \Theta_{-\bar{\eta}_{m}}, \Delta \boldsymbol{q}, \boldsymbol{\eta}_{m}, \boldsymbol{\xi}, \mathbf{y}$}

Como $\eta_{m i} \sim \operatorname{iidN}\left(\bar{\eta}_{m}, \omega^{2}\right)$ deve-se ter

$$
\begin{aligned}
p\left(\bar{\eta}_{m} \mid \Theta_{-\bar{\eta}_{m}}, \Delta q, \eta_{m}, \xi, \mathbf{y}\right) & \propto \exp \left\{-\frac{\left(\bar{\eta}_{m}-\mu_{\bar{\eta}_{m}}\right)^{2}}{2 \sigma_{\bar{\eta}_{m}}^{2}}-\frac{1}{2 \omega^{2}} \sum_{i=1}^{N-1}\left(\eta_{i m}-\bar{\eta}_{m}\right)^{2}\right\} \\
& \propto \exp \left\{-\frac{1}{2}\left[\frac{1}{\sigma_{\bar{\eta}_{m}}^{2}}+\frac{N-1}{\omega^{2}}\right] \bar{\eta}_{m}^{2}\right\} \\
& \times \exp \left\{\left[\frac{\mu_{\bar{\eta}_{m}}}{\sigma_{\bar{\eta}_{m}}^{2}}+\frac{\sum_{i=1}^{N-1} \eta_{m i}}{\omega^{2}}\right] \bar{\eta}_{m}\right\}
\end{aligned}
$$

Logo, $\bar{\eta}_{m} \mid \Theta_{-\bar{\eta}_{m}}, \Delta q, \eta_{m}, \xi, \mathbf{y}$ seguirá a distribuição

$$
N\left(\left[\frac{1}{\sigma_{\bar{\eta}_{m}}^{2}}+\frac{N-1}{\omega^{2}}\right]^{-1}\left[\frac{\mu_{\bar{\eta}_{m}}}{\sigma_{\bar{\eta}_{m}}^{2}}+\frac{\sum_{i=1}^{N-1} \eta_{m i}}{\omega^{2}}\right],\left[\frac{1}{\sigma_{\bar{\eta}_{m}}^{2}}+\frac{N-1}{\omega^{2}}\right]^{-1}\right)
$$

\section{7..5.6 Dedução de $\omega^{-2} \mid \Theta_{\omega}, \Delta q, \eta_{m}, \xi, y$}

Tem-se $\omega^{-2} \sim \Gamma\left(a_{\omega}, b_{\omega}\right)$. Logo, $p\left(\omega^{-2} \mid \Theta_{-\omega}, \Delta q, \eta_{m}, \xi, \mathbf{y}\right)$ será proporcional a

$$
\omega^{-2\left(a_{\omega}-1\right)} e^{-b_{\omega} \omega^{2}} \omega^{-N-1} \exp \left\{-\frac{1}{2 \omega^{2}} \sum_{i=1}^{N-1}\left(\eta_{m i}-\bar{\eta}_{m}\right)^{2}\right\}
$$




$$
\left.\omega^{-2\left(a_{\omega}+\frac{N-1}{2}-1\right)} \exp \left\{-\frac{1}{2}\left[2 b_{\omega}+\sum_{i=1}^{N-1}\left(\eta_{m i}-\bar{\eta}_{m}\right)^{2}\right\}\right]\right\}
$$

de modo que

$$
\omega^{-2} \mid \Theta_{-\omega}, \Delta q, \eta_{m}, \xi, \mathbf{y} \sim \Gamma\left[a_{\omega}+\frac{N-1}{2}, b_{\omega}+\frac{\sum_{i=1}^{N-1}\left(\eta_{m i}-\bar{\eta}_{m}\right)^{2}}{2}\right]
$$

\section{7..5.7 Dedução de $\phi \mid \Theta_{\phi}, \Delta \boldsymbol{q}, \boldsymbol{\eta}_{\boldsymbol{m}}, \boldsymbol{\xi}, \mathbf{y}$}

Tem-se $\Delta t \phi \sim \mathbf{B}(e, E)$. Logo,

$$
\begin{aligned}
p\left(\Delta t \phi \mid \Theta_{-\phi}, \Delta q, \eta_{m}, \xi, \mathbf{y}\right) & \propto\left[(\Delta t \phi)^{\sum \Delta q_{i}}(1-\Delta t \phi)^{N-1-\sum \Delta q_{i}}\right] \\
& \times(\Delta t \phi)^{e-1}(1-\Delta t \phi)^{E-1} \\
& \propto(\Delta t \phi)^{\sum \Delta q_{i} e-1}(1-\Delta t \phi)^{N-1-\sum \Delta q_{i}+E-1}
\end{aligned}
$$

de modo que

$$
\Delta t \phi \mid \Theta_{-\phi}, \Delta q, \eta_{m}, \xi, \mathbf{y} \sim \mathbf{B}\left(\Delta q_{i} e, N-1-\sum \Delta q_{i}+E\right)
$$

\section{7..5.8 Dedução de $\Delta \boldsymbol{q}_{i} \mid \Theta,\left\{\Delta \boldsymbol{q}_{j}\right\}_{j \neq i}, \eta_{m}, \boldsymbol{\xi}, \mathbf{y}$}

$\Delta q_{i} \mid \Theta,\left\{\Delta q_{j}\right\}_{j \neq i}, \eta_{m}, \xi, \mathbf{y}$ pode assumir valores apenas no conjunto $\{0,1\}$. Logo, sua distribuição é do tipo Bernoulli. Segue-se que

$$
P\left(\Delta q_{i}=1 \mid \Theta,\left\{\Delta q_{j}\right\}_{j \neq i}, \eta_{m}, \xi, \mathbf{y}\right) \propto \Delta t \phi p\left(\xi, \mathbf{y} \mid \Theta,\left\{\Delta q_{j}\right\}_{j \neq i}, \eta_{m}, \Delta q_{i}=1\right)
$$

$$
P\left(\Delta q_{i}=0 \mid \Theta,\left\{\Delta q_{j}\right\}_{j \neq i}, \eta_{m}, \xi, \mathbf{y}\right) \propto(1-\Delta t \phi) p\left(\xi, \mathbf{y} \mid \Theta,\left\{\Delta q_{j}\right\}_{j \neq i}, \eta_{m}, \Delta q_{i}=0\right)
$$


Logo, definindo

$$
\begin{aligned}
C_{1} & =\Delta t \phi \exp \left\{-\frac{1}{2\left(1-\rho^{2}\right)}\left[\frac{\left(X_{i+1}-X_{i}-\left(\mu_{m}-\delta_{i}\right) \Delta t-\eta_{m i}\right)^{2}}{\Delta t \sigma_{S}^{2}}\right.\right. \\
& \left.\left.-2 \rho \frac{\left(X_{i+1}-X_{i}-\left(\mu_{m}-\delta_{i}\right) \Delta t-\eta_{m i}\right)\left(\delta_{i+1}-\delta_{i}-\left(\alpha-\delta_{i}\right) \Delta t\right)}{\Delta t \sigma_{S} \sigma_{\delta}}\right]\right\} \\
C_{2} & =(1-\Delta t \phi) \exp \left\{-\frac{1}{2\left(1-\rho^{2}\right)}\left[\frac{\left(X_{i+1}-X_{i}-\left(\mu_{m}-\delta_{i}\right) \Delta t\right)^{2}}{\Delta t \sigma_{S}^{2}}\right.\right. \\
& \left.\left.-2 \rho \frac{\left(X_{i+1}-X_{i}-\left(\mu_{m}-\delta_{i}\right) \Delta t\right)\left(\delta_{i+1}-\delta_{i}-\left(\alpha-\delta_{i}\right) \Delta t\right)}{\Delta t \sigma_{S} \sigma_{\delta}}\right]\right\}
\end{aligned}
$$

tem-se

$$
\begin{aligned}
& P\left(\Delta q_{i}=1 \mid \Theta,\left\{\Delta q_{j}\right\}_{j \neq i}, \eta_{m}, \xi, \mathbf{y}\right)=\frac{C_{1}}{C_{1}+C_{2}}, \\
& P\left(\Delta q_{i}=0 \mid \Theta,\left\{\Delta q_{j}\right\}_{j \neq i}, \eta_{m}, \xi, \mathbf{y}\right)=\frac{C_{2}}{C_{1}+C_{2}} .
\end{aligned}
$$

\section{7..5.9 Dedução de $\boldsymbol{\eta}_{m i} \mid \Theta, \Delta \boldsymbol{q},\left\{\boldsymbol{\eta}_{\boldsymbol{m} j}\right\}_{j \neq i}, \boldsymbol{\xi}, \mathbf{y}$}

Tem-se

$$
p\left(\eta_{m i} \mid \Theta, \Delta q,\left\{\eta_{m j}\right\}_{j \neq i}, \xi, \mathbf{y}\right) \propto p\left(\eta_{m i} \mid \Theta\right) p\left(\xi, \mathbf{y} \mid \Theta, \Delta q,\left\{\eta_{m j}\right\}_{j \neq i}, \eta_{m i}\right)
$$

Logo, $p\left(\eta_{m i} \mid \Theta, \Delta q,\left\{\eta_{m j}\right\}_{j \neq i}, \xi, \mathbf{y}\right)$ será proporcional a 


$$
\begin{aligned}
& \exp \left\{-\frac{\left(\eta_{m i}-\bar{\eta}_{m}\right)^{2}}{2 \omega^{2}}-\frac{1}{2\left(1-\rho^{2}\right)}\left[\frac{\varepsilon_{X i}^{2}}{\Delta t \sigma_{s}^{2}}-2 \rho \frac{\varepsilon_{X i} \varepsilon_{\delta i}}{\Delta t \sigma_{s} \sigma_{\delta}}\right]\right\} \\
\propto & \exp \left\{-\frac{1}{2}\left[\frac{1}{2 \omega^{2}}+\frac{\Delta q_{i}}{\left(1-\rho^{2}\right) \Delta t \sigma_{S}^{2}}\right] \eta_{m i}^{2}\right. \\
+ & {\left[\frac{\bar{\eta}_{m}}{\omega^{2}}+\frac{\left(X_{i+1}-X_{i}-\left(\mu_{m}-\delta_{i}\right) \Delta t\right) \Delta q_{i}}{\left(1-\rho^{2}\right) \delta_{S}^{2} \Delta t}\right.} \\
- & \left.\left.\rho \frac{\left(\delta_{i+1}-\delta_{i}-\kappa\left(\alpha-\delta_{i}\right) \Delta t\right) \Delta q_{i}}{\left(1-\rho^{2}\right) \Delta t \sigma_{s} \sigma_{\delta}}\right] \eta_{m i}\right\} .
\end{aligned}
$$

Defina

$$
\begin{gathered}
\sigma_{i}^{2}=\left[\frac{1}{2 \omega^{2}}+\frac{\Delta q_{i}}{\left(1-\rho^{2}\right) \Delta t \sigma_{S}^{2}}\right]^{-1} \\
\mu_{i}=\sigma_{i}^{2}\left[\frac{\bar{\eta}_{m}}{\omega^{2}}+\frac{\left(X_{i+1}-X_{i}-\left(\mu_{m}-\delta_{i}\right) \Delta t\right) \Delta q_{i}}{\left(1-\rho^{2}\right) \delta_{S}^{2} \Delta t}-\rho \frac{\left(\delta_{i+1}-\delta_{i}-\kappa\left(\alpha-\delta_{i}\right) \Delta t\right) \Delta q_{i}}{\left(1-\rho^{2}\right) \Delta t \sigma_{s} \sigma_{\delta}}\right] .
\end{gathered}
$$

Então

$$
\eta_{m i} \mid \Theta, \Delta q,\left\{\eta_{m j}\right\}_{j \neq i}, \xi, \mathbf{y} \sim N\left(\mu_{i}, \sigma_{i}^{2}\right)
$$




\section{7..6 Descrição do algoritmo MCMC}

Nesta seção faz-se uma precisa descrição do algoritmo MCMC utilizado para estimar os modelos CYES e CYE.

O objetivo é simular uma cadeia cuja distribuição estacionária é dada por

$$
p\left(\Theta,\left\{\xi_{i}\right\}_{i=1}^{N},\left\{\Delta q_{i}\right\}_{i=1}^{N-1},\left\{\eta_{i}\right\}_{i=1}^{N-1} \mid \mathbf{y}\right)
$$

A distribuição 7.139 será simulada por meio de um algoritmo MCMC composto por uma mistura de passos do tipo Gibbs-Sampling e de passos do tipo Metropolos-Hastings genérico.

Os parâmetros $\mu_{m}, \alpha, \hat{\alpha}, V^{-2}, \phi, \bar{\eta}_{m}, \omega^{-2}$ possuem distribuição a posteriori conhecida e serão simulados por Gibbs-Sampling. $\Sigma$ possui uma distribuição a posteriori desconhecida, mas sua distribuição condicional apenas às variáveis de estado é Wishart e será utilizada como densidade geradora de candidatos em seu passo do algoritmo. $\kappa$ será simulado por um passeio aleatorio. Finalmente, as variáveis de estado $\left\{\xi_{i}\right\}_{i=1}^{N}$ serão simuladas pelo método de Jong \& Shephard (1995)

\section{7..6.1 Simulação de $\Sigma$}

A distribuição de $\Sigma$ condicionalmente aos outros parâmetros e às variáveis de estado é

$$
\begin{aligned}
p\left(\Sigma \mid \Theta_{-\Sigma}, \Delta q, \eta_{m}, \xi\right) & \propto \frac{|\Sigma|^{\frac{v_{0}-3}{2}}}{\mathbf{R}_{0}^{\frac{v_{0}}{2}}} \exp \left\{-\frac{1}{2} \operatorname{Tr}\left(\mathbf{R}_{0}^{-1} \Sigma\right)\right\} \\
& \times|\Sigma|^{\frac{N}{2}} \exp \left\{-\frac{1}{2} \sum_{i=1}^{N-1} \mathbf{r}_{i}^{\top} \Sigma \mathbf{r}_{i}\right\} \\
& \propto|\Sigma|^{\frac{v_{0}+N-4}{2}} \exp \left\{-\frac{1}{2}\left[\operatorname{Tr}\left(\mathbf{R}_{0}^{-1} \Sigma\right)+\sum_{i=1}^{N-1} \operatorname{Tr}\left(\mathbf{r}_{i} \mathbf{r}_{i}^{\top} \Sigma\right)\right]\right\} \\
& \propto|\Sigma|^{\frac{v_{0}+N-4}{2}} \exp \left\{-\frac{1}{2}\left[\operatorname{Tr}\left(\mathbf{R}_{0}^{-1} \Sigma+\sum_{i=1}^{N-1} \mathbf{r}_{i} \mathbf{r}_{i}^{\top} \Sigma\right)\right]\right\}
\end{aligned}
$$


onde $\mathbf{r}_{i}=\xi_{i+1}-\mathbf{c}_{i}-\mathbf{Q} \xi_{i}$. Logo, definindo

$$
v_{n}=v_{0}+N-1 \text { and } \mathbf{R}_{n}=\left(\mathbf{R}_{0}^{-1}+\sum_{i=1}^{N-1} \mathbf{r}_{i} \mathbf{r}_{i}^{\top}\right)^{-1}
$$

segue-se que

$$
\Sigma \mid \Theta_{-\Sigma}, \Delta q, \eta_{m}, \xi \sim \mathbf{W}_{2}\left(v_{n}, \mathbf{R}_{n}\right)
$$

Suponha agora que todos os outros parâmetros e variáveis de estado estão fixados e que se deseja amostrar $\Sigma$ via Metropolis-Hastings utilizando-se 7.145 como densidade geradora de candidatos. A probabilidade de aceitar um candidato $\Sigma_{c}$ dado um valor precedente $\Sigma$ será

$$
\frac{p\left(\Sigma_{c} \mid \Theta_{-\Sigma}, \Delta q, \eta_{m}, \xi, \mathbf{y}\right)}{p\left(\Sigma \mid \Theta_{-\Sigma}, \Delta q, \eta_{m}, \xi, \mathbf{y}\right)} \frac{p\left(\Sigma \mid \Theta_{-\Sigma}, \Delta q, \eta_{m}, \xi\right)}{p\left(\Sigma_{c} \mid \Theta_{-\Sigma}, \Delta q, \eta_{m}, \xi\right)} \frac{p\left(\mathbf{y} \mid \Sigma_{c}, \Theta_{-\Sigma}, \Delta q, \eta_{m}, \xi\right)}{p\left(\mathbf{y} \mid \Sigma, \Theta_{-\Sigma}, \Delta q, \eta_{m}, \xi\right)}
$$

Que é igual a

$$
\exp \left\{-\frac{1}{2 V^{2}}\left[\sum\left(y_{i}^{j}-\hat{y^{j}}\left(\xi_{i}, \Sigma_{c}\right)\right)^{2}-\sum\left(y_{i}^{j}-\hat{y^{j}}\left(\xi_{i}, \Sigma\right)\right)^{2}\right]\right\}
$$

Em outras palavras, a probabilidade de aceitar o candidato será função da mudança no erro quadrático médio.

\section{7..6.2 Simulação de $\boldsymbol{\kappa}$}

Para a simulação de $\kappa$ utilizaremos um processo de passeio aleatorio conhecido como MALA. dado um valor precedente, $\kappa$, um novo candidato $\kappa_{c}$ será gerado de acordo com a seguinte densidade:

$$
\kappa_{c} \sim N\left(\kappa+\frac{\sigma_{\kappa}^{2}}{2} \frac{\partial p\left(\kappa \mid \Theta_{-\kappa}, \Delta q, \eta_{m}, \xi, \mathbf{y}\right)}{\partial \kappa}, \sigma_{\kappa}^{2}\right)
$$


onde $\sigma_{\kappa}$ é escolhido de maneira apropriada para manter a autocorrelação da cadeia em niveis razoaveis. A probabilidade de aceitar o candidato será

$$
\frac{p\left(\kappa_{c} \mid \Theta_{-\kappa}, \Delta q, \eta_{m}, \xi, \mathbf{y}\right)}{p\left(\kappa \mid \Theta_{-\kappa}, \Delta q, \eta_{m}, \xi, \mathbf{y}\right)} \frac{\exp \left\{-\frac{1}{2 \sigma_{\kappa}}\left(\kappa-\kappa_{c}-\frac{\partial p\left(\kappa_{c} \mid \Theta_{-\kappa}, \Delta q, \eta_{m}, \xi, \mathbf{y}\right)}{\partial \kappa}\right)^{2}\right\}}{\exp \left\{-\frac{1}{2 \sigma_{\kappa}}\left(\kappa_{c}-\kappa-\frac{\partial p\left(\kappa \mid \Theta_{-\kappa}, \Delta q, \eta_{m}, \xi, \mathbf{y}\right)}{\partial \kappa}\right)^{2}\right\}} .
$$

\section{7..6.3 Simulação de $\left\{\xi_{i}\right\}_{i=i}^{N}$}

$\left\{\xi_{i}\right\}_{i=i}^{N} \mid \Theta, \Delta q, \eta_{m}, \mathbf{y}$ segue uma distribuição normal multivariada com média dada pelo smoothed Kalman Filter. Utiliza-se aqui o algoritmo de Jong \& Shephard (1995) para gerar amostragens desta distribuição. Defina

$$
\begin{gathered}
G=\left[V \mathbf{I}_{K \times K}, \mathbf{0}_{K \times 2}\right] \\
H=\left[\mathbf{0}_{2 \times K}, \Sigma^{\frac{1}{2}}\right]
\end{gathered}
$$

Sejam $a_{1}$ e $P_{1}$ os chutes iniciais para o primeiro valor da cadeia e para a matriz de erro quadrático médio de $a_{1}$, respectivamente. Rode, para $i=1, \ldots, N$,

$$
\begin{gathered}
e_{i}=y_{i}-\mathbf{d}-\mathbf{Z} a_{i} \\
\mathbf{D}_{i}=\mathbf{Z P}_{i} \mathbf{Z}^{\top}+\mathbf{G G}^{\top} \\
\mathbf{K}_{i}=\mathbf{Q P}_{i} \mathbf{Z}^{\top} \mathbf{D}_{i}^{-1} \\
a_{i+1}=\mathbf{c}+\mathbf{Q} a_{i}+\mathbf{K}_{i} e_{i}
\end{gathered}
$$




$$
P_{i+1}=\mathbf{Q} P_{i}\left(\mathbf{Q}-\mathbf{K}_{i} \mathbf{Z}\right)^{\top}+\mathbf{H} \mathbf{H}^{\top}
$$

e armazene $\left\{e_{i}\right\}_{i=1}^{N},\left\{\mathbf{D}_{i}\right\}_{i=1}^{N}$ e $\left\{\mathbf{K}_{i}\right\}_{i=1}^{N}$. Defina $r_{N}=0, U_{N}=0, \Omega=\mathbf{H H}^{\top}$ e rode, para $i=N, \ldots, 1$,

$$
\begin{gathered}
C_{i}=\Omega-\Omega U_{i} \Omega \\
\varepsilon_{i} \sim N\left(0, C_{i}\right) \\
\mathbf{M}_{i}=\Omega U_{i}\left(\mathbf{Q}-\mathbf{K}_{i} \mathbf{Z}\right) \\
r_{i-1}=\mathbf{Z}^{\top} \mathbf{D}_{i}^{-1} e_{i}+\left(\mathbf{Q}-\mathbf{K}_{i} \mathbf{Z}\right)^{\top} r_{i}+\mathbf{M}_{i}^{\top} C_{i}^{-1} \varepsilon_{i} \\
U_{i-1}=\mathbf{Z}^{\top} \mathbf{D}_{i}^{-1} \mathbf{Z}+\left(\mathbf{Q}-\mathbf{K}_{i} \mathbf{Z}\right)^{\top} U_{i}\left(\mathbf{Q}-\mathbf{K}_{i} \mathbf{Z}\right)+\mathbf{M}_{i}^{\top} C_{i}^{-1} \mathbf{M}_{i}
\end{gathered}
$$

e armazene $\left\{r_{i}\right\}_{i=1}^{N},\left\{\varepsilon_{i}\right\}_{i=1}^{N}$. Jong \& Shephard (1995) mostra que os erros das variáveis de estado condicionados aos valores das variáveis de medida podem ser simulados por

$$
\nu_{i}=\mathbf{Q} r_{i}+\varepsilon_{i} \text { for } i=1, \ldots, N
$$

Finalmente, $\left\{\xi_{i}\right\}_{i=i}^{N} \mid \Theta, \Delta q, \eta_{m}, \mathbf{y}$ pode ser simulado por

$$
\begin{gathered}
\hat{\xi}_{1}=a_{1}+\nu_{1} \\
\hat{\xi}_{i+1}=\mathbf{c}+\mathbf{Q} \hat{\xi}_{i}+\nu_{i+1} \text { for } i=1, \ldots, N-1 .
\end{gathered}
$$




\section{7..6.4 Algoritmo de MCMC completo}

Seja $M$ o número de elementos a ser gerados para a cadeia. O algoritmo de MCMC utilizado neste trabalho pode ser enunciado da seguinte maneira:

Faça, para $z=1, \ldots, M-1$

- Gere $\mu_{m}^{z+1} \sim \mu_{m} \mid \Theta_{-\mu_{m}}, \Delta q^{z}, \eta_{m}^{z}, \xi^{z}, \mathbf{y}$ utilizando 7.92 (Gibbs-Sampling);

- Gere $\alpha^{z+1} \sim \alpha \mid \Theta_{-\alpha}, \Delta q^{z}, \eta_{m}^{z}, \xi^{z}, \mathbf{y}$ utilizando 7.99 (Gibbs-Sampling);

- Gere $\kappa^{z+1}$ utilizando 7.148 como densidade geradora de candidatos e 7.149 como probabilidade de aceite (Metropolis-Hastings MALA);

- gere $\Sigma^{z+1}$ usando 7.145 como densidade geradora de candidatos e 7.147 como probabilidade de aceite (Metropolis-Hastings);

- Gere $\hat{\alpha}^{z+1} \sim \hat{\alpha} \mid \Theta_{-\hat{\alpha}}, \Delta q^{z}, \eta_{m}^{z}, \xi^{z}, \mathbf{y}$ utilizando 7.108 (Gibbs-Sampling);

- Gere $\left(V^{-2}\right)^{z+1} \sim V^{-2} \mid \Theta_{-V}, \Delta q^{z}, \eta_{m}^{z}, \xi^{z}, \mathbf{y}$ utilizando 7.111 (Gibbs-Sampling);

- Gere $\bar{\eta}_{m}^{z+1} \sim \bar{\eta}_{m} \mid \Theta_{-\bar{\eta}_{m}}, \Delta q^{z}, \eta_{m}^{z}, \xi^{z}, \mathbf{y}$ utilizando 7.115 (Gibbs-Sampling);

- Gere $\left(\omega^{-2}\right)^{z+1} \sim \omega^{-2} \mid \Theta_{-\omega}, \Delta q^{z}, \eta_{m}^{z}, \xi^{z}, \mathbf{y}$ utilizando 7.118 (Gibbs-Sampling);

- Gere $\phi^{z+1} \sim \phi \mid \Theta_{-\phi}, \Delta q^{z}, \eta_{m}^{z}, \xi^{z}, \mathbf{y}$ utilizando 7.122 (Gibbs-Sampling);

- Faça, para $i=1 \ldots, N-1$

Gere $\eta_{m i}^{z+1} \sim \eta_{m i} \mid \Theta, \Delta q^{z},\left\{\eta_{m j}\right\}_{j \neq i}, \xi^{z}, \mathbf{y}$ utilizando 7.138 (GibbsSampling);

- Faça, para $i=1 \ldots, N-1$

Gere $\Delta q_{i}^{z+1} \sim \Delta q_{i} \mid \Theta,\left\{\Delta q_{j}\right\}_{j \neq i}, \eta_{m}, \xi, \mathbf{y}$ utilizando 7.129 (Gibbs-

Sampling);

- Gere $\left\{\xi_{i}^{z+1}\right\}_{i=i}^{N} \sim\left\{\xi_{i}\right\}_{i=i}^{N} \mid \Theta, \Delta q^{z+1}, \eta_{m}^{z+1}, \mathbf{y}$ utilizando o método 7..6.3 (Gibbs-Sampling); 
7..7 Exemplo das cadeias de Markov: modelo CYES e dados de cobre
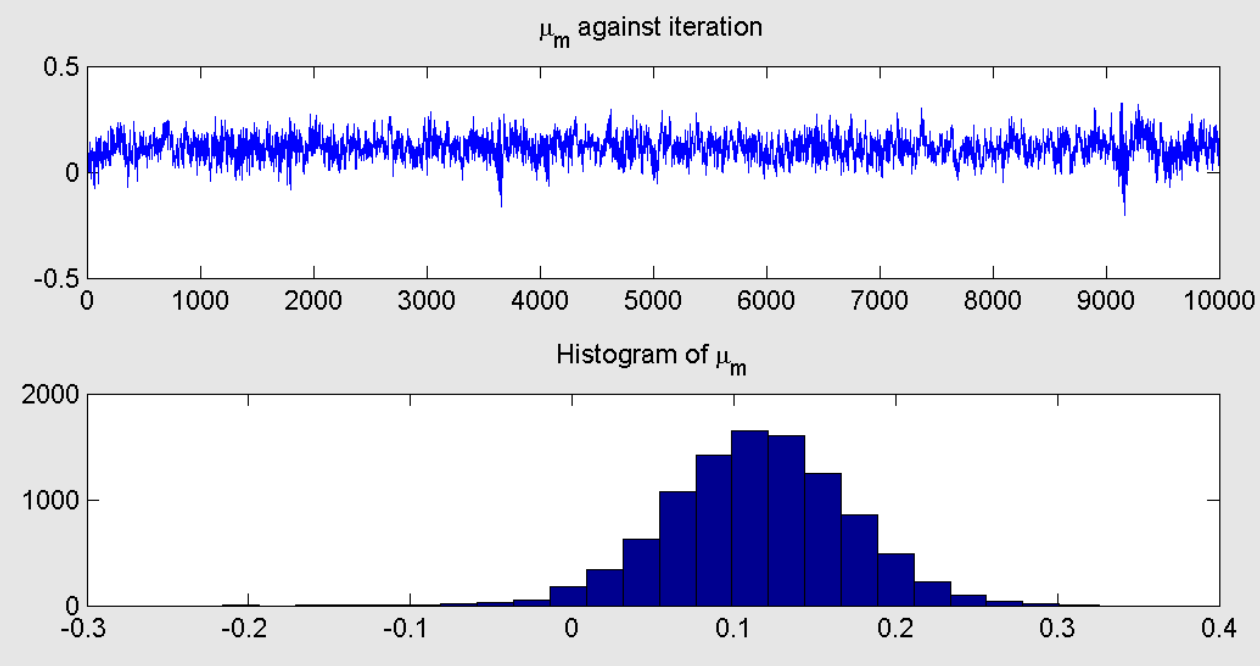

Correlanogram of $\mu_{\mathrm{m}}$

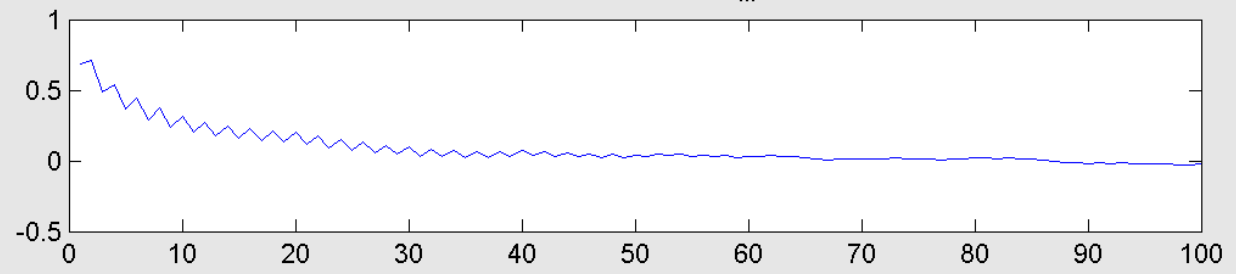

Figura 12: Simulação de $\boldsymbol{\mu}_{m} \mid \mathrm{y}$ utilizando o modelo CYES e dados de cobre O gráfico superior exibe a cadeia simulada para o parâmetro. O gráfico intermediario é o histograma dos valores simulados. O gráfico inferior é o correlograma da cadeia simulada. 

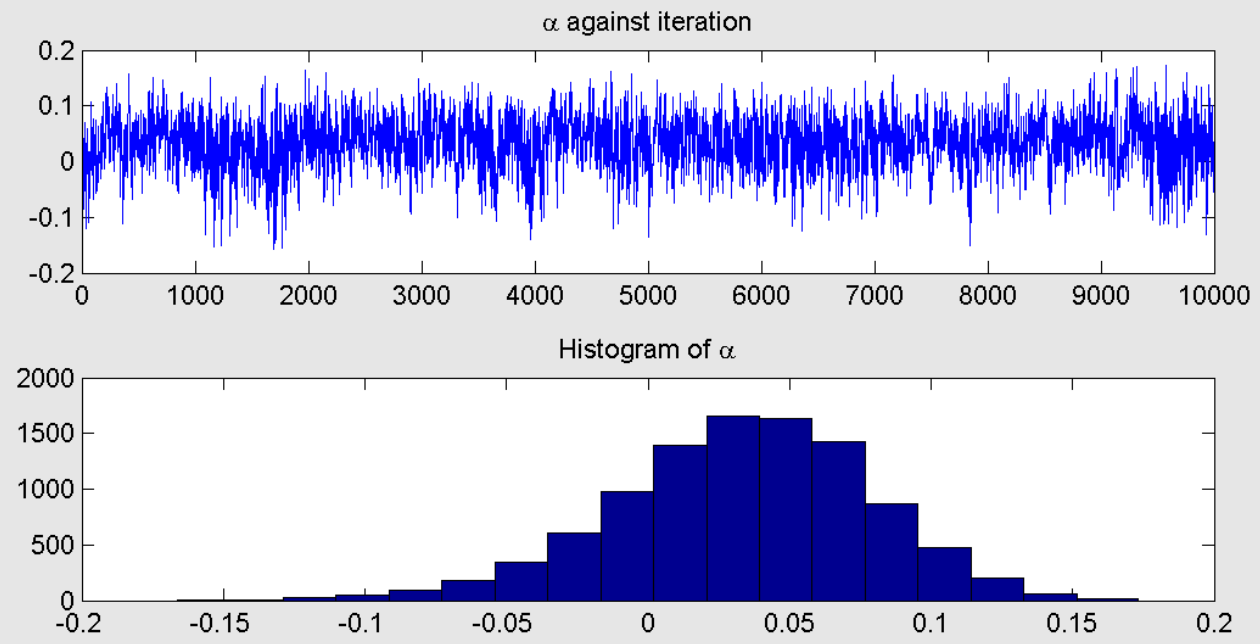

Correlanogram of $\alpha$

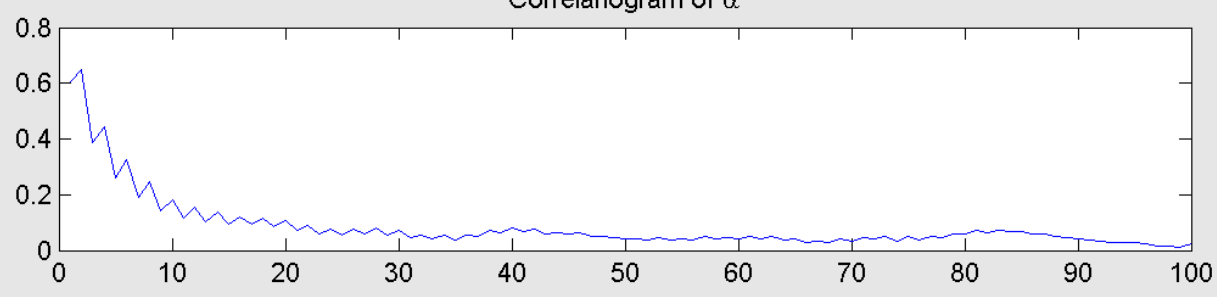

Figura 13: Simulação de $\boldsymbol{\alpha} \mid$ y utilizando o modelo CYES e dados de cobre

O gráfico superior exibe a cadeia simulada para o parâmetro. O gráfico intermediario é o histograma dos valores simulados. O gráfico inferior é o correlograma da cadeia simulada.
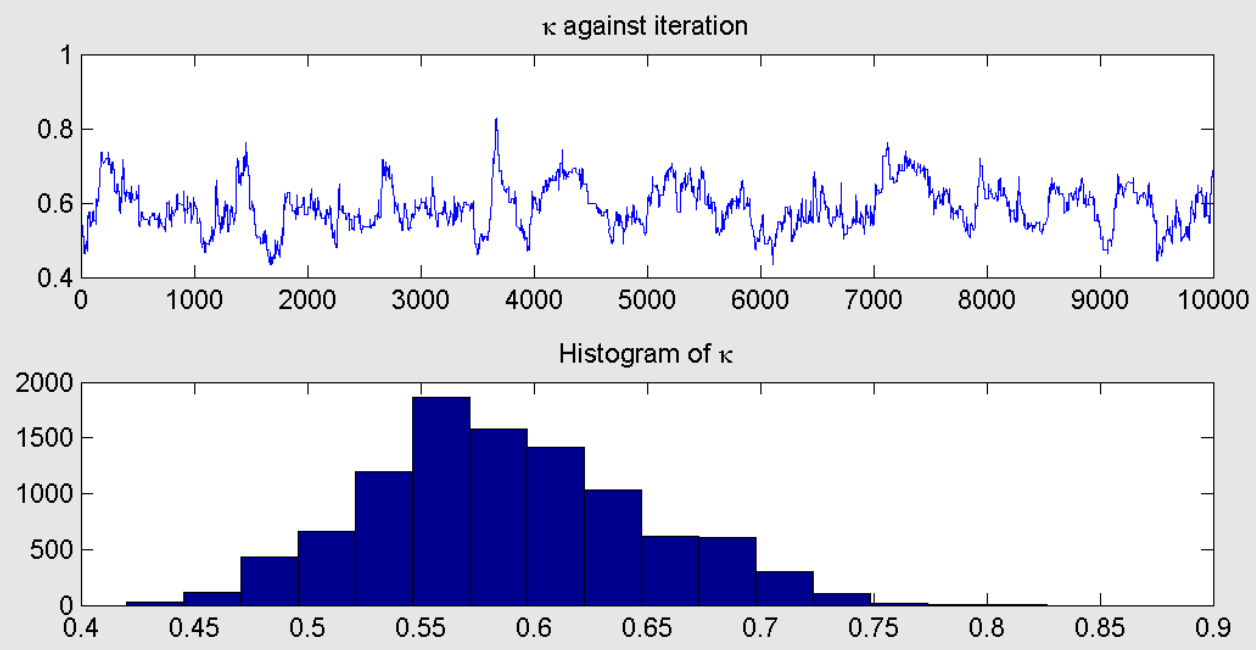

Correlanogram of $\kappa$

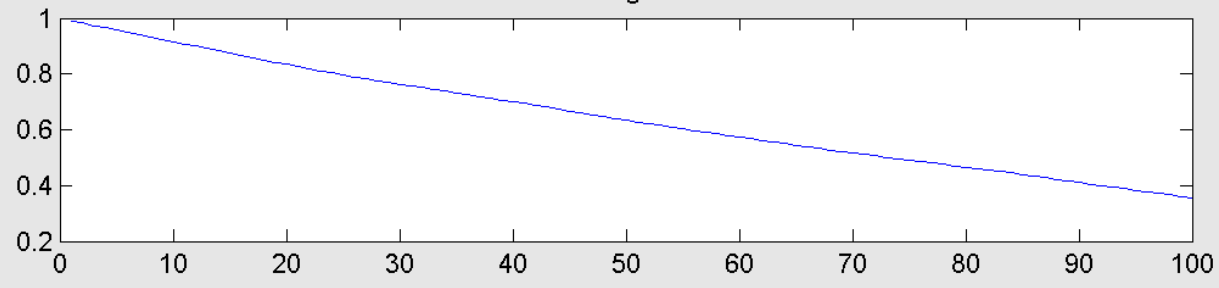

Figura 14: Simulação de $\kappa \mid$ y utilizando o modelo CYES e dados de cobre

O gráfico superior exibe a cadeia simulada para o parâmetro. O gráfico intermediario é o histograma dos valores simulados. O gráfico inferior é o correlograma da cadeia simulada. 

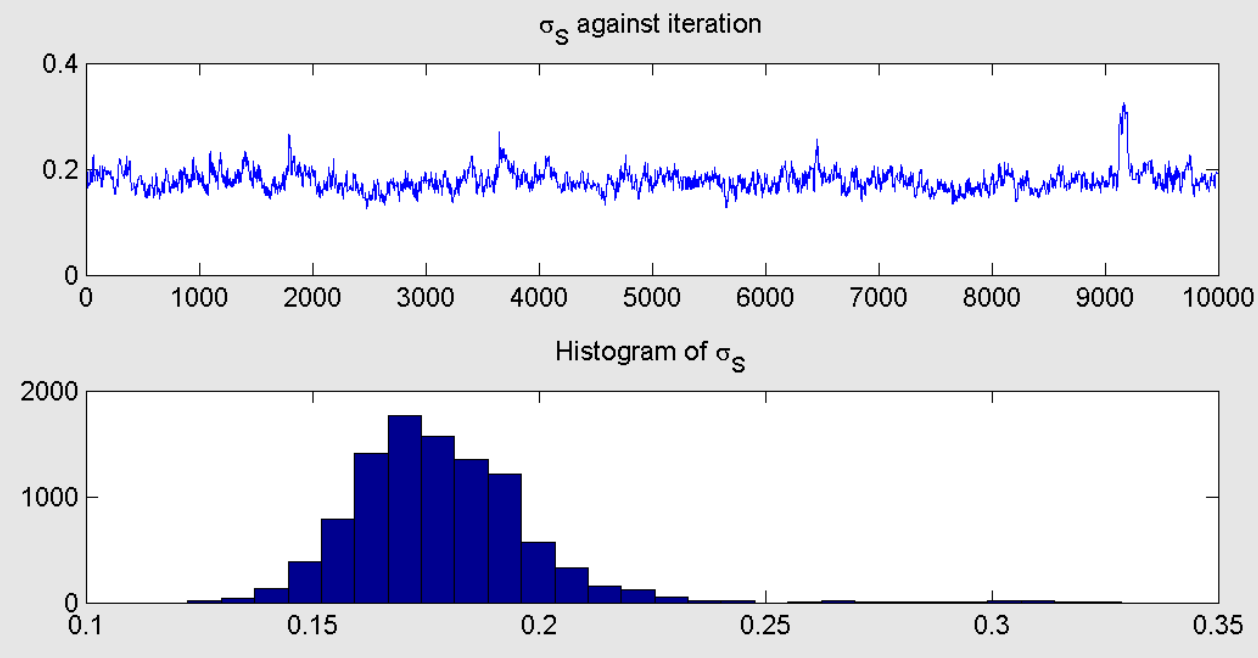

Correlanogram of $\sigma_{\mathrm{S}}$

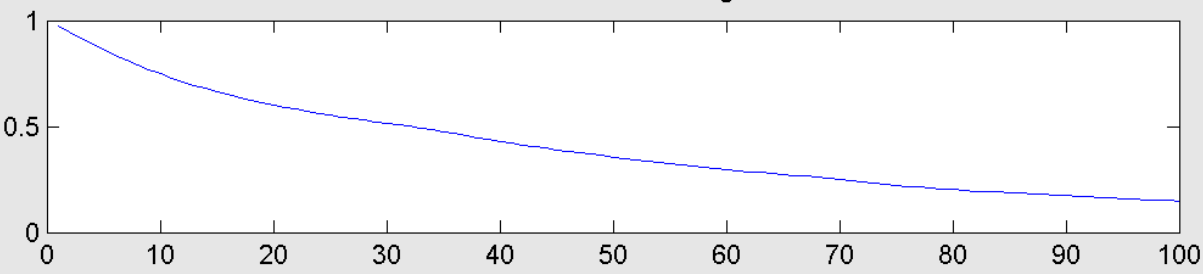

Figura 15: Simulação de $\sigma_{S} \mid$ y utilizando o modelo CYES e dados de cobre O gráfico superior exibe a cadeia simulada para o parâmetro. O gráfico intermediario é o histograma dos valores simulados. O gráfico inferior é o correlograma da cadeia simulada.
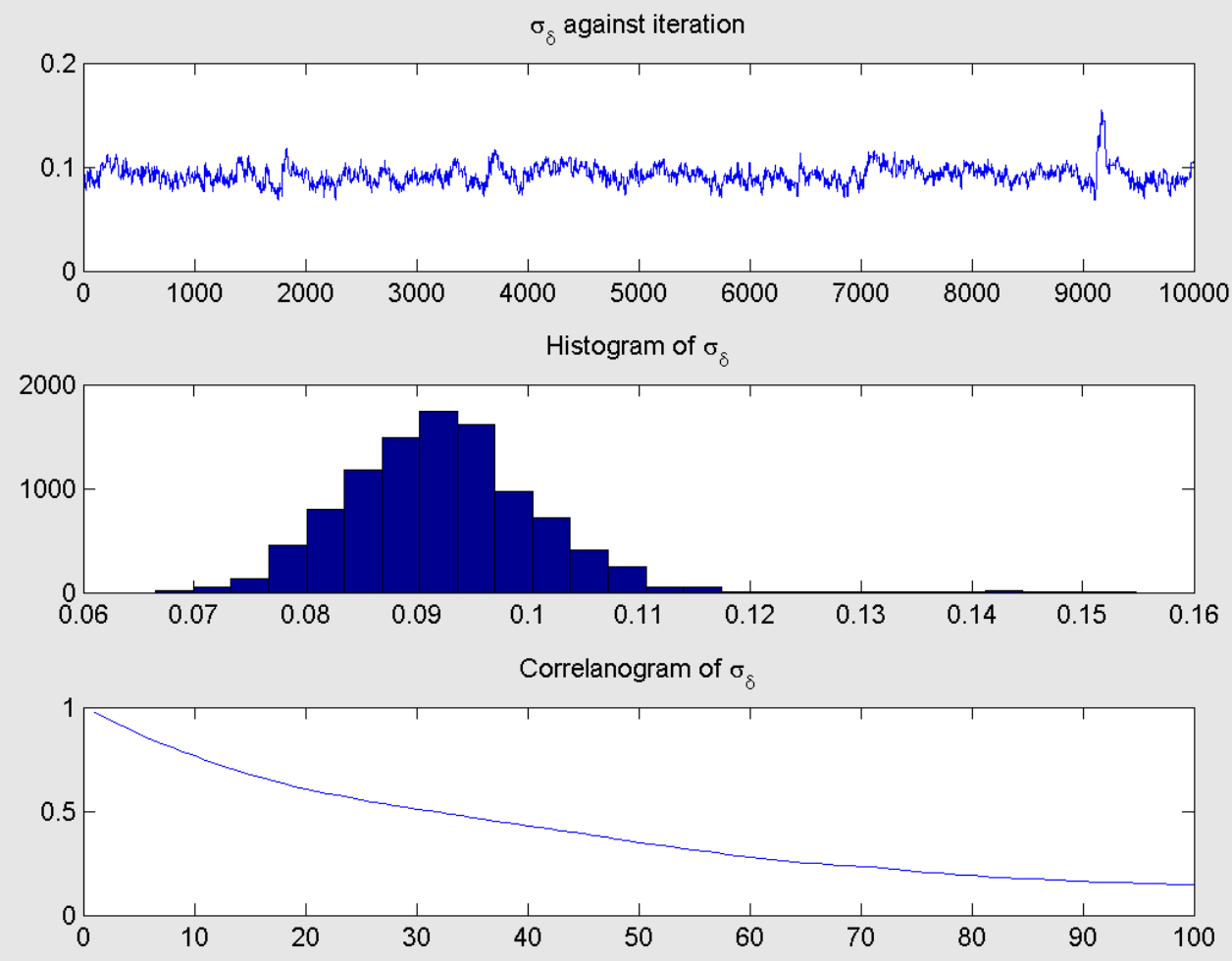

Figura 16: Simulação de $\sigma_{\delta} \mid \mathbf{y}$ utilizando o modelo CYES e dados de cobre O gráfico superior exibe a cadeia simulada para o parâmetro. O gráfico intermediario é o histograma dos valores simulados. O gráfico inferior é o correlograma da cadeia simulada. 

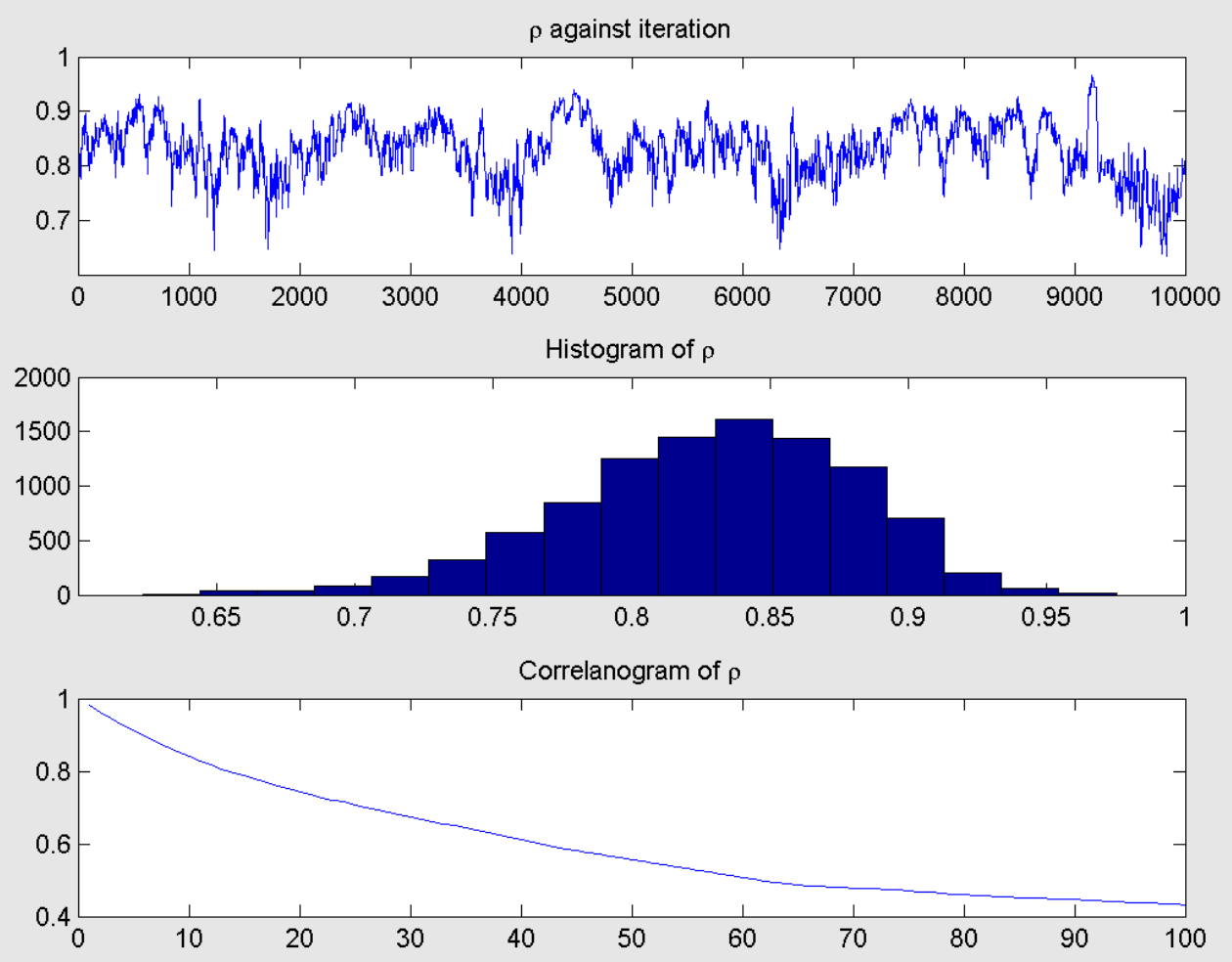

Figura 17: Simulação de $\rho \mid$ y utilizando o modelo CYES e dados de cobre O gráfico superior exibe a cadeia simulada para o parâmetro. O gráfico intermediario é o histograma dos valores simulados. O gráfico inferior é o correlograma da cadeia simulada.
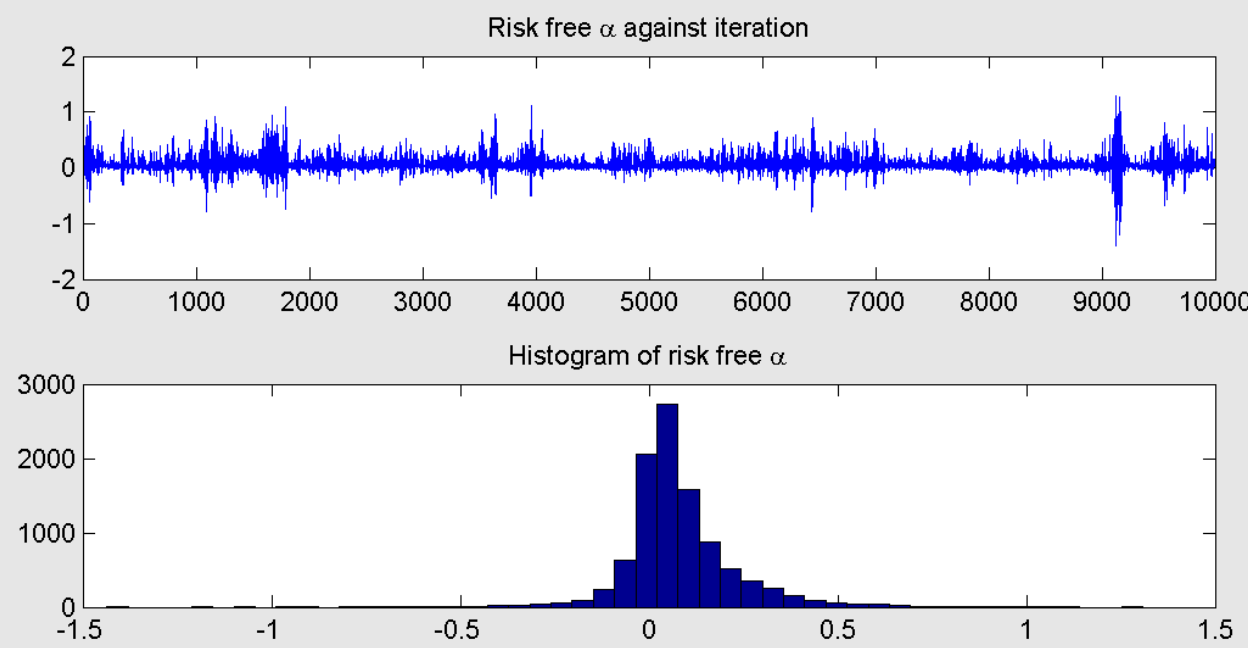

Correlanogram of risk free $\alpha$

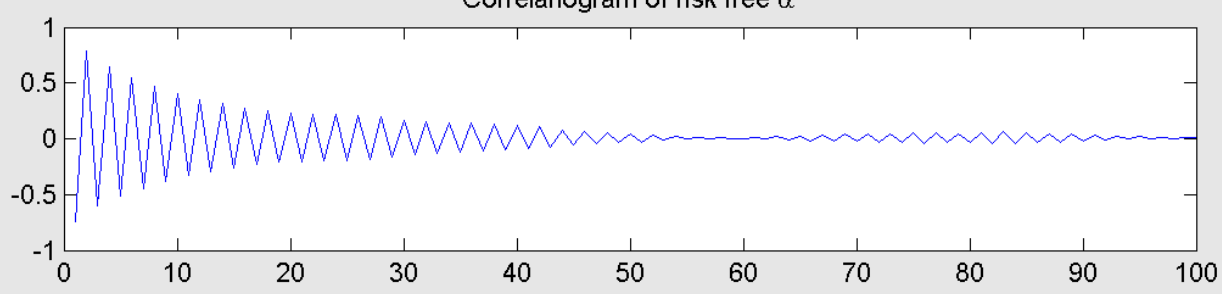

Figura 18: Simulação de $\hat{\alpha} \mid \mathbf{y}$ utilizando o modelo CYES e dados de cobre

O gráfico superior exibe a cadeia simulada para o parâmetro. O gráfico intermediario é o histograma dos valores simulados. O gráfico inferior é o correlograma da cadeia simulada. 

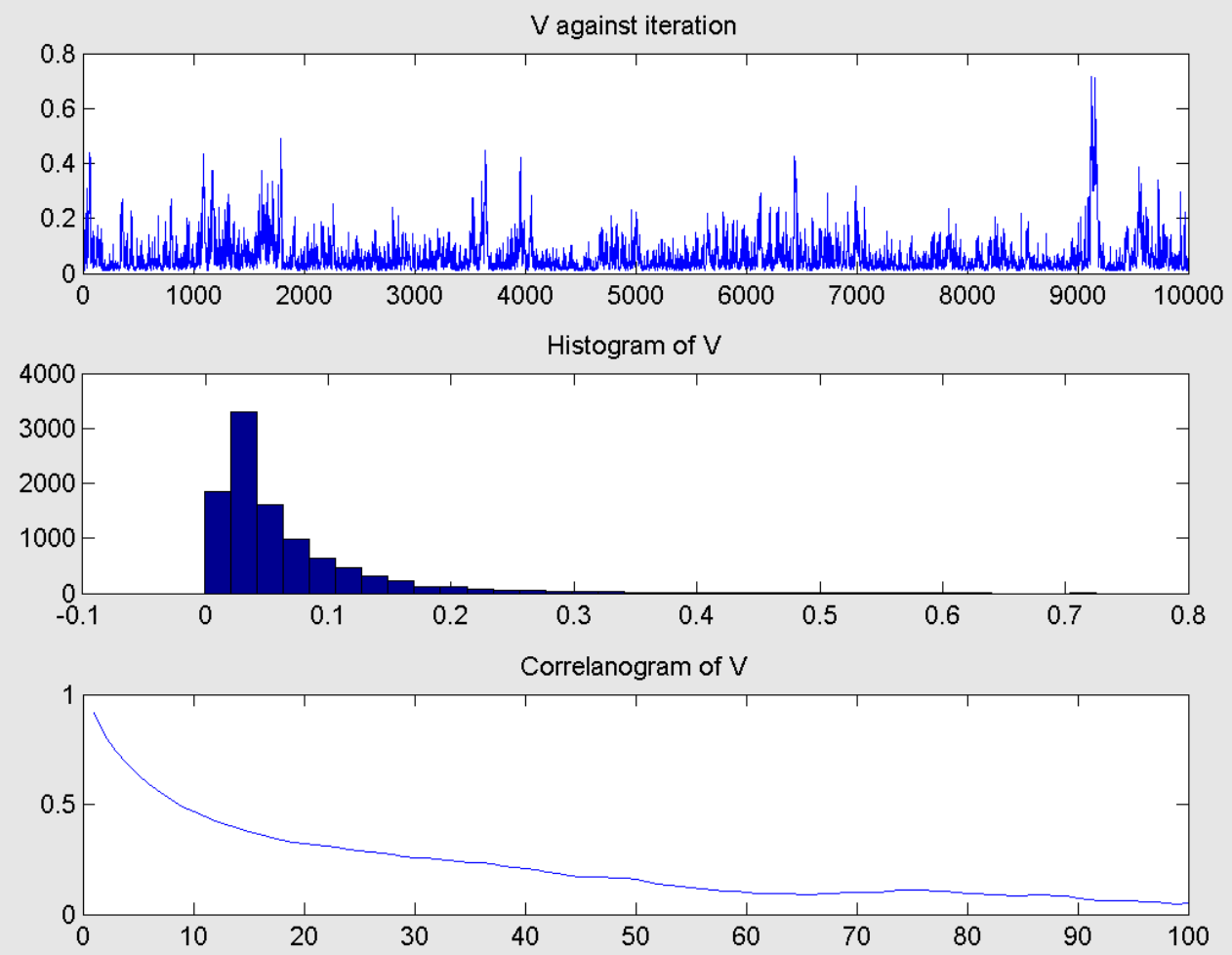

Figura 19: Simulação de $\boldsymbol{V} \mid \mathbf{y}$ utilizando o modelo CYES e dados de cobre O gráfico superior exibe a cadeia simulada para o parâmetro. O gráfico intermediario é o histograma dos valores simulados. O gráfico inferior é o correlograma da cadeia simulada.
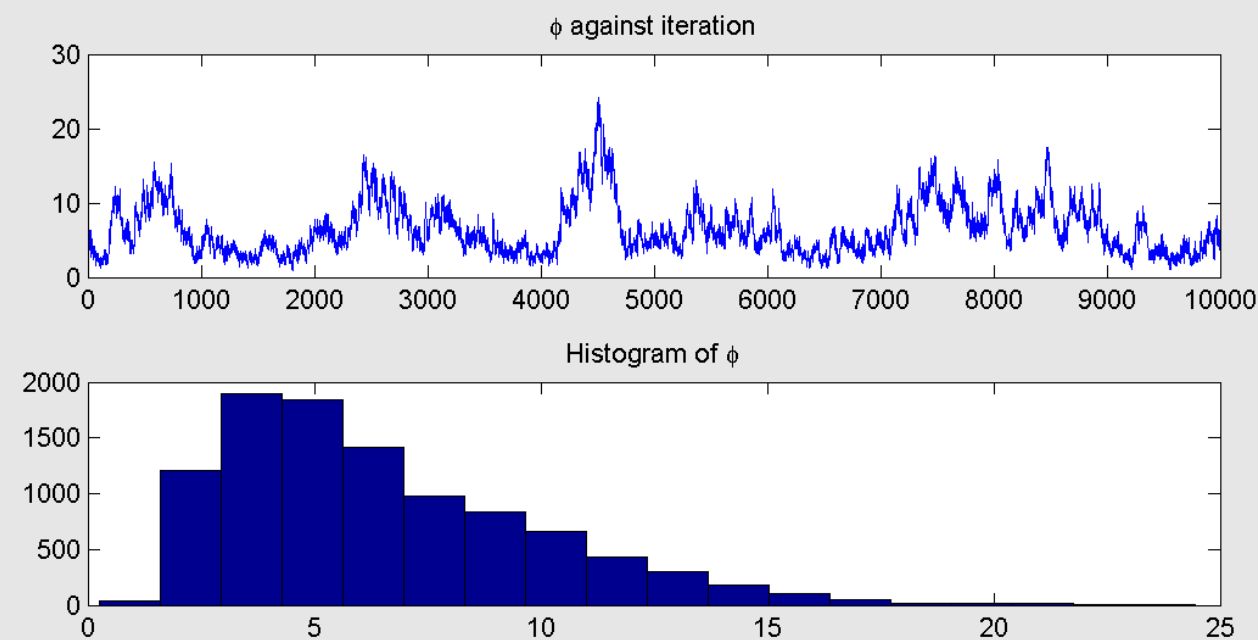

Correlanogram of $\phi$

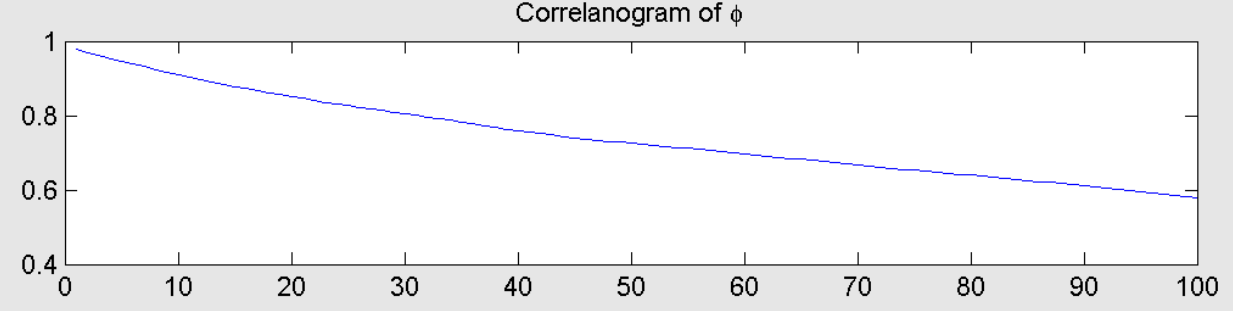

Figura 20: Simulação de $\phi \mid$ y utilizando o modelo CYES e dados de cobre O gráfico superior exibe a cadeia simulada para o parâmetro. O gráfico intermediario é o histograma dos valores simulados. O gráfico inferior é o correlograma da cadeia simulada. 

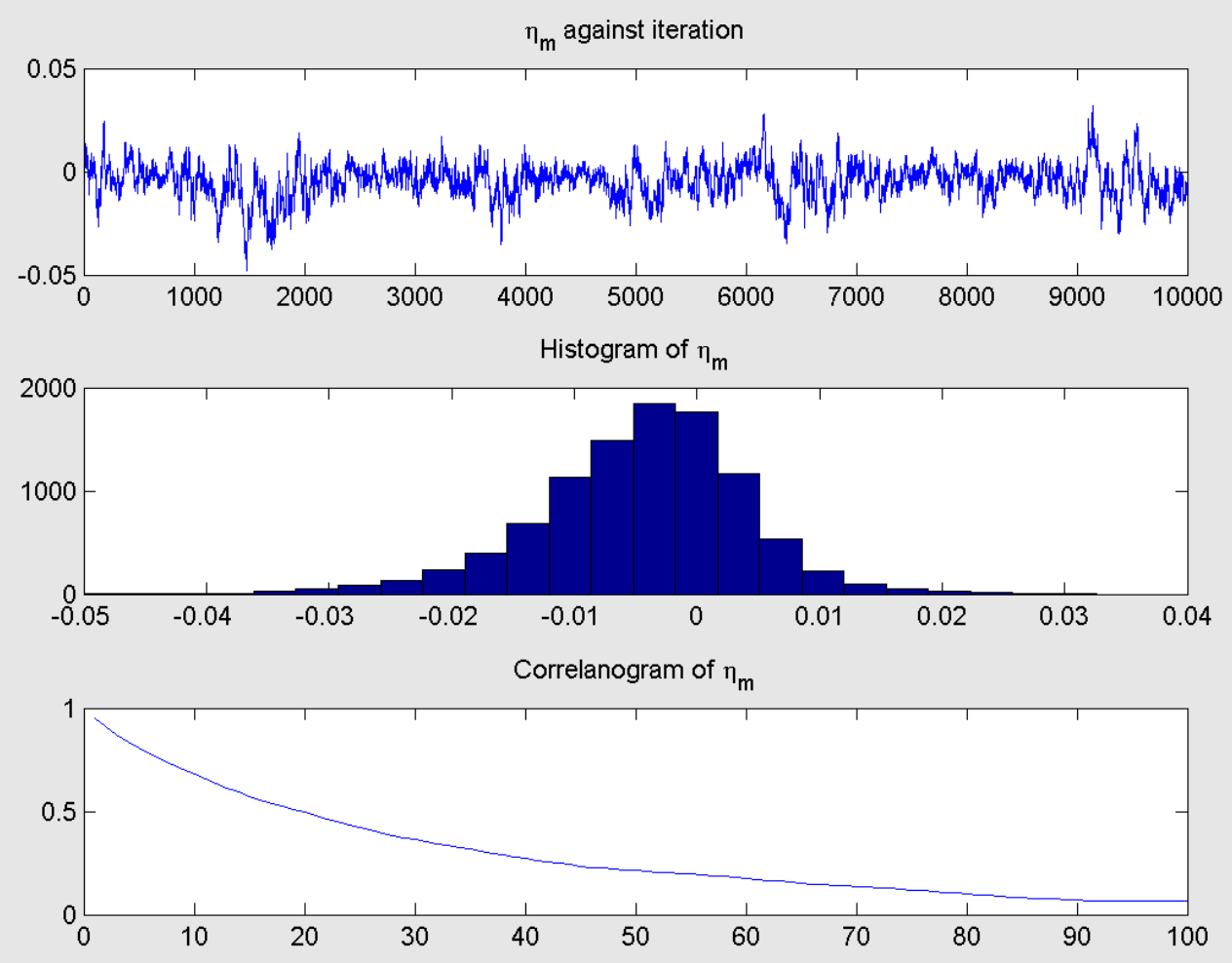

Figura 21: Simulação de $\overline{\boldsymbol{\eta}}_{m} \mid$ y utilizando o modelo CYES e dados de cobre

O gráfico superior exibe a cadeia simulada para o parâmetro. O gráfico intermediario é o histograma dos valores simulados. O gráfico inferior é o correlograma da cadeia simulada.
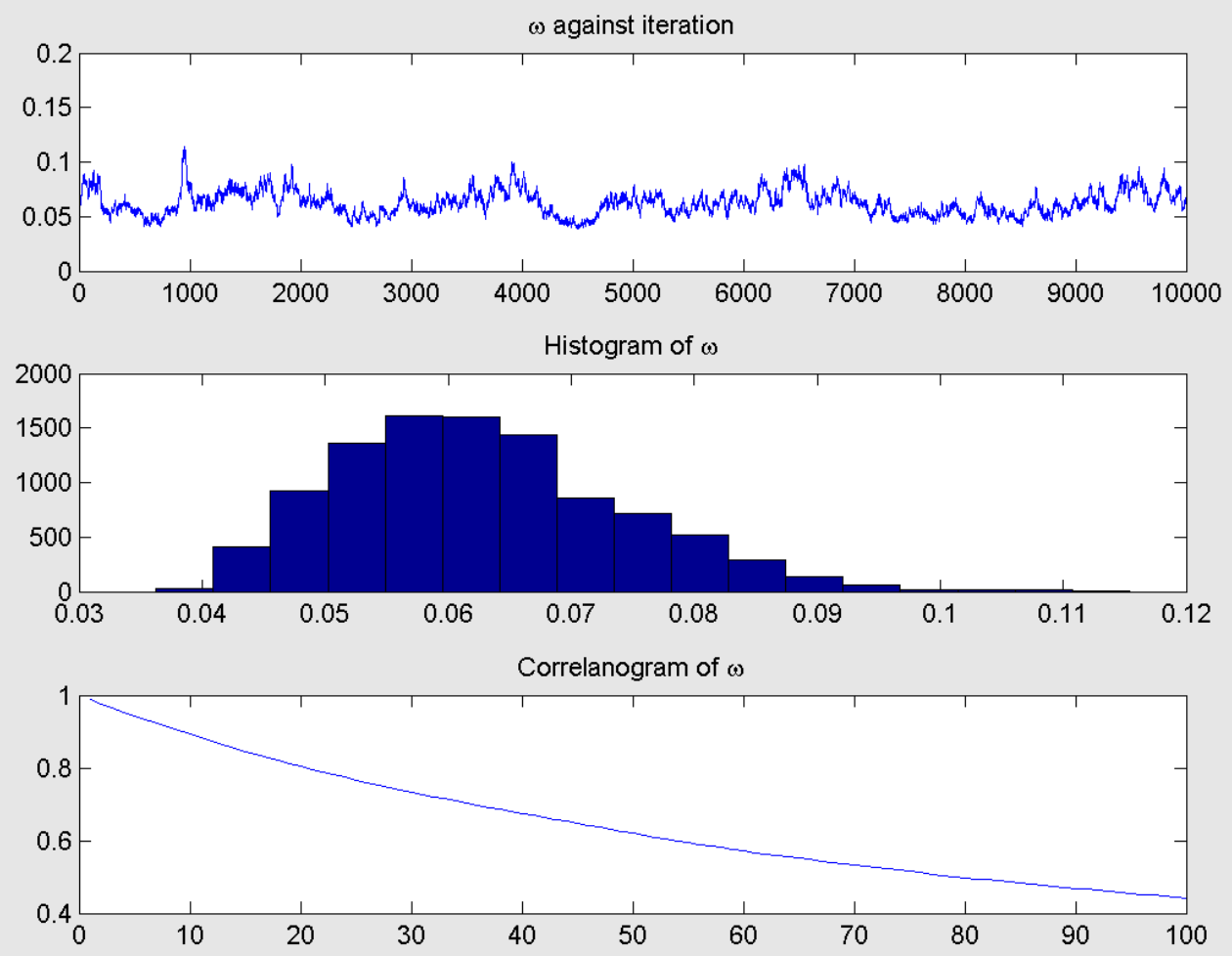

Figura 22: Simulação de $\boldsymbol{\omega} \mid \mathbf{y}$ utilizando o modelo CYES e dados de cobre

O gráfico superior exibe a cadeia simulada para o parâmetro. O gráfico intermediario é o histograma dos valores simulados. O gráfico inferior é o correlograma da cadeia simulada. 


\section{Bibliografia}

Bates, D. S. (1991). The crash of '87: Was it expected? the evidence from options markets. The Journal of Finance, 46(3), 1009-1044.

Bjerksund, P. (1991). Contingent claims evaluation when the convenience yield is stochastic: Analytic results. Working Paper, Nonvegian School of Economics and Business Administration, 1-16.

Bjorn Eraker, M. J. \& Polsonn, N. (2003). The impact of jumps in volatility and returns. The Journal of Finance, 58(3), 1269-1300.

Black, F. (1976). The pricing of commodity contracts. Journal of Financial Economics, 5(2), 167-179.

Chib, S. (2001). Markov chain monte carlo methods: Computation and inference. Handbook of Econometrics, 5, 3569-3649.

Gibson, R. \& Schwartz, E. S. (1990). Stochastic convenience yield and the pricing of oil contingent claims. The Journal of Finance, 45 (3), 959-976.

Gurdip Bakashi, C. C. \& Chen, Z. (1997). Empirical performance of alternative option pricing models. The Journal of Finance, 102(3), 2003-2049.

Hilliard, J. E. \& Reis, J. (1998). The jump-risk premia implicit in options: Evidence from an integrated time-series study. The Journal of Financial and Quantitative Analysis, $33(1), 81-86$.

Honoré, P. (1998). Pitfalls in estimating jump-diffusion models. Centre for Analytical Finance Working Paper Series, (18), 1-28.

Johannes, M. \& Polson, N. (2002). Mcmc methods for continuous-time financial econometrics. Handbook of Financial Econometrics, 2, 1-72.

Jong, P. D. \& Shephard, N. (1995). The simulation smoother for time series models. Biometrika, 82(2), 339-350.

Longstaff, F. A. \& Schwartz, E. S. (2001). Valuing american options by simulation: A simple least-squares approach. Review of Financial Studies, 14(1), 113-147.

Merton, R. C. (1976). Option pricing when underlying stock returns are discontinuous. Journal of Financial Economics, 3(1), 125-144.

Pan, J. (2002). The jump-risk premia implicit in options: Evidence from an integrated time-series study. Journal of Financial Economics, 63(1), 3-50.

Schwartz, E. S. (1997). The stochastic behavior of commodity prices: Implications for valuation and hedging. The Journal of Finance, 52(3), 923-973.

Working, H. (1949). The theory of price of storage. The American Economic Review, 39 (6), 1254-1262. 